\title{
WAVE LENGTH MEASUREMENTS IN THE ARC SPEC- TRA OF NEODYMIUM AND SAMARIUM
}

\author{
By C. C. Kiess
}

\begin{abstract}
The arc spectra of neodymium and samarium were photographed from $5475 \mathrm{~A}$ in the green out into the infra-red. The materials used for the work were neodymium oxalate and samarium oxide prepared at the University of Illinois and samarium oxalate prepared at New Hampshire College. The wave length tables contain about I500 lines for $\mathrm{Nd}$ and about the same number for Sa. A third table contains 130 lines which were common to both spectra. These lines are of unknown origin and may belong to the missing element of order number $6 \mathrm{r}$, coming between Nd and Sa. The methods used for the work are identical with those described in a previous work on yttrium, lanthanum, and cerium.
\end{abstract}

\section{CONTENTS}

Page,

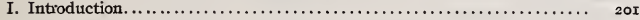

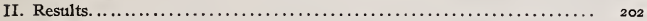

r. Neodymium.................................. 203

2. Samarium. . . . . . . . . . .

3. Lines of unknown origin. .......................

\section{INTRODUCTION}

In this paper are presented tables of wave lengths in the are spectra of neodymium and samarium, extending from the yellow into the infra-red regions. This work continues an investigation of the spectra of the rare earth elements which was undertaken at the Bureau of Standards several years ago. A previous paper ${ }^{1}$ contains results for the three elements, yttrium, lanthanum, and cerium, and describes the methods used for securing the spectrograms and deriving the final data from them. Inasmuch as the observational work on the various members of the rare earth group of elements was done at the same time, the reader is referred to this earlier paper for various details describing the work. The discussion in the present paper will therefore be limited to a statement of the results obtained for the elements neodymium and samarium.

I B. S. Sei. Papers, 17, p. 318; 1921. 


\section{RESULTS}

The chief characteristic of the spectra tabulated below is the enormous numbers of lines, mostly faint, which they contain. These numbers are limited, apparently, only by the duration of the exposures and the sensitivity of the photographic plates. The tables are arranged (as in previous papers ${ }^{2}$ ) so as to exhibit in column $\mathrm{I}$, the wave length in international units; in column 2 , the intensity and character of the lines; and in column 3 , the probable error.

The second and third columns of the tables give symbols describing the intensity, character, and accuracy of the lines. The intensities range from I for the faintest lines measured to Io for the strongest. The letters and symbols in the explanatory columns have the following significance:

$\mathrm{b}=$ broad.

coin $=$ coincident.

$\mathrm{d}=$ double.

$\mathrm{g}=$ ghost.

$\mathrm{h}=$ hazy.

$1=$ shaded to red.

$\mathrm{n}=$ band head.

$\mathrm{p}=$ part of band structure.
$\mathrm{R}=$ reversed.

$\mathrm{v}=$ shaded to violet.

$\mathrm{A}=$ probable error of $0.000 \mathrm{~A}$ to $0.010 \mathrm{~A}$.

$\mathrm{B}=$ probable error of $0.010 \mathrm{~A}$ to $0.020 \mathrm{~A}$.

$\mathrm{C}=$ probable error of $0.020 \mathrm{~A}$ to $0.030 \mathrm{~A}$.

$\mathrm{D}=$ probable error $>0.030 \mathrm{~A}$.

$\mathrm{E}=$ only one determination.

When the tabulated wave lengths for neodymium and samarium were intercompared, it was found that many lines, for the most part faint, were common to the spectra of both. It has not been possible to identify them with the spectra of known elements, and, therefore, they have been brought together in a separate table. While it would be a hasty conclusion to regard these lines as characteristic of a new element, yet the idea seems plausible in view of the fact that the present accepted scheme of the periodic table of elements requires a new element of atomic number $6 \mathrm{I}$ coming between neodymium and samarium. ${ }^{3}$ Eder ${ }^{4}$ has published similar lists of unidentified lines which he suggests may belong to elements not yet isolated, but none of the lines of Table 3 below agree with those in his lists.

\section{NEODYMIUM}

Table $\mathrm{x}$ contains about $\mathrm{I} 500$ lines measured between the limits $5474 \mathrm{~A}$ and $8936 \mathrm{~A}$ in the arc spectrum of neodymium. Most of these lines are faint, very few being of intensity 4 or greater. A

${ }^{2}$ B. S. Sci. Papers, 17, p. 321; 1921.

3 Sommerfeld, Atombau und Spektrallinien, pp. 55 and 58 , ist ed.: 1919.

${ }^{4}$ Kaiserl. Akad. der Wiss. Sitz. Wien, 126, Ila, D. 523; r917. Akad, der Wiss. Sitz, Wien, 129, IIa, p. 434; 1920. 
banded spectrum is practically absent, there being only a few faint lines that somewhat resemble band heads.

The material which was used for the majority of the spectrograms was neodymium oxalate prepared at the University of Illinois under the direction of Prof. B. S. Hopkins and kindly supplied by him to the Bureau of Standards for spectroscopic analysis. $\mathrm{He}$ has described the preparation of this material in a previous paper. ${ }^{5}$ Comparison of the spectrum of this material with the spectra of other elements indicates that, with the exception noted above, it is very pure. In column 2 of the table agreements of several wave lengths with those of other elements are indicated, but it is believed that these are coincidences only, without any significance. In addition to the neodymium oxalate there were available for analysis a sample of magnesium neodymium nitrate and a sample of "didymium," each of unknown origin. Neither was very pure, being contaminated with other elements of the rare earth group; but the values of neodymium lines observed with them have been included in the means given below.

Eder and Valenta ${ }^{6}$ have measured the spectrum of neodymium from $5473 \mathrm{~A}$ to $7808 \mathrm{~A}$, using a neodymium chloride prepared by C. Auer von Welsbach. Their results are given on the Rowland scale. A later work by Eder, ${ }^{7}$ using neodymium sulphate, also prepared by Auer, gives the spectrum in international units from $6432 \mathrm{~A}$ to $7808 \mathrm{~A}$. The measurements of these investigators and those tabulated below are in good accord in the regions common to both.

TABLE 1.-Arc Spectrum of Neodymium

\begin{tabular}{|c|c|c|c|c|c|c|c|c|}
\hline$\lambda$ I. A. & Notes & $\begin{array}{c}\text { Probable } \\
\text { error }\end{array}$ & $\lambda$ I. A. & Notes & $\begin{array}{c}\text { Probable } \\
\text { error }\end{array}$ & $\lambda$ I. A. & Notes & $\begin{array}{c}\text { Probable } \\
\text { error }\end{array}$ \\
\hline $\begin{array}{l}5474.72 \\
5475.41 \\
5476.64 \\
5477.36 \\
5478.70\end{array}$ & $\begin{array}{l}2 \\
1 \\
1 \\
1 \\
1 \mathrm{~b}, 1\end{array}$ & $\begin{array}{l}\mathbf{B} \\
\mathbf{B} \\
\mathbf{B} \\
\mathbf{C} \\
\mathrm{C}\end{array}$ & $\begin{array}{l}5500.06 \\
5500.65 \\
5501.50 \\
5502.60 \\
5503.72\end{array}$ & $\begin{array}{l}1 \\
1 \\
3 \\
1 \\
1\end{array}$ & $\begin{array}{l}\mathbf{B} \\
\mathbf{B} \\
\mathbf{B} \\
\mathbf{B} \\
\mathbf{B}\end{array}$ & $\begin{array}{l}5512.61 \\
5514.00 \\
5514.75 \\
5515.64 \\
5516.00\end{array}$ & $\begin{array}{l}1 \\
1 \\
1 \\
1 \\
1\end{array}$ & $\begin{array}{l}\mathbf{B} \\
\mathbf{E} \\
\mathbf{C} \\
\mathbf{B} \\
\mathbf{C}\end{array}$ \\
\hline $\begin{array}{l}5483.95 \\
5485.04 \\
5485.72 \\
5487.02 \\
5492.28\end{array}$ & $\begin{array}{l}1 \\
3 \\
5 \\
2 \\
2\end{array}$ & $\begin{array}{l}\text { C } \\
\text { B } \\
B \\
B \\
B\end{array}$ & $\begin{array}{l}5504.52 \\
5504.98 \\
5505.34 \\
5505.85 \\
5506.71\end{array}$ & $\begin{array}{l}1 \\
1 \\
1 \\
1 \\
1\end{array}$ & $\begin{array}{l}\mathbf{E} \\
\mathbf{E} \\
\mathrm{E} \\
\mathbf{E} \\
\mathbf{E}\end{array}$ & $\begin{array}{l}5516.29 \\
5516.90 \\
5518.40 \\
5518.74 \\
5519.42\end{array}$ & $\begin{array}{ll}2 & \\
1 & \\
1 & \mathrm{ES} \\
2 & \\
2 & 1\end{array}$ & $\begin{array}{l}\text { A } \\
\mathbf{C} \\
\mathbf{B} \\
\mathbf{B} \\
\mathbf{B}\end{array}$ \\
\hline $\begin{array}{l}5493.37 \\
5494.01 \\
5495.56 \\
5496.42 \\
5498.86\end{array}$ & $\begin{array}{l}2 \\
4 \\
1 \\
1 \\
1\end{array}$ & $\begin{array}{l}\mathbf{B} \\
\mathbf{B} \\
\mathbf{B} \\
\mathbf{B} \\
\mathbf{B}\end{array}$ & $\begin{array}{l}5507.19 \\
5507.63 \\
5509.12 \\
5510.70 \\
5511.93\end{array}$ & $\begin{array}{l}1 \\
2 \\
2 \\
1 \\
1\end{array}$ & $\begin{array}{l}\text { B } \\
\text { B } \\
\text { A } \\
\text { C } \\
\text { E }\end{array}$ & $\begin{array}{l}5520.02 \\
5520.66 \\
5522.16 \\
5522.89 \\
5525.74\end{array}$ & $\begin{array}{l}2 \\
3 \\
2 \\
1 \\
3\end{array}$ & $\begin{array}{l}\mathbf{B} \\
\mathbf{B} \\
\mathbf{B} \\
\mathbf{E} \\
\mathbf{A}\end{array}$ \\
\hline
\end{tabular}

5 B. S. Sci. Papers, 17, p 342; I92 I.

Kaiserl. Akad, der Wiss. Sitz. Wien, 119, IIa, D. 554; I910.

7 Kaiserl. Akad. der Wiss, Sitz. Wien, 124, I1a, p. 110; I915. 
TABLE 1.-Arc Spectrum of Neodymium-Continued

\begin{tabular}{|c|c|c|c|c|c|c|c|c|}
\hline$\lambda$ I. A. & Notes & $\begin{array}{c}\begin{array}{c}\text { Probable } \\
\text { error }\end{array} \\
\text {. }\end{array}$ & $\lambda$ I. A. & Notes & $\begin{array}{c}\text { Probable } \\
\text { error }\end{array}$ & $\lambda$ I. A. & Notes & $\begin{array}{c}\text { Probable } \\
\text { error }\end{array}$ \\
\hline $\begin{array}{l}5526.13 \\
5526.68 \\
5527.01 \\
5528.36 \\
5529.05\end{array}$ & $\begin{array}{l}1 \\
1 \\
1 \\
3 \\
2 \text { ES }\end{array}$ & $\begin{array}{l}\mathbf{B} \\
\mathbf{E} \\
\mathbf{B} \\
\mathbf{B} \\
\mathbf{A}\end{array}$ & $\begin{array}{l}5577.75 \\
5578.63 \\
5579.49 \\
5579.93 \\
5580.49\end{array}$ & $\begin{array}{l}3 \\
3 \\
1 \\
1 \\
1\end{array}$ & $\begin{array}{l}\mathbf{B} \\
\mathbf{B} \\
\mathbf{E} \\
\mathbf{B} \\
\mathbf{B}\end{array}$ & $\begin{array}{l}5634.27 \\
5634.66 \\
5635.74 \\
5636.22 \\
5636.85\end{array}$ & $\begin{array}{l}1 \text { ES } \\
1 \\
3 \\
1 \\
1\end{array}$ & $\begin{array}{l}\mathbf{E} \\
\mathbf{E} \\
\mathbf{A} \\
\mathbf{E} \\
\mathbf{B}\end{array}$ \\
\hline $\begin{array}{l}5529.36 \\
5529.75 \\
5530.48 \\
5532.08 \\
5532.44\end{array}$ & $\begin{array}{l}1 \\
1 \\
2 \\
2 \\
1\end{array}$ & $\begin{array}{l}\mathbf{E} \\
\mathrm{E} \\
\mathbf{B} \\
\mathbf{B} \\
\mathbf{C}\end{array}$ & $\begin{array}{l}5581.59 \\
5582.03 \\
5582.36 \\
5582.80 \\
5583.62\end{array}$ & $\begin{array}{l}3 \\
1 \\
2 \\
1 \\
1\end{array}$ & $\begin{array}{l}\mathbf{A} \\
\mathbf{E} \\
\mathbf{A} \\
\mathbf{E} \\
\mathbf{E}\end{array}$ & $\begin{array}{l}5637.20 \\
5637.77 \\
5639.00 \\
5639.54 \\
5640.50\end{array}$ & $\begin{array}{l}1 \\
2 \mathrm{~b} \\
2 \mathrm{v} \\
3 \\
2\end{array}$ & $\begin{array}{l}\mathbf{E} \\
\mathbf{B} \\
\mathbf{B} \\
\mathbf{B} \\
\mathbf{B}\end{array}$ \\
\hline $\begin{array}{l}5532.94 \\
5533.40 \\
5533.82 \\
5535.27 \\
5536.37\end{array}$ & $\begin{array}{l}2 \\
2 \\
4 \\
3 \\
2 v\end{array}$ & $\begin{array}{l}\mathbf{A} \\
\mathbf{A} \\
\mathbf{A} \\
\mathbf{A} \\
\mathbf{B}\end{array}$ & $\begin{array}{l}5584.20 \\
5584.41 \\
\mathbf{5 5 8 6 . 0 6} \\
5587.59 \\
5587.95\end{array}$ & $\begin{array}{l}2 \\
1 \\
1 \\
\frac{1}{2} \\
3\end{array}$ & $\begin{array}{l}\mathrm{B} \\
\mathrm{E} \\
\mathrm{E} \\
\mathrm{A} \\
\mathrm{A}\end{array}$ & $\begin{array}{l}\mathbf{5 6 4 2 .} .08 \\
5642.64 \\
5643.24 \\
5644.14 \\
\mathbf{5 6 4 5 . 1 8}\end{array}$ & $\begin{array}{l}1 \text { ES } \\
1 \\
2 \\
2 \\
1\end{array}$ & $\begin{array}{l}\mathrm{E} \\
\mathbf{B} \\
\mathrm{B} \\
\mathrm{B} \\
\mathrm{E}\end{array}$ \\
\hline $\begin{array}{l}5536.85 \\
5537.29 \\
5537.76 \\
5538.79 \\
5540.42\end{array}$ & $\begin{array}{l}1 \\
2 \\
2 \\
1 \\
1\end{array}$ & $\begin{array}{l}\mathbf{E} \\
\mathrm{A} \\
\mathrm{A} \\
\mathrm{A} \\
\mathrm{E}\end{array}$ & $\begin{array}{l}5588.39 \\
5588.84 \\
5589.94 \\
5590.67 \\
5592.65\end{array}$ & $\begin{array}{l}1 \\
3 \\
1 \\
2 \mathrm{~b} \\
3\end{array}$ & $\begin{array}{l}\mathbf{E} \\
\text { A } \\
\text { E } \\
\text { C } \\
\text { A }\end{array}$ & $\begin{array}{l}5645.50 \\
5647.34 \\
5647.98 \\
5648.37 \\
56-49.22\end{array}$ & $\begin{array}{l}1 \\
1 \\
2 \\
1 \\
1\end{array}$ & $\begin{array}{l}\mathrm{E} \\
\mathrm{E} \\
\mathrm{B} \\
\mathrm{E} \\
\mathrm{B}\end{array}$ \\
\hline $\begin{array}{l}5540.77 \\
5541.76 \\
5543.24 \\
5543.88 \\
5544.30\end{array}$ & $\begin{array}{l}1 \\
1 \\
3 \\
1 \mathrm{~b} \\
1\end{array}$ & $\begin{array}{l}\mathrm{B} \\
\mathrm{E} \\
\mathrm{A} \\
\mathrm{B} \\
\mathrm{E}\end{array}$ & $\begin{array}{l}5594.43 \\
5594.97 \\
5595.83 \\
5596.61 \\
5597.81\end{array}$ & $\begin{array}{l}6 \mathrm{Ca} ? \\
1 \\
31 \\
1 \\
2 \mathrm{~V}\end{array}$ & $\begin{array}{l}\text { A } \\
\text { B } \\
\text { A } \\
\text { E } \\
\text { B }\end{array}$ & $\begin{array}{l}5649.80 \\
5651.23 \\
5652.67 \\
5653.55 \\
5654.67\end{array}$ & $\begin{array}{l}1 \\
2 \\
1 \\
3 \mathrm{v}, \mathrm{d} ? \\
1\end{array}$ & $\begin{array}{l}\mathrm{E} \\
\mathrm{B} \\
\mathrm{B} \\
\mathrm{B} \\
\mathrm{B}\end{array}$ \\
\hline $\begin{array}{l}5544.87 \\
5545.90 \\
5546.10 \\
5546.60 \\
5546.96\end{array}$ & $\begin{array}{l}1 \mathrm{~b}, 1 \\
3 \\
1 \\
1 \\
2\end{array}$ & $\begin{array}{l}\mathbf{E} \\
\mathrm{A} \\
\mathrm{E} \\
\mathrm{B} \\
\mathbf{B}\end{array}$ & $\begin{array}{l}5598.50 \\
5598.84 \\
5599.99 \\
5601.41 \\
5601.91\end{array}$ & $\begin{array}{l}2 \mathrm{Ca} ? \\
1 \mathrm{~b} \\
\frac{1}{3} \mathrm{~b} \\
3\end{array}$ & $\begin{array}{l}\text { B } \\
\text { B } \\
\text { B } \\
\text { A } \\
\text { A }\end{array}$ & $\begin{array}{l}\mathbf{5 6 5 5 . 6 3} \\
5656.49 \\
5656.90 \\
5657.37 \\
\mathbf{5 6 5 8 . 3 6}\end{array}$ & $\begin{array}{l}1 \\
2 \\
1 \\
1 \\
2\end{array}$ & $\begin{array}{l}\mathrm{C} \\
\mathbf{A} \\
\mathbf{B} \\
\mathbf{B} \\
\mathbf{B}\end{array}$ \\
\hline $\begin{array}{l}5547.72 \\
5548.45 \\
5548.68 \\
5550.09 \\
5550.75\end{array}$ & $\begin{array}{l}2 \\
4 \\
3 \\
3 \\
1\end{array}$ & $\begin{array}{l}\text { C } \\
\text { A } \\
\text { A } \\
\text { A } \\
\text { E }\end{array}$ & $\begin{array}{l}5602.63 \\
5604.28 \\
5605.62 \\
5606.15 \\
5606.63\end{array}$ & $\begin{array}{l}4 \\
1 \\
3 \mathrm{~b}, \operatorname{Pr} ? \\
1 \\
1\end{array}$ & $\begin{array}{l}\mathbf{B} \\
\mathbf{D} \\
\mathbf{B} \\
\mathbf{E} \\
\mathbf{E}\end{array}$ & $\begin{array}{l}5659.77 \\
5661.42 \\
5661.86 \\
5662.44 \\
5663.56\end{array}$ & $\begin{array}{l}4 \\
1 \\
1 \\
1 \\
2 \\
2\end{array}$ & $\begin{array}{l}\text { A } \\
\text { B } \\
\mathbf{E} \\
\text { B } \\
\mathbf{B}\end{array}$ \\
\hline $\begin{array}{l}5551.91 \\
5552.87 \\
5553.31 \\
5553.79 \\
5555.02\end{array}$ & $\begin{array}{l}1 \\
2 \\
1 \\
1 \mathrm{~b} \\
1\end{array}$ & $\begin{array}{l}\mathrm{C} \\
\mathrm{B} \\
\mathrm{E} \\
\mathrm{E} \\
\mathrm{E}\end{array}$ & $\begin{array}{l}5607.13 \\
5607.50 \\
5607.86 \\
5608.81 \\
5610.30\end{array}$ & $\begin{array}{l}1 \\
1 \\
1 \\
1 \\
1 \\
2\end{array}$ & $\begin{array}{l}\mathrm{E} \\
\mathrm{E} \\
\mathrm{E} \\
\mathrm{E} \\
\mathrm{E}\end{array}$ & $\begin{array}{l}5664.26 \\
5665.25 \\
5665.73 \\
5666.54 \\
5668.23\end{array}$ & $\begin{array}{l}1 \\
2 \\
2 \\
1 \\
1\end{array}$ & $\begin{array}{l}\text { B } \\
\text { B } \\
\text { B } \\
\text { B } \\
\text { E }\end{array}$ \\
\hline $\begin{array}{l}5555.73 \\
5556.64 \\
5558.98 \\
5559.81 \\
5561.16\end{array}$ & $\begin{array}{l}2 \\
1 \\
2 \\
2 \\
4\end{array}$ & $\begin{array}{l}\mathrm{A} \\
\mathrm{E} \\
\mathrm{B} \\
\mathrm{B} \\
\mathrm{A}\end{array}$ & $\begin{array}{l}5611.15 \\
5611.41 \\
5612.12 \\
5612.41 \\
5613.13\end{array}$ & $\begin{array}{l}2 \\
1 \\
1 \\
1 \\
1 \\
1\end{array}$ & $\begin{array}{l}\mathrm{B} \\
\mathrm{E} \\
\mathrm{B} \\
\mathrm{E} \\
\mathrm{E}\end{array}$ & $\begin{array}{l}5668.85 \\
5659.28 \\
5669.77 \\
5670.88 \\
5671.77\end{array}$ & $\begin{array}{l}4 \\
2 \\
3 \\
1 \\
1 \text { b, ES }\end{array}$ & $\begin{array}{l}\mathbf{A} \\
\mathbf{B} \\
\mathbf{A} \\
\mathbf{E} \\
\mathbf{C}\end{array}$ \\
\hline $\begin{array}{l}5562.12 \\
5563.27 \\
5564.53 \\
5565.15 \\
5565.76\end{array}$ & $\begin{array}{l}\frac{1}{b}, \operatorname{Pr} ? \\
1 \\
1 \\
1 \mathrm{~b} \\
2 \mathrm{~b}\end{array}$ & $\begin{array}{l}\mathrm{B} \\
\mathrm{E} \\
\mathrm{E} \\
\mathrm{B} \\
\mathrm{B}\end{array}$ & $\begin{array}{l}5613.49 \\
5614.26 \\
5614.70 \\
5615.29 \\
5616.16\end{array}$ & $\begin{array}{l}1 \\
4 \\
1 \\
3 \\
1\end{array}$ & $\begin{array}{l}\underset{\mathrm{B}}{\mathrm{E}} \\
\underset{\mathrm{B}}{\mathrm{B}} \\
\mathrm{E}\end{array}$ & $\begin{array}{l}5672.14 \\
5672.67 \\
5673.22 \\
5674.83 \\
5675.92\end{array}$ & $\begin{array}{l}1 \\
1 \\
1 \\
2 \\
6\end{array}$ & $\begin{array}{l}\mathrm{E} \\
\mathrm{B} \\
\mathrm{B} \\
\mathrm{B} \\
\mathrm{A}\end{array}$ \\
\hline $\begin{array}{l}5566.15 \\
5567.24 \\
5567.65 \\
5568.15 \\
5568.66\end{array}$ & $\begin{array}{l}1 \\
1 \\
1 \\
1 \\
1\end{array}$ & $\begin{array}{l}\mathbf{B} \\
\mathbf{B} \\
\mathbf{B} \\
\mathbf{E} \\
\mathbf{E}\end{array}$ & $\begin{array}{l}5616.58 \\
5617.16 \\
5619.03 \\
5619.28 \\
5619.66\end{array}$ & $\begin{array}{l}1 \\
1 \\
3 \\
1 \\
1\end{array}$ & $\begin{array}{l}\mathbf{E} \\
\mathbf{E} \\
\mathbf{B} \\
\mathbf{E} \\
\mathbf{E}\end{array}$ & $\begin{array}{l}5676.35 \\
5677.81 \\
5679.58 \\
5680.19 \\
5681.16\end{array}$ & $\begin{array}{l}3 \\
2 \\
2 \\
1 \\
3\end{array}$ & $\begin{array}{l}\mathbf{A} \\
\mathbf{A} \\
\mathbf{B} \\
\mathbf{B} \\
\mathbb{A}\end{array}$ \\
\hline $\begin{array}{l}5569.05 \\
5569.95 \\
5570.71 \\
5571.06 \\
5571.54\end{array}$ & $\begin{array}{l}1 \\
4 \\
21, \mathrm{~d} \\
1 \\
1\end{array}$ & $\begin{array}{l}\mathrm{E} \\
\mathrm{A} \\
\mathrm{B} \\
\mathrm{E} \\
\mathrm{E}\end{array}$ & $\begin{array}{l}5620.62 \\
5622.28 \\
5622.84 \\
5624.26 \\
5625.33\end{array}$ & $\begin{array}{l}6 \\
2 \\
1 \\
2 \\
2 \\
2\end{array}$ & $\begin{array}{l}\mathbf{A} \\
\mathbf{B} \\
\mathbf{E} \\
\mathbf{B} \\
\mathbf{B}\end{array}$ & $\begin{array}{l}5682.51 \\
5683.16 \\
5633.69 \\
5684.20 \\
5684.62\end{array}$ & $\begin{array}{l}1 \\
2 \\
1 \\
1 \\
11, \mathbf{n} ?\end{array}$ & $\begin{array}{l}\mathbf{E} \\
\mathbf{B} \\
\mathbf{E} \\
\mathbf{E} \\
\mathbf{B}\end{array}$ \\
\hline $\begin{array}{l}5571.81 \\
5572.15 \\
5572.38 \\
5572.82 \\
5573.46\end{array}$ & $\begin{array}{l}1 \\
1 \\
1 \\
1 \\
1\end{array}$ & $\begin{array}{l}\mathrm{E} \\
\mathrm{E} \\
\mathrm{B} \\
\mathrm{E} \\
\mathrm{E}\end{array}$ & $\begin{array}{l}5625.71 \\
5626.64 \\
5627.16 \\
5628.41 \\
5628.94\end{array}$ & $\begin{array}{l}3 \\
2 \\
2 \mathrm{~b} \\
1 \\
1\end{array}$ & $\begin{array}{l}\mathrm{B} \\
\mathrm{B} \\
\mathrm{B} \\
\mathrm{D} \\
\mathrm{E}\end{array}$ & $\begin{array}{l}5585.00 \\
5085.45 \\
5686.60 \\
5687.16 \\
5687.84\end{array}$ & $\begin{array}{l}1 \\
1 \\
31 \\
1 \\
1\end{array}$ & $\begin{array}{l}\mathrm{E} \\
\mathrm{E} \\
\mathrm{B} \\
\mathrm{C} \\
\mathrm{B}\end{array}$ \\
\hline $\begin{array}{l}5574.03 \\
5574.66 \\
5574.98 \\
5575.50 \\
5576.70\end{array}$ & $\begin{array}{l}1 \\
2 \\
1 \\
3 \\
2\end{array}$ & $\begin{array}{l}\mathbf{E} \\
\mathbf{A} \\
\mathbf{E} \\
\mathbf{A} \\
\mathbf{A}\end{array}$ & $\begin{array}{l}5629.45 \\
5631.14 \\
5632.15 \\
5633.40 \\
5633.92\end{array}$ & $\begin{array}{l}1 \\
1 \\
1 \\
1 \\
1\end{array}$ & $\begin{array}{l}\mathrm{E} \\
\mathrm{E} \\
\mathrm{E} \\
\mathrm{E} \\
\mathrm{E}\end{array}$ & $\begin{array}{l}5688.50 \\
5689.06 \\
5689.50 \\
5690.20 \\
5691.06\end{array}$ & $\begin{array}{l}6 \\
1 \\
3 \\
2 \\
1\end{array}$ & $\begin{array}{l}\mathrm{A} \\
\mathrm{E} \\
\mathrm{B} \\
\mathrm{B} \\
\mathrm{B} \\
\mathrm{B}\end{array}$ \\
\hline
\end{tabular}


TABLE 1.-Arc Spectrum of Neodymium-Continued

\begin{tabular}{|c|c|c|c|c|c|c|c|c|}
\hline$\lambda$ I. A. & Notes & $\begin{array}{c}\text { Probable } \\
\text { error }\end{array}$ & $\lambda$ I. A. & Notes & $\begin{array}{c}\text { Probable } \\
\text { error }\end{array}$ & $\lambda$ I. A. & Notes & $\begin{array}{l}\text { Probable } \\
\text { error }\end{array}$ \\
\hline $\begin{array}{l}5691.72 \\
5693.03 \\
5693.88 \\
5695.18 \\
5695.65\end{array}$ & $\begin{array}{l}1 \text { b } \\
1 \text { b } \\
1 \\
2 \\
1\end{array}$ & $\begin{array}{l}\mathbf{B} \\
\mathbf{E} \\
\mathbf{E} \\
\mathbf{B} \\
\mathbf{B}\end{array}$ & $\begin{array}{l}5761.67 \\
5762.06 \\
5762.36 \\
5762.97 \\
5763.52\end{array}$ & $\begin{array}{l}3 \\
2 \\
2 \\
1 \\
1\end{array}$ & $\begin{array}{l}\text { B } \\
\text { B } \\
\text { B } \\
\text { E } \\
\text { E }\end{array}$ & $\begin{array}{l}5835.20 \\
5835.89 \\
5836.58 \\
5837.15 \\
5839.08\end{array}$ & $\begin{array}{l}2 \\
1 \\
1 \\
2 \\
2\end{array}$ & $\begin{array}{l}\mathbf{B} \\
\mathbf{E} \\
\mathbf{E} \\
\mathbf{A} \\
\mathbf{A}\end{array}$ \\
\hline $\begin{array}{l}5696.01 \\
5696.43 \\
5697.22 \\
5699.71 \\
5701.54\end{array}$ & $\begin{array}{l}1 \\
1 \\
2 \mathbf{b}, \mathbf{d} \text { ? } \\
\frac{1}{2}\end{array}$ & $\begin{array}{l}\mathbf{E} \\
\mathbf{E} \\
\mathbf{B} \\
\mathbf{E} \\
\mathbf{B}\end{array}$ & $\begin{array}{l}5764.20 \\
5766.53 \\
5767.33 \\
5768.41 \\
5769.86\end{array}$ & $\begin{array}{l}3 \\
2 \\
3 \\
1 \\
3\end{array}$ & $\begin{array}{l}\mathbf{A} \\
\text { A } \\
\text { A } \\
\mathbf{E} \\
\mathbf{B}\end{array}$ & $\begin{array}{l}5839.72 \\
5842.37 \\
5843.23 \\
5844.66 \\
5846.32\end{array}$ & $\begin{array}{l}2 \\
4 \\
2 \\
3 \\
2 \\
2\end{array}$ & $\begin{array}{l}\mathbf{A} \\
\mathbf{A} \\
\mathbf{A} \\
\mathbf{A} \\
\mathbf{A}\end{array}$ \\
\hline $\begin{array}{l}5702.25 \\
5704.41 \\
5705.08 \\
5707.33 \\
5708.28\end{array}$ & $\begin{array}{l}4 \\
1 \\
1 \\
2 \\
6\end{array}$ & $\begin{array}{l}\mathbf{A} \\
\mathbf{B} \\
\mathbf{E} \\
\mathbf{B} \\
\mathbf{A}\end{array}$ & $\begin{array}{l}5770.50 \\
5771.42 \\
5772.14 \\
5773.89 \\
5774.53\end{array}$ & $\begin{array}{l}3 \\
2 \\
2 \\
1 \\
2 \mathrm{~b}\end{array}$ & $\begin{array}{l}\mathbf{A} \\
\mathrm{E} \\
\mathrm{B} \\
\mathbf{E} \\
\mathrm{B}\end{array}$ & $\begin{array}{l}5847.09 \\
5847.59 \\
5848.37 \\
5848.96 \\
5849.59\end{array}$ & $\begin{array}{l}1 \\
2 \\
2 \\
1 \\
1 \text { b }\end{array}$ & $\begin{array}{l}\mathbf{B} \\
\mathbf{A} \\
\mathbf{A} \\
\mathbf{A} \\
\mathbf{E}\end{array}$ \\
\hline $\begin{array}{l}5710.22 \\
5713.85 \\
5715.41 \\
5716.69 \\
5717.11\end{array}$ & $\begin{array}{l}3 \mathbf{b} \\
1 \mathbf{b} \\
1 \\
1 \\
1\end{array}$ & $\begin{array}{l}\mathrm{B} \\
\mathrm{E} \\
\mathrm{B} \\
\mathrm{E} \\
\mathrm{E}\end{array}$ & $\begin{array}{l}5776.10 \\
5777.93 \\
5778.47 \\
5779.95 \\
5781.30\end{array}$ & $\begin{array}{l}2 \\
1 \mathrm{~b} \\
1 \\
1 \\
1\end{array}$ & $\begin{array}{l}\text { B } \\
\text { E } \\
\text { E } \\
\text { E } \\
\text { E }\end{array}$ & $\begin{array}{l}5852.01 \\
5853.00 \\
5853.50 \\
5853.75 \\
5854.42\end{array}$ & $\begin{array}{l}1 \mathrm{~b}, \mathrm{~h} \\
1 \\
1 \\
1 \\
1\end{array}$ & $\begin{array}{l}\mathbf{E} \\
\mathbf{E} \\
\mathbf{B} \\
\mathbf{D} \\
\mathbf{A}\end{array}$ \\
\hline $\begin{array}{l}5717.61 \\
5718.12 \\
5719.08 \\
5720.02 \\
5721.78\end{array}$ & $\begin{array}{l}1 \\
5 \\
3 \\
1 \\
1\end{array}$ & $\begin{array}{l}\mathbf{E} \\
\mathbf{A} \\
\mathbf{A} \\
\mathbf{E} \\
\mathbf{E}\end{array}$ & $\begin{array}{l}5783.64 \\
5784.96 \\
5788.19 \\
5788.89 \\
5791.06\end{array}$ & $\begin{array}{l}1 \\
3 \\
2 \\
1 \\
1\end{array}$ & $\begin{array}{l}\mathbf{E} \\
\text { A } \\
\text { B } \\
\text { B } \\
\text { E }\end{array}$ & $\begin{array}{l}5857.50 \\
5857.98 \\
5858.88 \\
5859.68 \\
5860.21\end{array}$ & $\begin{array}{l}2 \mathrm{Ca} ? \\
1 \\
3 \\
2 \\
1\end{array}$ & $\begin{array}{l}\mathbf{A} \\
\mathbf{E} \\
\mathbf{A} \\
\mathbf{A} \\
\mathbf{A}\end{array}$ \\
\hline $\begin{array}{l}5722.34 \\
5723.87 \\
5726.24 \\
5726.82 \\
5727.86\end{array}$ & $\begin{array}{l}1 \\
2 \\
1 \\
4 \\
2\end{array}$ & $\begin{array}{l}\mathbf{E} \\
\mathbf{B} \\
\mathbf{E} \\
\mathbf{A} \\
\mathbf{B}\end{array}$ & $\begin{array}{l}5791.47 \\
5792.90 \\
5793.97 \\
5795.15 \\
5795.64\end{array}$ & $\begin{array}{l}1 \\
1 \\
2 \\
3 \\
1 \\
1\end{array}$ & $\begin{array}{l}\mathrm{E} \\
\mathrm{E} \\
\mathrm{E} \\
\mathrm{A} \\
\mathrm{E}\end{array}$ & $\begin{array}{l}5860.60 \\
5861.15 \\
5864.03 \\
5865.03 \\
5867.05\end{array}$ & $\begin{array}{l}1 \\
2 \\
2 \\
3 \\
3\end{array}$ & $\begin{array}{l}\mathbf{E} \\
\mathbf{B} \\
\mathbf{A} \\
\mathbf{A} \\
\mathbf{A}\end{array}$ \\
\hline $\begin{array}{l}5729.30 \\
5730.29 \\
5731.09 \\
5731.77 \\
5732.90\end{array}$ & $\begin{array}{l}5 \\
2 \\
2 \\
2 \\
1 \mathrm{~b}\end{array}$ & $\begin{array}{l}\mathrm{A} \\
\mathbf{B} \\
\mathbf{E} \\
\mathbf{B} \\
\mathbf{E}\end{array}$ & $\begin{array}{l}5797.35 \\
5798.21 \\
5798.91 \\
5800.07 \\
5801.85\end{array}$ & $\begin{array}{l}1 \\
1 \mathrm{~b} \\
1 \\
3 \\
1\end{array}$ & $\begin{array}{l}\mathbf{B} \\
\mathbf{C} \\
\mathbf{E} \\
\mathbf{A} \\
\mathbf{E}\end{array}$ & $\begin{array}{l}5867.61 \\
5868.88 \\
5869.57 \\
5870.98 \\
5871.47\end{array}$ & $\begin{array}{l}2 \\
4 \\
2 \mathrm{~b}, 1 \\
3 \\
1\end{array}$ & $\begin{array}{l}\mathbf{A} \\
\mathbf{A} \\
\mathbf{A} \\
\mathbf{A} \\
\mathbf{E}\end{array}$ \\
\hline $\begin{array}{l}5734.20 \\
5734.55 \\
5736.04 \\
5737.81 \\
5738.11\end{array}$ & $\begin{array}{l}2 \\
2 \\
1 \\
1 \\
1\end{array}$ & $\begin{array}{l}\mathbf{E} \\
\text { A } \\
\text { B } \\
\text { E } \\
\mathbf{E}\end{array}$ & $\begin{array}{l}5802.50 \\
5804.01 \\
5806.41 \\
5808.78 \\
5809.24\end{array}$ & $\begin{array}{l}1 \\
6 \\
21 \\
1 \\
3\end{array}$ & $\begin{array}{l}\mathbf{E} \\
\mathbf{A} \\
\mathbf{E} \\
\mathbf{E} \\
\mathbf{A}\end{array}$ & $\begin{array}{l}5872.00 \\
5872.92 \\
5873.33 \\
5873.80 \\
5874.39\end{array}$ & $\begin{array}{l}1 \\
1 \\
2 \\
1 \\
2\end{array}$ & $\begin{array}{l}\mathrm{E} \\
\mathrm{E} \\
\mathrm{B} \\
\mathrm{C} \\
\mathrm{B}\end{array}$ \\
\hline $\begin{array}{l}5738.93 \\
5741.29 \\
5742.08 \\
5742.74 \\
5743.23\end{array}$ & $\begin{array}{l}31, \mathrm{~d} ? \\
2 \\
4 \\
2 \\
3\end{array}$ & $\begin{array}{l}\mathbf{A} \\
\mathbf{A} \\
\mathbf{A} \\
\mathbf{B} \\
\mathbf{A}\end{array}$ & $\begin{array}{l}5811.58 \\
5812.80 \\
5813.19 \\
5813.87 \\
5814.71\end{array}$ & $\begin{array}{l}4 \\
1 \\
1 \\
3 \\
1 \mathrm{~b}\end{array}$ & $\begin{array}{l}\mathbf{A} \\
\mathbf{E} \\
\mathbf{E} \\
\mathbf{A} \\
\mathbf{E}\end{array}$ & $\begin{array}{l}5874.76 \\
5875.82 \\
5876.34 \\
5876.85 \\
5877.79\end{array}$ & $\begin{array}{l}3 \\
1 \\
2 \\
1 \\
3\end{array}$ & $\begin{array}{l}\mathbf{B} \\
\mathbf{A} \\
\mathbf{A} \\
\mathbf{E} \\
\mathbf{A}\end{array}$ \\
\hline $\begin{array}{l}5744.13 \\
5744.77 \\
5745.66 \\
5746.26 \\
5746.87\end{array}$ & $\begin{array}{l}2 \\
3 \\
1 \\
1 \\
2\end{array}$ & $\begin{array}{l}\mathbf{B} \\
\mathbf{A} \\
\mathbf{B} \\
\mathbf{E} \\
\mathbf{E}\end{array}$ & $\begin{array}{l}5815.43 \\
5816.41 \\
5817.41 \\
5817.82 \\
5818.70\end{array}$ & $\begin{array}{l}3 \\
1 \\
1 \\
1 \\
2 \mathrm{~b}, 1\end{array}$ & $\begin{array}{l}\text { B } \\
\text { E } \\
\text { E } \\
\text { E } \\
\text { B }\end{array}$ & $\begin{array}{l}5878.20 \\
5878.88 \\
5879.26 \\
589.76 \\
5880.25\end{array}$ & $\begin{array}{l}1 \\
1 \\
1 \\
1 \\
2\end{array}$ & $\begin{array}{l}\mathbf{B} \\
\mathbf{B} \\
\mathbf{B} \\
\mathbf{B} \\
\mathbf{B}\end{array}$ \\
\hline $\begin{array}{l}5747.34 \\
5748.15 \\
5749.14 \\
5749.63 \\
5750.08\end{array}$ & $\begin{array}{l}1 \\
3 \\
5 \\
2 \\
1\end{array}$ & $\begin{array}{l}\mathbf{B} \\
\mathbf{A} \\
\mathbf{A} \\
\mathbf{A} \\
\mathbf{E}\end{array}$ & $\begin{array}{l}5819.26 \\
5820.36 \\
5820.56 \\
5820.95 \\
5821.73\end{array}$ & $\begin{array}{l}1 \\
3 \\
1 \\
2 \\
1\end{array}$ & $\begin{array}{l}\mathrm{E} \\
\mathrm{A} \\
\mathrm{C} \\
\mathrm{B} \\
\mathrm{E}\end{array}$ & $\begin{array}{l}5881.33 \\
5882.75 \\
5888.28 \\
5883.66 \\
5884.63\end{array}$ & $\begin{array}{l}\frac{1}{3} \\
3 \\
1 \\
1\end{array}$ & $\begin{array}{l}\mathbf{A} \\
\mathbf{A} \\
\mathbf{A} \\
\mathbf{E} \\
\mathbf{B}\end{array}$ \\
\hline $\begin{array}{l}5750.68 \\
5751.38 \\
5751.86 \\
5752.36 \\
5753.53\end{array}$ & $\begin{array}{l}1 \text { b } \\
1 \\
1 \\
1 \\
3\end{array}$ & $\begin{array}{l}\mathbf{E} \\
\mathbf{E} \\
\mathbf{E} \\
\mathbf{E} \\
\mathbf{A}\end{array}$ & $\begin{array}{l}5823.30 \\
5823.89 \\
5824.58 \\
5825.11 \\
5825.87\end{array}$ & $\begin{array}{l}3 \\
3 \\
1 \\
1 \\
4\end{array}$ & $\begin{array}{l}\mathbf{A} \\
\mathbf{A} \\
\mathbf{E} \\
\mathbf{E} \\
\mathbf{A}\end{array}$ & $\begin{array}{l}5885.08 \\
5886.22 \\
5887.89 \\
5890.50 \\
5891.52\end{array}$ & $\begin{array}{l}1 \\
3 \\
3 \\
2 \\
3\end{array}$ & $\begin{array}{l}\mathbf{E} \\
\mathbf{A} \\
\mathbf{A} \\
\mathbf{A} \\
\mathbf{A}\end{array}$ \\
\hline $\begin{array}{l}5754.10 \\
5755.21 \\
5756.12 \\
5756.62 \\
5757.50\end{array}$ & $\begin{array}{l}2 \\
2 \\
2 \\
1 \mathbf{h} \\
1\end{array}$ & $\begin{array}{l}\mathbf{B} \\
\text { B } \\
\mathbf{B} \\
\mathbf{E} \\
\mathbf{E}\end{array}$ & $\begin{array}{l}5826.75 \\
5829.13 \\
5830.72 \\
5830.95 \\
5831.81\end{array}$ & $\begin{array}{l}3 \mathrm{Er} ? \\
\frac{1}{21, n ?} \\
11 \\
1\end{array}$ & $\begin{array}{l}\mathbf{A} \\
\mathbf{E} \\
\mathbf{A} \\
\mathbf{B} \\
\mathbf{B}\end{array}$ & $\begin{array}{l}5892.69 \\
5893.60 \\
5894.86 \\
5895.54 \\
5896.67\end{array}$ & $\begin{array}{l}2 \\
1 \mathrm{~b}, \mathrm{n} ? \\
2 \\
2 \\
1 \mathrm{~b}, 1\end{array}$ & $\begin{array}{l}\mathbf{A} \\
\mathbf{A} \\
\mathbf{A} \\
\mathbf{B} \\
\mathbf{A}\end{array}$ \\
\hline $\begin{array}{l}5757.90 \\
5758.32 \\
5759.44 \\
5759.97 \\
5751.23\end{array}$ & $\begin{array}{l}1 \\
1 \\
1 \\
3 \\
1\end{array}$ & $\begin{array}{l}\mathbf{E} \\
\mathbf{B} \\
\mathbf{E} \\
\mathbf{B} \\
\mathbf{E}\end{array}$ & $\begin{array}{l}5832.21 \\
5832.65 \\
5833.14 \\
5833.71 \\
5834.55\end{array}$ & $\begin{array}{l}1 \\
1 \\
1 \\
1 \\
2\end{array}$ & $\begin{array}{l}\mathrm{E} \\
\mathrm{E} \\
\mathrm{E} \\
\mathrm{B} \\
\mathrm{B}\end{array}$ & $\begin{array}{l}5897.50 \\
5898.85 \\
5899.50 \\
5900.43 \\
5902.07\end{array}$ & $\begin{array}{l}1 \mathrm{~b}, 1 \\
2 \mathrm{~b} \\
3 \\
3 \\
2 \mathrm{~b}, 1, \mathrm{Er} ?\end{array}$ & $\begin{array}{l}\mathbf{A} \\
\mathbf{A} \\
\mathbf{A} \\
\mathbf{A} \\
\mathbf{A}\end{array}$ \\
\hline
\end{tabular}


TABLE 1.-Arc Spectrum of Neodymium-Continued

\begin{tabular}{|c|c|c|c|c|c|c|c|c|}
\hline$\lambda$ I. A. & Notes & $\begin{array}{c}\text { Probable } \\
\text { error }\end{array}$ & $\lambda$ I. A. & Notes & $\begin{array}{c}\text { Probable } \\
\text { error }\end{array}$ & $\lambda$ I. A. & Notes & $\begin{array}{c}\text { Probable } \\
\text { errox }\end{array}$ \\
\hline $\begin{array}{l}5903.12 \\
5904.82 \\
5905.24 \\
5905.80 \\
5906.28\end{array}$ & $\begin{array}{l}1 \\
1 \\
1 \\
2 \\
1\end{array}$ & $\begin{array}{l}\mathbf{A} \\
\mathbf{E} \\
\mathbf{E} \\
\mathbf{B} \\
\mathbf{B}\end{array}$ & $\begin{array}{l}5960.17 \\
5960.21 \\
5961.15 \\
5962.39 \\
5964.19\end{array}$ & $\begin{array}{l}1 \mathrm{~b} \\
3 \\
2 \\
2 \\
1 \mathrm{~b}\end{array}$ & $\begin{array}{l}\mathbf{E} \\
\mathbf{E} \\
\mathbf{B} \\
\mathbf{B} \\
\mathbf{E}\end{array}$ & $\begin{array}{l}6025.54 \\
6028.26 \\
6029.29 \\
6029.76 \\
6031.28\end{array}$ & $\begin{array}{l}2 \\
1 \\
1 \\
1 \\
4\end{array}$ & $\begin{array}{l}\mathbf{B} \\
\mathbf{E} \\
\mathbf{E} \\
\mathbf{E} \\
\mathbf{A}\end{array}$ \\
\hline $\begin{array}{l}5907.54 \\
5908.19 \\
5909.32 \\
5909.86 \\
5910.29\end{array}$ & $\begin{array}{l}1 \\
11 \\
1 \\
3 \\
21\end{array}$ & $\begin{array}{l}\mathbf{E} \\
\mathbf{B} \\
\mathbf{B} \\
\mathbf{A} \\
\mathbf{A}\end{array}$ & $\begin{array}{l}5965.38 \\
5966.01 \\
5966.36 \\
5966.74 \\
5967.25\end{array}$ & $\begin{array}{l}1 \mathrm{~b} \\
1 \\
2 \\
2 \mathrm{~b} \\
1 \mathrm{~b}\end{array}$ & $\begin{array}{l}\mathbf{E} \\
\mathbf{E} \\
\mathbf{B} \\
\mathbf{B} \\
\mathbf{E}\end{array}$ & $\begin{array}{l}6031.76 \\
6032.79 \\
6033.30 \\
6034.25 \\
6035.13\end{array}$ & $\begin{array}{l}1 \\
1 \\
4 \\
4 \\
1\end{array}$ & $\begin{array}{l}\mathbf{E} \\
\mathbf{E} \\
\mathbf{A} \\
\mathbf{A} \\
\mathbf{E}\end{array}$ \\
\hline $\begin{array}{l}5911.43 \\
5912.34 \\
5913.08 \\
5914.39 \\
5914.66\end{array}$ & $\begin{array}{l}1 \\
1 \mathrm{~b} \\
1 \\
3 \\
21, \mathrm{ES}\end{array}$ & $\begin{array}{l}\mathbf{B} \\
\mathbf{E} \\
\mathbf{E} \\
\mathbf{A} \\
\mathbf{B}\end{array}$ & $\begin{array}{l}5967.85 \\
5968.30 \\
5968.80 \\
5970.86 \\
5972.47\end{array}$ & $\begin{array}{l}\frac{1}{2} \\
1 \mathrm{~b}, \mathrm{~h} \\
1 \mathrm{~b}, \mathrm{n} ? \\
1\end{array}$ & $\begin{array}{l}\mathbf{B} \\
\mathbf{A} \\
\mathbf{E} \\
\mathbf{E} \\
\mathbf{B}\end{array}$ & $\begin{array}{l}6035.61 \\
6037.95 \\
6038.89 \\
6039.91 \\
6040.98\end{array}$ & $\begin{array}{l}1 \text { ES } \\
1 \\
1 \\
1 \\
2\end{array}$ & $\begin{array}{l}\mathbf{B} \\
\mathbf{B} \\
\mathbf{E} \\
\mathbf{C} \\
\mathbf{A}\end{array}$ \\
\hline $\begin{array}{l}5915.45 \\
5916.13 \\
5916.99 \\
5917.46 \\
5918.02\end{array}$ & $\begin{array}{l}1 \\
1 \mathrm{~b} \\
1 \\
2 \\
1\end{array}$ & $\begin{array}{l}\mathbf{E} \\
\mathbf{E} \\
\mathbf{E} \\
\mathbf{B} \\
\mathbf{E}\end{array}$ & $\begin{array}{l}5974.57 \\
5975.48 \\
5976.33 \\
5977.42 \\
5977.92\end{array}$ & $\begin{array}{l}2 \\
2 \\
2 \\
3 \mathrm{~b}, \mathrm{n?} \\
1\end{array}$ & $\begin{array}{l}\mathbf{B} \\
\mathbf{B} \\
\mathbf{B} \\
\mathbf{B} \\
\mathbf{E}\end{array}$ & $\begin{array}{l}6042.17 \\
6043.80 \\
6044.46 \\
6055.11 \\
6047.54\end{array}$ & $\begin{array}{l}1 \\
1 \\
1 \\
1 \mathrm{~b}, \mathrm{n} ? \mathrm{Cu} ? \\
1 \mathrm{~b}\end{array}$ & $\begin{array}{l}\mathbf{E} \\
\mathbf{A} \\
\mathbf{E} \\
\mathbf{B} \\
\mathbf{E}\end{array}$ \\
\hline $\begin{array}{l}5918.70 \\
5919.31 \\
5919.81 \\
5920.57 \\
5921.20\end{array}$ & $\begin{array}{l}1 \mathrm{~b} \\
1 \\
2 \\
1 \\
3\end{array}$ & $\begin{array}{l}\mathbf{E} \\
\mathbf{B} \\
\mathbf{A} \\
\mathbf{E} \\
\mathbf{A}\end{array}$ & $\begin{array}{l}5978.82 \\
5979.96 \\
5981.56 \\
5982.02 \\
5982.97\end{array}$ & $\begin{array}{l}1 \\
2 \\
1 \\
1 \\
1\end{array}$ & $\begin{array}{l}\mathbf{B} \\
\mathbf{B} \\
\mathbf{E} \\
\mathbf{B} \\
\mathbf{B}\end{array}$ & $\begin{array}{l}6048.40 \\
6049.92 \\
6050.50 \\
6051.88 \\
6053.40\end{array}$ & $\begin{array}{l}1 \mathrm{~b}, \mathrm{Cu} ? \\
1 \\
2 \\
2 \\
1\end{array}$ & $\begin{array}{l}\text { C } \\
\mathbf{D} \\
\mathbf{A} \\
\mathbf{A} \\
\mathbf{A}\end{array}$ \\
\hline $\begin{array}{l}5921.82 \\
5922.33 \\
5922.79 \\
5923.27 \\
5924.31\end{array}$ & $\begin{array}{l}1 \\
1 \\
3 \\
1 \mathrm{~b} \\
1\end{array}$ & $\begin{array}{l}\mathrm{E} \\
\mathrm{E} \\
\mathrm{A} \\
\mathrm{E} \\
\mathrm{E}\end{array}$ & $\begin{array}{l}5984.81 \\
5985.72 \\
5986.36 \\
5986.88 \\
5988.16\end{array}$ & $\begin{array}{l}1 \\
1 \\
2 \\
1 \\
2\end{array}$ & $\begin{array}{l}\mathbf{B} \\
\mathbf{E} \\
\mathbf{B} \\
\mathbf{B} \\
\mathbf{B}\end{array}$ & $\begin{array}{l}6054.47 \\
6055.26 \\
6056.99 \\
6057.30 \\
6057.59\end{array}$ & $\begin{array}{l}2 \\
1 \\
1 \\
1 \\
1\end{array}$ & $\begin{array}{l}\mathbf{B} \\
\mathbf{B} \\
\mathbf{B} \\
\mathbf{E} \\
\mathbf{E}\end{array}$ \\
\hline $\begin{array}{l}5926.00 \\
5926.50 \\
5927.94 \\
5928.93 \\
5929.79\end{array}$ & $\begin{array}{l}1 \\
1 \\
2 \\
2 \\
1 \mathrm{v}\end{array}$ & $\begin{array}{l}\mathbf{E} \\
\mathbf{E} \\
\mathbf{B} \\
\mathbf{B} \\
\mathbf{E}\end{array}$ & $\begin{array}{l}5988.81 \\
5990.21 \\
5990.70 \\
5992.40 \\
5994.04\end{array}$ & $\begin{array}{l}1 \\
2 \\
2 \\
1 \\
2\end{array}$ & $\begin{array}{l}\mathbf{E} \\
\mathbf{A} \\
\mathbf{A} \\
\mathbf{B} \\
\mathbf{A}\end{array}$ & $\begin{array}{l}6058.33 \\
6058.81 \\
6060.38 \\
6061.05 \\
6062.30\end{array}$ & $\begin{array}{l}2 \\
1 \\
1 \\
3 \\
1\end{array}$ & $\begin{array}{l}\mathbf{A} \\
\mathbf{B} \\
\mathbf{E} \\
\mathbf{B} \\
\mathbf{E}\end{array}$ \\
\hline $\begin{array}{l}5931.43 \\
5932.08 \\
5933.17 \\
5933.75 \\
5934.77\end{array}$ & $\begin{array}{l}2 \\
1 \\
1 \\
1 \\
2\end{array}$ & $\begin{array}{l}\text { B } \\
\text { B } \\
\text { B } \\
\text { E } \\
\text { B }\end{array}$ & $\begin{array}{l}5994.77 \\
5995.93 \\
5996.47 \\
5996.95 \\
5998.32\end{array}$ & $\begin{array}{l}4 \\
1 \\
4 \\
1 \\
1\end{array}$ & $\begin{array}{l}\mathbf{A} \\
\mathbf{E} \\
\mathbf{A} \\
\mathbf{A} \\
\mathbf{B} \\
\mathbf{E}\end{array}$ & $\begin{array}{l}6064.59 \\
6065.18 \\
6066.07 \\
6067.00 \\
6068.73\end{array}$ & $\begin{array}{l}1 \\
2 \\
4 \\
2 \\
3\end{array}$ & $\begin{array}{l}\mathrm{E} \\
\mathbf{B} \\
\mathrm{A} \\
\mathbf{E} \\
\mathbf{B}\end{array}$ \\
\hline $\begin{array}{l}5936.59 \\
5937.52 \\
5938.07 \\
5939.09 \\
5939.73\end{array}$ & $\begin{array}{l}1 \mathrm{~b} \\
1 \\
1 \\
1 \\
3\end{array}$ & $\begin{array}{l}\mathrm{E} \\
\mathrm{E} \\
\mathrm{E} \\
\mathrm{B} \\
\mathrm{B}\end{array}$ & $\begin{array}{l}5998.80 \\
5999.50 \\
6000.09 \\
6000.64 \\
6001.41\end{array}$ & $\begin{array}{l}1 \\
1 \\
2 \\
1 \\
1 \mathrm{~b}\end{array}$ & $\begin{array}{l}\mathbf{B} \\
\mathbf{E} \\
\mathbf{B} \\
\mathbf{E} \\
\mathbf{B}\end{array}$ & $\begin{array}{l}6069.70 \\
6070.43 \\
6071.04 \\
6071.71 \\
6073.09\end{array}$ & $\begin{array}{l}1 \\
4 \\
1\end{array}$ & $\begin{array}{l}\mathrm{E} \\
\mathrm{E} \\
\mathrm{E} \\
\mathrm{A} \\
\mathrm{E}\end{array}$ \\
\hline $\begin{array}{l}5940.00 \\
5942.04 \\
5942.68 \\
5943.21 \\
5944.51\end{array}$ & $\begin{array}{l}31 \\
1 \\
1 \\
3 \\
1\end{array}$ & $\begin{array}{l}\text { B } \\
\text { B } \\
\text { B } \\
\text { A } \\
\text { E }\end{array}$ & $\begin{array}{l}6002.45 \\
6003.95 \\
6004.33 \\
6005.43 \\
6005.90\end{array}$ & $\begin{array}{l}2 \mathrm{~b} \\
1 \\
1 \\
1 \\
1\end{array}$ & $\begin{array}{l}\mathbf{B} \\
\mathbf{B} \\
\mathbf{B} \\
\mathbf{E} \\
\mathbf{E}\end{array}$ & $\begin{array}{l}6073.96 \\
6075.20 \\
6076.23 \\
6077.23 \\
6077.42\end{array}$ & $\begin{array}{l}4 \\
1 \\
1 \\
2 \mathrm{~b}, \mathrm{n} ? \\
1\end{array}$ & $\begin{array}{l}\mathbf{A} \\
\mathbf{C} \\
\mathbf{B} \\
\mathbf{A} \\
\mathbf{E}\end{array}$ \\
\hline $\begin{array}{l}5944.90 \\
5945.63 \\
5946.29 \\
5946.70 \\
5947.36\end{array}$ & $\begin{array}{l}1 \mathrm{~b} \\
1 \\
2 \\
1 \\
2\end{array}$ & $\begin{array}{l}\mathbf{E} \\
\mathbf{E} \\
\mathbf{B} \\
\mathbf{E} \\
\mathbf{A}\end{array}$ & $\begin{array}{l}6006.44 \\
6007.67 \\
6008.82 \\
6009.29 \\
6010.86\end{array}$ & $\begin{array}{l}2 \\
4 \\
1 \\
2 \\
2\end{array}$ & $\begin{array}{l}\mathbf{B} \\
\mathrm{A} \\
\mathrm{E} \\
\mathrm{A} \\
\mathrm{B}\end{array}$ & $\begin{array}{l}6079.99 \\
6080.61 \\
6001.53 \\
6082.02 \\
6082.96\end{array}$ & $\begin{array}{l}1 \\
1 b \\
1 \\
1 \\
1 b, d ?\end{array}$ & $\begin{array}{l}\mathrm{E} \\
\mathrm{E} \\
\mathrm{E} \\
\mathrm{E} \\
\mathrm{E}\end{array}$ \\
\hline $\begin{array}{l}5948.14 \\
5948.46 \\
5949.61 \\
5950.47 \\
5950.98\end{array}$ & $\begin{array}{l}1 \\
2 \\
3 \\
2 \\
1\end{array}$ & $\begin{array}{l}\mathbf{E} \\
\mathbf{B} \\
\mathbf{B} \\
\mathbf{A} \\
\mathbf{E}\end{array}$ & $\begin{array}{l}6011.50 \\
6012.90 \\
6013.98 \\
6015.33 \\
6015.74\end{array}$ & $\begin{array}{l}2 \\
1 \\
1 \mathrm{~b}, \mathrm{~h} \\
1 \\
1\end{array}$ & $\begin{array}{l}\mathbf{A} \\
\mathbf{E} \\
\mathbf{E} \\
\mathbf{A} \\
\mathbf{E}\end{array}$ & $\begin{array}{l}6084.62 \\
6086.94 \\
6087.50 \\
6087.90 \\
6088.01\end{array}$ & $\begin{array}{l}2 \\
2 \\
1 \\
1 \\
1 \mathbf{b}\end{array}$ & $\begin{array}{l}\mathbf{B} \\
\mathbf{B} \\
\mathbf{C} \\
\mathbf{E} \\
\mathbf{E}\end{array}$ \\
\hline $\begin{array}{l}5953.05 \\
5953.53 \\
5954.47 \\
5955.16 \\
5955.88\end{array}$ & $\begin{array}{l}2 \\
1 \\
1 \\
1 \\
3\end{array}$ & $\begin{array}{l}\mathbf{B} \\
\mathbf{E} \\
\mathbf{E} \\
\mathbf{B} \\
\mathbf{A}\end{array}$ & $\begin{array}{l}6016.30 \\
6017.21 \\
6017.75 \\
6018.05 \\
6018.93\end{array}$ & $\begin{array}{l}1 \\
1 \\
1 \\
1 \\
1\end{array}$ & $\begin{array}{l}\text { B } \\
\text { B } \\
\text { B } \\
\text { E } \\
\text { E }\end{array}$ & $\begin{array}{l}\text { 6091. } 24 \\
6091.58 \\
6092.06 \\
6093.03 \\
6093.76\end{array}$ & $\begin{array}{l}2 \\
2 \\
2 \\
1 \\
1\end{array}$ & $\begin{array}{l}\mathbf{E} \\
\mathbf{B} \\
\mathbf{B} \\
\mathbf{E} \\
\mathbf{E}\end{array}$ \\
\hline $\begin{array}{l}5956.32 \\
5957.07 \\
5957.58 \\
5958.00 \\
5958.65\end{array}$ & $\begin{array}{l}1 \\
1 \\
1 \\
1 \\
1\end{array}$ & $\begin{array}{l}\mathbf{E} \\
\mathbf{E} \\
\mathbf{E} \\
\mathbf{E} \\
\mathbf{B}\end{array}$ & $\begin{array}{l}6020.23 \\
6022.04 \\
6022.75 \\
6023.45 \\
6024.65\end{array}$ & $\begin{array}{l}2 \mathrm{~b}, 1 \\
1 \\
2 \mathrm{~b}, \mathrm{~d} ? \\
2 \mathrm{~b}, \mathrm{~d} ? \\
1\end{array}$ & $\begin{array}{l}\mathbf{B} \\
\mathbf{B} \\
\mathbf{B} \\
\mathbf{B} \\
\mathbf{B}\end{array}$ & $\begin{array}{l}6094.98 \\
6096.41 \\
6097.79 \\
6099.18 \\
6100.09\end{array}$ & $\begin{array}{l}1 \\
1 \\
2 \\
1 \mathrm{y} \\
11\end{array}$ & $\begin{array}{l}\mathrm{E} \\
\mathrm{E} \\
\mathrm{C} \\
\mathrm{E} \\
\mathrm{E}\end{array}$ \\
\hline
\end{tabular}


TABLE 1.-Arc Spectrum of Neodymium-Continued

\begin{tabular}{|c|c|c|c|c|c|c|c|c|}
\hline$\lambda$ I. A. & Notes & $\begin{array}{c}\text { Probable } \\
\text { error }\end{array}$ & $\lambda$ I. A. & Notes & $\begin{array}{c}\text { Probable } \\
\text { error }\end{array}$ & $\lambda$ I. A. & Notes & $\left.\right|_{\text {error }} ^{\text {Probable }}$ \\
\hline $\begin{array}{l}6101.74 \\
6104.06 \\
6105.50 \\
6106.14 \\
6106.69\end{array}$ & $\begin{array}{l}3 \\
1 \\
1 \\
1 \\
1\end{array}$ & $\begin{array}{l}\mathbf{A} \\
\mathbf{E} \\
\mathbf{C} \\
\mathbf{E} \\
\mathbf{E}\end{array}$ & $\begin{array}{l}6221.15 \\
6222.15 \\
6223.38 \\
6226.50 \\
6227.18\end{array}$ & $\begin{array}{l}1 \mathrm{v}, \mathrm{Cu} ? \\
11 \\
31, \mathrm{~d} \\
3 \\
2\end{array}$ & $\begin{array}{l}\mathrm{E} \\
\mathrm{A} \\
\mathrm{C} \\
\mathrm{A} \\
\mathrm{A}\end{array}$ & $\begin{array}{l}6365.56 \\
6366.74 \\
6372.80 \\
6375.96 \\
6377.30\end{array}$ & $\begin{array}{l}3 \\
1 \\
2 \\
3 \\
2 n ?\end{array}$ & $\begin{array}{l}\mathrm{A} \\
\mathrm{B} \\
\mathrm{A} \\
\mathrm{A} \\
\mathrm{B}\end{array}$ \\
\hline $\begin{array}{l}6107.24 \\
6107.74 \\
6108.42 \\
6109.08 \\
6109.68\end{array}$ & $\begin{array}{l}1 \mathbf{b} \\
1 \\
4 \\
2 \\
2\end{array}$ & $\begin{array}{l}\text { B } \\
\text { E } \\
\text { B } \\
\text { B } \\
\text { C }\end{array}$ & $\begin{array}{l}6238.49 \\
6243.31 \\
6244.06 \\
6248.27 \\
6250.41\end{array}$ & $\begin{array}{l}3 \\
1 \\
3 \\
2 \\
2\end{array}$ & $\begin{array}{l}\text { A } \\
\text { B } \\
\text { B } \\
\text { A } \\
\text { B }\end{array}$ & $\begin{array}{l}6382.06 \\
6383.17 \\
6384.62 \\
6385.17 \\
6386.53\end{array}$ & $\begin{array}{l}3 \\
2 \\
1 \\
4 \\
1\end{array}$ & $\begin{array}{l}\mathbf{A} \\
\mathbf{B} \\
\mathbf{B} \\
\mathbf{A} \\
\mathbf{B}\end{array}$ \\
\hline $\begin{array}{l}6111.37 \\
6113.47 \\
6113.98 \\
6114.45 \\
6117.19\end{array}$ & $\begin{array}{l}1 \\
2 \\
1 \\
1 \\
1\end{array}$ & $\begin{array}{l}\mathbf{E} \\
\mathbf{A} \\
\mathbf{B} \\
\mathbf{B} \\
\mathbf{E}\end{array}$ & $\begin{array}{l}6251.82 \\
6257.49 \\
6258.74 \\
6260.67 \\
6263.23\end{array}$ & $\begin{array}{l}2 \\
3 \\
3 \\
1 \text { n? } \\
3\end{array}$ & $\begin{array}{l}\mathbf{A} \\
\mathbf{A} \\
\mathbf{B} \\
\mathbf{B} \\
\mathbf{A}\end{array}$ & $\begin{array}{l}6386.94 \\
6390.00 \\
6395.88 \\
6396.88 \\
6402.75\end{array}$ & $\begin{array}{l}1 \\
3 \\
1 \\
1 \\
1\end{array}$ & $\begin{array}{l}\mathbf{E} \\
\mathbf{A} \\
\mathbf{E} \\
\mathbf{A} \\
\mathbf{B}\end{array}$ \\
\hline $\begin{array}{l}6117.63 \\
6119.86 \\
6120.80 \\
6121.25 \\
6122.16\end{array}$ & $\begin{array}{l}1 \\
1 \\
1 \\
1 \\
2\end{array}$ & $\begin{array}{l}\mathbf{E} \\
\mathrm{E} \\
\mathrm{B} \\
\mathrm{E} \\
\mathrm{B}\end{array}$ & $\begin{array}{l}6269.40 \\
6270.26 \\
6271.69 \\
6277.30 \\
6278.18\end{array}$ & $\begin{array}{l}1 \\
1 \\
10 \\
3 \\
1 \mathrm{~b}\end{array}$ & $\begin{array}{l}\mathrm{A} \\
\mathrm{A} \\
\mathrm{E} \\
\mathrm{A} \\
\mathrm{A}\end{array}$ & $\begin{array}{l}6403.22 \\
6410.58 \\
6414.04 \\
6414.37 \\
6416.80\end{array}$ & $\begin{array}{l}2 \\
1 \\
1 \\
1 \\
1 \mathrm{~b}\end{array}$ & $\begin{array}{l}\text { A } \\
\text { B } \\
\text { B } \\
\text { B } \\
\mathbf{B}\end{array}$ \\
\hline $\begin{array}{l}6122.99 \\
6128.50 \\
6129.20 \\
6130.60 \\
6131.82\end{array}$ & $\begin{array}{l}2 \\
1 \\
2 \\
2 \\
2\end{array}$ & $\begin{array}{l}\mathbf{B} \\
\mathbf{E} \\
\mathbf{B} \\
\mathbf{A} \\
\mathbf{B}\end{array}$ & $\begin{array}{l}6278.93 \\
6279.36 \\
6279.66 \\
6282.00 \\
6282.47\end{array}$ & $\begin{array}{l}1 \\
1 \\
1 \\
3 \\
2\end{array}$ & $\begin{array}{l}\mathrm{B} \\
\mathrm{B} \\
\mathrm{B} \\
\mathrm{A} \\
\mathrm{A}\end{array}$ & $\begin{array}{l}6419.09 \\
6420.71 \\
6425.80 \\
6477.02 \\
6428.66\end{array}$ & $\begin{array}{l}1 \mathrm{~b} \\
1 \mathrm{~b} \\
2 \\
\frac{1}{3}\end{array}$ & $\begin{array}{l}\mathbf{E} \\
\mathbf{B} \\
\mathbf{A} \\
\mathbf{E} \\
\mathbf{A}\end{array}$ \\
\hline $\begin{array}{l}6132.62 \\
6133.51 \\
6133.92 \\
6135.21 \\
6137.37\end{array}$ & $\begin{array}{l}1 \\
3 \\
2 \\
1 \\
1\end{array}$ & $\begin{array}{l}\mathrm{E} \\
\mathrm{A} \\
\mathrm{B} \\
\mathrm{E} \\
\mathrm{E}\end{array}$ & $\begin{array}{l}6283.94 \\
6284.68 \\
6285.78 \\
6287.19 \\
6287.59\end{array}$ & $\begin{array}{l}1 \\
1 \\
2 \\
2 \\
1\end{array}$ & $\begin{array}{l}\mathbf{E} \\
\mathbf{E} \\
\mathbf{A} \\
\mathbf{A} \\
\mathbf{B}\end{array}$ & $\begin{array}{l}6429.84 \\
6431.16 \\
6431.71 \\
6432.66 \\
6433.22\end{array}$ & $\begin{array}{l}2 \\
2 \\
2 \\
3 \\
2 \mathrm{n} ?\end{array}$ & $\begin{array}{l}\mathbf{B} \\
\mathbf{B} \\
\mathbf{B} \\
\mathbf{A} \\
\mathbf{B}\end{array}$ \\
\hline $\begin{array}{l}6137.88 \\
6141.86 \\
6142.42 \\
6144.54 \\
6149.28\end{array}$ & $\begin{array}{l}1 \\
1 \\
1 \\
1 \\
4\end{array}$ & $\begin{array}{l}\mathbf{E} \\
\mathbf{B} \\
\mathbf{B} \\
\mathbf{A} \\
\mathbf{A}\end{array}$ & $\begin{array}{l}6288.03 \\
6291.99 \\
6292.84 \\
6229.59 \\
6294.42\end{array}$ & $\begin{array}{l}1 \\
2 \\
3 \\
2 \\
1\end{array}$ & $\begin{array}{l}\mathrm{A} \\
\mathrm{A} \\
\mathrm{A} \\
\mathrm{A} \\
\mathrm{E}\end{array}$ & $\begin{array}{l}6434.59 \\
6435.00 \\
6436.06 \\
6439.18 \\
6439.86\end{array}$ & $\begin{array}{l}1 \\
2 \\
1 \\
1 \\
1\end{array}$ & $\begin{array}{l}\mathbf{E} \\
\mathbf{A} \\
\mathbf{E} \\
\mathbf{B} \\
\mathbf{B}\end{array}$ \\
\hline $\begin{array}{l}6150.23 \\
6152.75 \\
6155.06 \\
6156.18 \\
6156.57\end{array}$ & $\begin{array}{l}1 \\
2 \\
4 \\
3 \\
1\end{array}$ & $\begin{array}{l}\text { B } \\
\text { B } \\
\text { B } \\
\text { A } \\
\text { B }\end{array}$ & $\begin{array}{l}6297.07 \\
6298.41 \\
6300.44 \\
6300.98 \\
6301.91\end{array}$ & $\begin{array}{l}3 \\
3 \\
1 \\
2 \\
3\end{array}$ & $\begin{array}{l}\mathbf{A} \\
\mathbf{A} \\
\mathbf{B} \\
\mathbf{A} \\
\mathbf{A}\end{array}$ & $\begin{array}{l}6442.98 \\
6445.80 \\
6448.82 \\
6451.21 \\
6452.66\end{array}$ & $\begin{array}{l}1 \\
2 \\
1 \\
2 \\
1\end{array}$ & $\begin{array}{l}\mathbf{E} \\
\mathbf{A} \\
\mathbf{E} \\
\mathbf{A} \\
\mathbf{E}\end{array}$ \\
\hline $\begin{array}{l}6157.83 \\
6159.24 \\
6159.92 \\
6161.44 \\
6162.38\end{array}$ & $\begin{array}{l}4 \\
1 \\
1 \\
4 \\
2\end{array}$ & $\begin{array}{l}\mathrm{A} \\
\mathrm{E} \\
\mathrm{E} \\
\mathrm{B} \\
\mathrm{B}\end{array}$ & $\begin{array}{l}6305.76 \\
6310.48 \\
6312.70 \\
6313.26 \\
6319.67\end{array}$ & $\begin{array}{l}1 \mathrm{n} ? \\
4 \mathrm{i} \\
1 \mathrm{~b} \\
2\end{array}$ & $\begin{array}{l}\mathbf{B} \\
\mathbf{A} \\
\mathbf{B} \\
\mathbf{A} \\
\mathbf{A}\end{array}$ & $\begin{array}{l}6455.06 \\
6457.14 \\
6457.90 \\
6458.98 \\
6463.57\end{array}$ & $\begin{array}{l}1 \\
2 \\
1 \\
1 \\
2\end{array}$ & $\begin{array}{l}\mathbf{E} \\
\mathbf{A} \\
\mathbf{B} \\
\mathbf{E} \\
\mathbf{A}\end{array}$ \\
\hline $\begin{array}{l}6163.48 \\
6164.42 \\
6165.14 \\
6165.63 \\
6166.67\end{array}$ & $\begin{array}{l}1 \\
2 \\
2 \mathrm{~b} \\
2 \\
3\end{array}$ & $\begin{array}{l}\mathrm{E} \\
\mathrm{B} \\
\mathrm{B} \\
\mathrm{B} \\
\mathrm{A}\end{array}$ & $\begin{array}{l}6321.22 \\
6322.87 \\
6326.93 \\
6328.47 \\
6331.87\end{array}$ & $\begin{array}{l}2 \\
2 \\
2 \\
2 \\
2\end{array}$ & $\begin{array}{l}\mathrm{A} \\
\mathrm{A} \\
\mathrm{A} \\
\mathrm{A} \\
\mathrm{A}\end{array}$ & $\begin{array}{l}6465.23 \\
6470.61 \\
6471.71 \\
6476.23 \\
6478.78\end{array}$ & $\begin{array}{l}2 \\
1 \mathrm{~b} \\
1 \mathrm{~b} \\
1 \\
1\end{array}$ & $\begin{array}{l}\mathbf{B} \\
\mathbf{E} \\
\mathrm{E} \\
\mathrm{E} \\
\mathbf{E}\end{array}$ \\
\hline $\begin{array}{l}6168.06 \\
6170.49 \\
6171.88 \\
6176.08 \\
6176.60\end{array}$ & $\begin{array}{l}1 \\
4 \\
1 \\
2 \\
1\end{array}$ & $\begin{array}{l}\mathrm{E} \\
\mathrm{A} \\
\mathrm{B} \\
\mathrm{B} \\
\mathrm{E}\end{array}$ & $\begin{array}{l}6333.89 \\
6338.83 \\
6340.00 \\
6341.47 \\
6343.05\end{array}$ & $\begin{array}{l}1 \\
1 \\
1 \\
4 \\
1\end{array}$ & 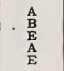 & $\begin{array}{l}6479.82 \\
6480.25 \\
6481.57 \\
6482.26 \\
6483.43\end{array}$ & $\begin{array}{l}1 \\
3 \\
1 \\
2 \\
1\end{array}$ & $\begin{array}{l}\mathrm{B} \\
\mathrm{A} \\
\mathrm{E} \\
\mathrm{E} \\
\mathrm{B}\end{array}$ \\
\hline $\begin{array}{l}6178.59 \\
6179.47 \\
6180.73 \\
6181.57 \\
6182.98\end{array}$ & $\begin{array}{l}4 \\
1 \\
1 \\
1 \\
2\end{array}$ & $\begin{array}{l}\mathbf{A} \\
\mathbf{E} \\
\mathbf{E} \\
\mathbf{E} \\
\mathbf{E}\end{array}$ & $\begin{array}{l}6347.81 \\
6348.78 \\
6349.83 \\
6351.96 \\
6353.57\end{array}$ & $\begin{array}{l}2 \\
1 \\
1 \\
2 \\
1\end{array}$ & $\begin{array}{l}\mathrm{B} \\
\mathrm{B} \\
\mathbf{E} \\
\mathrm{A} \\
\mathbf{E}\end{array}$ & $\begin{array}{l}6484.37 \\
6485.69 \\
6487.55 \\
6489.16 \\
6492.33\end{array}$ & $\begin{array}{l}1 \\
4 \\
1 \\
1 \\
2 \mathrm{Er} ?\end{array}$ & $\begin{array}{l}\mathrm{A} \\
\mathrm{A} \\
\mathrm{C} \\
\mathrm{B} \\
\mathrm{A}\end{array}$ \\
\hline $\begin{array}{l}6183.87 \\
6196.21 \\
6193.64 \\
6201.74 \\
6206.07\end{array}$ & $\begin{array}{l}3 \\
1 \\
1 \\
2 \\
1\end{array}$ & $\begin{array}{l}\text { A } \\
\text { B } \\
\text { B } \\
\text { A } \\
\text { B }\end{array}$ & $\begin{array}{l}6354.25 \\
6354.75 \\
6355.95 \\
6356.54 \\
6358.36\end{array}$ & $\begin{array}{l}1 \\
1 \\
2 \\
2 \\
1\end{array}$ & $\begin{array}{l}\mathbf{E} \\
\mathbf{A} \\
\mathbf{A} \\
\mathbf{A} \\
\mathbf{E}\end{array}$ & $\begin{array}{l}6495.59 \\
6500.15 \\
6501.11 \\
6501.86 \\
6504.45\end{array}$ & $\begin{array}{l}1 \\
2 \\
1 \mathrm{~b} \\
\frac{1}{2}\end{array}$ & $\begin{array}{l}\mathrm{E} \\
\mathrm{A} \\
\mathbf{E} \\
\mathrm{E} \\
\mathrm{A}\end{array}$ \\
\hline $\begin{array}{l}6207.96 \\
6208.23 \\
6212.79 \\
6216.71 \\
6218.16\end{array}$ & $\begin{array}{l}1 \\
3 \mathrm{v} \\
1 \\
2 \\
1\end{array}$ & $\begin{array}{l}\mathbf{E} \\
\mathbf{A} \\
\mathbf{E} \\
\mathbf{A} \\
\mathbf{A}\end{array}$ & $\begin{array}{l}6360.86 \\
6361.44 \\
6362.10 \\
6363.94 \\
6364.94\end{array}$ & $\begin{array}{l}1 \\
3 \\
3 \\
1 \\
1\end{array}$ & $\begin{array}{l}\mathrm{E} \\
\mathrm{A} \\
\mathrm{A} \\
\mathrm{B} \\
\mathrm{E}\end{array}$ & $\begin{array}{l}6504.92 \\
6507.30 \\
6514.93 \\
6519.86 \\
6523.12\end{array}$ & $\begin{array}{l}1 \\
1 \\
2 \\
1 \\
2\end{array}$ & $\begin{array}{l}\mathrm{A} \\
\mathrm{A} \\
\mathrm{A} \\
\mathrm{A} \\
\mathrm{A} \\
\mathrm{A}\end{array}$ \\
\hline
\end{tabular}


TABLE 1.-Arc Spectrum of Neodymium-Continued

\begin{tabular}{|c|c|c|c|c|c|c|c|c|}
\hline$\lambda$ I. A. & Notes & $\begin{array}{c}\text { Probable } \\
\text { error }\end{array}$ & $\lambda$ I. A. & Notes & $\begin{array}{c}\text { Probable } \\
\text { error }\end{array}$ & $\lambda$ I. A. & Notes & $\begin{array}{c}\text { Probable } \\
\text { error }\end{array}$ \\
\hline $\begin{array}{l}6539.91 \\
6541.09 \\
6553.74 \\
6547.72 \\
6549.50\end{array}$ & $\begin{array}{l}2 \\
1 \\
1 \\
1 \\
2 \\
2\end{array}$ & $\begin{array}{l}\mathrm{A} \\
\mathrm{E} \\
\mathrm{E} \\
\mathrm{A} \\
\mathrm{A}\end{array}$ & $\begin{array}{l}6709.06 \\
6712.27 \\
6713.26 \\
6714.15 \\
6718.32\end{array}$ & $\begin{array}{l}1 \\
2 \\
1 \\
1 \\
1\end{array}$ & $\begin{array}{l}\mathrm{E} \\
\mathrm{A} \\
\mathrm{B} \\
\mathrm{B} \\
\mathrm{E}\end{array}$ & $\begin{array}{l}7024.58 \\
7033.21 \\
7037.30 \\
7066.89 \\
7089.84\end{array}$ & $\begin{array}{l}2 \\
1 \\
3 \\
3 \\
1\end{array}$ & $\begin{array}{l}\mathbf{B} \\
\mathbf{E} \\
\mathbf{A} \\
\mathbf{A} \\
\mathbf{A} \\
\mathbf{E}\end{array}$ \\
\hline $\begin{array}{l}6550.18 \\
6553.06 \\
6558.95 \\
6560.27 \\
6563.94\end{array}$ & $\begin{array}{l}2 \\
2 \\
2 \\
1 \\
1 \mathrm{~b}\end{array}$ & $\begin{array}{l}\mathbf{A} \\
\mathbf{A} \\
\mathbf{A} \\
\mathbf{E} \\
\mathbf{E}\end{array}$ & $\begin{array}{l}6719.92 \\
6722.72 \\
6723.46 \\
6725.26 \\
6727.70\end{array}$ & $\begin{array}{l}1 \\
1 \text { b } \\
1 \\
1 \\
3\end{array}$ & $\begin{array}{l}\mathbf{E} \\
\mathbf{E} \\
\mathbf{B} \\
\mathbf{B} \\
\mathbf{A}\end{array}$ & $\begin{array}{l}7092.64 \\
7993.93 \\
7100.50 \\
7113.80 \\
7116.84\end{array}$ & $\begin{array}{l}1 \\
1 \\
1 \\
1 \\
1 b \\
1 b\end{array}$ & $\begin{array}{l}\mathbf{E} \\
\mathbf{C} \\
\mathbf{E} \\
\mathbf{E} \\
\mathbf{E}\end{array}$ \\
\hline $\begin{array}{l}6568.45 \\
6569.56 \\
6571.80 \\
6572.66 \\
6575.96\end{array}$ & $\begin{array}{l}1 \\
1 \\
1 \\
2 \mathrm{~b} \\
1\end{array}$ & $\begin{array}{l}\text { B } \\
\text { B } \\
\text { B } \\
\text { B } \\
\text { E }\end{array}$ & $\begin{array}{l}6728.93 \\
6734.57 \\
6775.04 \\
6737.79 \\
6739.16\end{array}$ & $\begin{array}{l}2 \\
1 \\
1 \\
3 \\
1\end{array}$ & $\begin{array}{l}\mathrm{A} \\
\mathbf{E} \\
\mathbf{B} \\
\mathrm{A} \\
\mathbf{B}\end{array}$ & $\begin{array}{l}7129.35 \\
7142.04 \\
7143.72 \\
7148.23 \\
7150.93\end{array}$ & $\begin{array}{l}3 \\
1 \\
1 \\
1 \\
1\end{array}$ & $\begin{array}{l}\mathbf{A} \\
\mathbf{E} \\
\mathbf{E} \\
\mathbf{E} \\
\mathbf{E}\end{array}$ \\
\hline $\begin{array}{l}6577.73 \\
6580.81 \\
6580.99 \\
6581.84 \\
6582.61\end{array}$ & $\begin{array}{l}1 \\
1 \nabla \\
2 \\
1 \\
1\end{array}$ & $\begin{array}{l}\text { C } \\
\text { B } \\
\text { E } \\
\text { B } \\
\mathbf{E}\end{array}$ & $\begin{array}{l}6740.05 \\
6742.50 \\
6745.18 \\
6748.18 \\
6753.60\end{array}$ & $\begin{array}{l}4 \\
2 \\
1 \\
1 \mathrm{~b} \\
1\end{array}$ & $\begin{array}{l}\text { A } \\
\text { B } \\
\text { B } \\
\text { C } \\
\text { E }\end{array}$ & $\begin{array}{l}7157.84 \\
7170.36 \\
7183.60 \\
7185.01 \\
7189.42\end{array}$ & $\begin{array}{l}11, \mathrm{n} ? \\
1 \mathrm{~b} \\
1 \\
\frac{1}{3}\end{array}$ & $\begin{array}{l}\mathbf{E} \\
\mathbf{E} \\
\mathbf{E} \\
\mathbf{E} \\
\mathbf{B}\end{array}$ \\
\hline $\begin{array}{l}6583.47 \\
6584.59 \\
6585.70 \\
6588.02 \\
6589.04\end{array}$ & $\begin{array}{l}1 \text { Er? Cu? } \\
1 \\
2 \\
2 \\
1\end{array}$ & $\begin{array}{l}\mathrm{A} \\
\mathrm{E} \\
\mathrm{A} \\
\mathrm{A} \\
\mathrm{C}\end{array}$ & $\begin{array}{l}6763.01 \\
6763.78 \\
6764.51 \\
6765.78 \\
6770.94\end{array}$ & $\begin{array}{l}2 \\
2 \\
2 \\
1 \\
1\end{array}$ & $\begin{array}{l}\mathbf{A} \\
\mathbf{A} \\
\mathbf{A} \\
\mathbf{E} \\
\mathbf{E}\end{array}$ & $\begin{array}{l}7192.01 \\
7198.83 \\
7215.32 \\
7235.24 \\
7236.52\end{array}$ & $\begin{array}{l}2 \\
2 \\
1 \\
1 \\
3\end{array}$ & $\begin{array}{l}\mathrm{A} \\
\mathrm{E} \\
\mathrm{E} \\
\mathrm{E} \\
\mathrm{B}\end{array}$ \\
\hline $\begin{array}{l}6589.64 \\
6590.94 \\
5591.40 \\
6592.66 \\
6595.00\end{array}$ & $\begin{array}{l}1 \\
1 \\
1 \\
1 \\
1\end{array}$ & $\begin{array}{l}\mathbf{A} \\
\mathbf{E} \\
\mathrm{A} \\
\mathbf{E} \\
\mathrm{A}\end{array}$ & $\begin{array}{l}6780.61 \\
6782.50 \\
6789.10 \\
6790.40 \\
6791.67\end{array}$ & $\begin{array}{l}2 \\
1 \\
1 \\
4 \\
1\end{array}$ & $\begin{array}{l}\mathbf{B} \\
\mathbf{A} \\
\mathbf{B} \\
\mathbf{A} \\
\mathbf{C}\end{array}$ & $\begin{array}{l}7242.16 \\
7243.23 \\
7261.71 \\
7266.22 \\
7268.31\end{array}$ & $\begin{array}{l}1 \\
1 \\
1 \\
1 \\
1\end{array}$ & $\begin{array}{l}\mathbf{E} \\
\mathbf{E} \\
\mathbf{E} \\
\mathbf{E} \\
\mathbf{E}\end{array}$ \\
\hline $\begin{array}{l}6596.35 \\
6601.75 \\
6503.68 \\
6604.44 \\
6609.30\end{array}$ & $\begin{array}{l}1 \\
2 \\
1 \\
1 \mathrm{~b} \\
1\end{array}$ & $\begin{array}{l}\mathbf{B} \\
\mathbf{A} \\
\mathbf{E} \\
\mathbf{C} \\
\mathbf{E}\end{array}$ & $\begin{array}{l}6792.28 \\
6795.34 \\
6795.48 \\
6801.34 \\
6803.97\end{array}$ & $\begin{array}{l}1 \\
1 \\
1 \\
2 \mathrm{~h} \\
4\end{array}$ & $\begin{array}{l}\mathbf{B} \\
\mathbf{B} \\
\mathbf{E} \\
\mathbf{B} \\
\mathbf{A}\end{array}$ & $\begin{array}{l}7272.26 \\
7279.36 \\
7285.30 \\
7228.52 \\
7291.38\end{array}$ & $\begin{array}{l}1 \\
1 \\
3 \\
3 \\
2 \\
2\end{array}$ & $\begin{array}{l}\mathbf{E} \\
\mathbf{E} \\
\mathbf{A} \\
\mathbf{A} \\
\mathbf{A}\end{array}$ \\
\hline $\begin{array}{l}6609.68 \\
6611.99 \\
6615.88 \\
6616.89 \\
6618.49\end{array}$ & $\begin{array}{l}1 \\
3 \\
1 \\
1 \\
3\end{array}$ & $\begin{array}{l}\mathbf{E} \\
\mathbf{A} \\
\mathbf{B} \\
\mathbf{E} \\
\mathbf{A}\end{array}$ & $\begin{array}{l}6806.24 \\
6812.30 \\
6816.00 \\
6822.91 \\
6825.35\end{array}$ & $\begin{array}{l}1 \\
1 \mathrm{~b} \\
2 \mathrm{~b} \\
1 \\
1\end{array}$ & $\begin{array}{l}\text { B } \\
\text { B } \\
\text { A } \\
\text { B } \\
\text { A }\end{array}$ & $\begin{array}{l}7298.77 \\
7308.78 \\
7311.91 \\
7313.70 \\
7316.80\end{array}$ & $\begin{array}{l}1 \\
1 \\
1 \\
1 \\
3\end{array}$ & $\begin{array}{l}\mathbf{B} \\
\mathbf{A} \\
\mathbf{E} \\
\mathbf{E} \\
\mathbf{A}\end{array}$ \\
\hline $\begin{array}{l}6619.34 \\
6624.26 \\
6625.40 \\
6630.14 \\
6636.15\end{array}$ & $\begin{array}{l}3 \\
1 \text { b } \\
1 \\
4 \\
1\end{array}$ & $\begin{array}{l}\mathrm{A} \\
\mathrm{E} \\
\mathbf{E} \\
\mathrm{A} \\
\mathrm{C}\end{array}$ & $\begin{array}{l}6842.67 \\
6846.73 \\
6884.30 \\
6852.23 \\
6853.72\end{array}$ & $\begin{array}{l}2 \\
4 \\
1 \mathrm{~b} \\
2 \\
1\end{array}$ & $\begin{array}{l}\mathrm{A} \\
\mathrm{A} \\
\mathrm{E} \\
\mathrm{B} \\
\mathrm{E}\end{array}$ & $\begin{array}{l}7321.43 \\
7323.10 \\
7324.63 \\
7337.89 \\
7330.80\end{array}$ & $\begin{array}{l}2 \\
2 \\
1 \\
1 \\
1\end{array}$ & $\begin{array}{l}\text { A } \\
\text { A } \\
\text { E } \\
\text { A } \\
\text { E }\end{array}$ \\
\hline $\begin{array}{l}6637.96 \\
6650.57 \\
6655.64 \\
6659.47 \\
6650.16\end{array}$ & $\begin{array}{l}3 \\
4 \\
4 \\
1 \\
2\end{array}$ & $\begin{array}{l}\mathbf{A} \\
\mathbf{A} \\
\mathbf{A} \\
\mathbf{A} \\
\mathbf{A}\end{array}$ & $\begin{array}{l}6855.69 \\
6856.96 \\
6865.52 \\
6868.61 \\
6873.78\end{array}$ & $\begin{array}{l}1 \\
1 \\
1 \\
1 \\
1\end{array}$ & $\begin{array}{l}\mathbf{E} \\
\mathrm{B} \\
\mathbf{E} \\
\mathrm{E} \\
\mathrm{E}\end{array}$ & $\begin{array}{l}7333.19 \\
7334.56 \\
7334.49 \\
7336.43 \\
7350.32\end{array}$ & $\begin{array}{l}1 \\
2 \\
1 \\
1 \\
1\end{array}$ & $\begin{array}{l}\mathrm{B} \\
\mathrm{A} \\
\mathrm{A} \\
\mathrm{A} \\
\mathrm{B} \\
\mathbf{C}\end{array}$ \\
\hline $\begin{array}{l}6662.55 \\
6664.61 \\
6665.78 \\
6657.54 \\
6669.61\end{array}$ & $\begin{array}{l}1 \\
1 \\
1 \\
11 \\
2\end{array}$ & $\begin{array}{l}\mathbf{E} \\
\mathbf{B} \\
\mathbf{E} \\
\mathbf{B} \\
\mathbf{A}\end{array}$ & $\begin{array}{l}6874.66 \\
6876.00 \\
6887.41 \\
6886.86 \\
6896.65\end{array}$ & $\begin{array}{l}2 \\
2 \\
1 \\
2 \\
2\end{array}$ & $\begin{array}{l}\mathbf{A} \\
\mathbf{A} \\
\mathbf{B} \\
\mathbf{B} \\
\mathbf{A}\end{array}$ & $\begin{array}{l}7354.09 \\
7357.16 \\
7360.53 \\
7363.28 \\
7366.24\end{array}$ & $\begin{array}{l}\frac{1}{2} \\
2 \\
1 \\
1\end{array}$ & $\begin{array}{l}\text { B } \\
\text { A } \\
\text { B } \\
\text { B } \\
\text { E }\end{array}$ \\
\hline $\begin{array}{l}6670.37 \\
6671.10 \\
6673.89 \\
6675.07 \\
6675.96\end{array}$ & $\begin{array}{l}2 \\
1 \\
1 \\
1 \mathrm{n} ? \\
1\end{array}$ & $\begin{array}{l}\mathbf{A} \\
\mathbf{E} \\
\mathbf{B} \\
\mathbf{B} \\
\mathbf{E}\end{array}$ & $\begin{array}{l}6897.29 \\
6900.40 \\
6901.33 \\
6923.86 \\
6926.87\end{array}$ & $\begin{array}{l}1 \\
4 \\
2 \\
2 \\
2 \\
2\end{array}$ & $\begin{array}{l}\mathbf{A} \\
\text { A } \\
\text { B } \\
\text { A } \\
\mathbf{A}\end{array}$ & $\begin{array}{l}7374.04 \\
7376.48 \\
7378.26 \\
7381.81 \\
7383.52\end{array}$ & $\begin{array}{l}2 \\
1 \\
2 \\
3 \\
1 \\
1\end{array}$ & $\begin{array}{l}\text { A } \\
\text { B } \\
\text { B } \\
\text { A } \\
\text { B }\end{array}$ \\
\hline $\begin{array}{l}6678.52 \\
6679.75 \\
6680.14 \\
6681.12 \\
6683.52\end{array}$ & $\begin{array}{l}2 \\
1 \\
2 \\
1 \\
1\end{array}$ & $\begin{array}{l}\mathbf{A} \\
\mathbf{A} \\
\mathbf{A} \\
\mathbf{E} \\
\mathbf{E}\end{array}$ & $\begin{array}{l}6927.99 \\
6932.16 \\
6993.44 \\
6938.67 \\
6940.14\end{array}$ & $\begin{array}{l}1 \\
1 \\
1 \mathrm{~b} \\
1 \\
2\end{array}$ & $\begin{array}{l}\mathbf{E} \\
\mathbf{A} \\
\mathbf{E} \\
\mathbf{A} \\
\mathbf{A}\end{array}$ & $\begin{array}{l}7390.18 \\
7391.25 \\
7394.40 \\
7397.13 \\
7399.46\end{array}$ & $\begin{array}{l}1 \\
1 \\
1 \\
1 \\
1\end{array}$ & $\begin{array}{l}\text { B } \\
\text { B } \\
\text { B } \\
\text { E } \\
\mathbf{E}\end{array}$ \\
\hline $\begin{array}{l}6590.15 \\
6693.77 \\
6695.62 \\
6698.65 \\
6702.70\end{array}$ & $\begin{array}{l}1 \\
1 \\
1 \\
2 \\
1\end{array}$ & $\begin{array}{l}\mathrm{E} \\
\mathrm{E} \\
\mathrm{E} \\
\mathrm{A} \\
\mathrm{B}\end{array}$ & $\begin{array}{l}6941.39 \\
6964.64 \\
6982.65 \\
7010.80 \\
7020.92\end{array}$ & $\begin{array}{l}2 \\
2 \mathrm{~b} \\
1 \\
\frac{1}{2} \mathrm{~b}\end{array}$ & $\begin{array}{l}\mathbf{A} \\
\mathbf{A} \\
\mathbf{E} \\
\mathbf{E} \\
\mathbf{A}\end{array}$ & $\begin{array}{l}7401.31 \\
7403.38 \\
7404.78 \\
7406.62 \\
7409.73\end{array}$ & $\begin{array}{l}3 \\
1 \\
2 \\
3 \\
2\end{array}$ & $\begin{array}{l}\mathbf{A} \\
\mathbf{C} \\
\mathbf{A} \\
\mathbf{A} \\
\mathbf{A}\end{array}$ \\
\hline
\end{tabular}


TABLE 1.-Arc Spectrum of Neodymium-Continued

\begin{tabular}{|c|c|c|c|c|c|c|c|c|}
\hline 入 1. A. & Notes & $\begin{array}{c}\text { Probable } \\
\text { error }\end{array}$ & $\lambda$ I. A. & Notes & $\begin{array}{c}\text { Probable } \\
\text { error }\end{array}$ & $\lambda$ I. A. & Notes & $\begin{array}{c}\text { Probable } \\
\text { error }\end{array}$ \\
\hline $\begin{array}{l}7411.20 \\
7418.18 \\
7422.24 \\
7424.48 \\
7427.41\end{array}$ & $\begin{array}{l}2 \\
4 \\
1 \\
1 \\
3\end{array}$ & $\begin{array}{l}\mathbf{A} \\
\mathbf{A} \\
\mathbf{A} \\
\mathbf{B} \\
\mathbf{A}\end{array}$ & $\begin{array}{l}7621.69 \\
7623.93 \\
7624.82 \\
7626.40 \\
7628.95\end{array}$ & $\begin{array}{l}1 \\
1 \\
1 \\
1 \\
1\end{array}$ & $\begin{array}{l}\mathbf{B} \\
\mathbf{E} \\
\mathbf{E} \\
\mathbf{A} \\
\mathbf{A}\end{array}$ & $\begin{array}{l}7845.51 \\
7851.99 \\
7853.24 \\
7857.51 \\
7858.94\end{array}$ & $\begin{array}{l}2 \\
1 \\
2 \\
1 \\
1\end{array}$ & $\begin{array}{l}\text { A } \\
\text { B } \\
\text { A } \\
\text { B } \\
\text { E }\end{array}$ \\
\hline $\begin{array}{l}7430.00 \\
7432.20 \\
7433.31 \\
7436.05 \\
7437.56\end{array}$ & $\begin{array}{l}1 \\
1 \\
1 \\
1 \\
1\end{array}$ & $\begin{array}{l}\stackrel{\mathrm{B}}{\mathrm{D}} \\
\mathrm{D} \\
\mathrm{C} \\
\mathrm{B} \\
\mathrm{B}\end{array}$ & $\begin{array}{l}7632.82 \\
7636.77 \\
7637.80 \\
7639.79 \\
7645.98\end{array}$ & $\begin{array}{l}2 \\
1 \\
1 \\
3 \\
3\end{array}$ & $\begin{array}{l}\text { A } \\
\text { C } \\
\text { E } \\
\text { A } \\
\text { A }\end{array}$ & $\begin{array}{l}7859.90 \\
7862.84 \\
7867.10 \\
7869.51 \\
7872.03\end{array}$ & $\begin{array}{l}1 \\
4 \\
1 \\
1 \\
2\end{array}$ & $\begin{array}{l}\mathbf{E} \\
\mathbf{A} \\
\mathbf{E} \\
\mathbf{E} \\
\mathbf{A}\end{array}$ \\
\hline $\begin{array}{l}7441.57 \\
7442.82 \\
7445.57 \\
7448.76 \\
7451.11\end{array}$ & $\begin{array}{l}1 \\
1 \\
1 \\
4 \\
1 \mathrm{~b}\end{array}$ & $\begin{array}{l}\mathrm{E} \\
\mathrm{E} \\
\mathrm{A} \\
\mathrm{A} \\
\mathrm{E}\end{array}$ & $\begin{array}{l}7654.53 \\
7656.11 \\
7657.35 \\
7659.35 \\
7651.17\end{array}$ & $\begin{array}{l}1 \\
1 \\
1 \\
1 \\
1\end{array}$ & $\begin{array}{l}\mathbf{B} \\
\mathbf{B} \\
\mathbf{E} \\
\mathbf{E} \\
\mathbf{A}\end{array}$ & $\begin{array}{l}7874.08 \\
7877.67 \\
7880.23 \\
781.45 \\
7886.60\end{array}$ & $\begin{array}{l}1 \\
1 \\
1 \\
1 \\
2\end{array}$ & $\begin{array}{l}\text { B } \\
\text { E } \\
\mathbf{E} \\
\text { B } \\
\mathbf{A}\end{array}$ \\
\hline $\begin{array}{l}7456.91 \\
7458.98 \\
7466.46 \\
7468.16 \\
7472.48\end{array}$ & $\begin{array}{l}1 \\
1 \\
1 \\
2 \\
1\end{array}$ & $\begin{array}{l}\mathrm{E} \\
\mathrm{C} \\
\mathrm{E} \\
\mathrm{B} \\
\mathrm{B}\end{array}$ & $\begin{array}{l}7663.48 \\
7673.88 \\
7675.10 \\
7677.25 \\
7678.78\end{array}$ & $\begin{array}{l}3 \\
1 \\
1 \\
1 \\
1\end{array}$ & $\begin{array}{l}\mathbf{A} \\
\mathbf{E} \\
\mathbf{E} \\
\mathbf{E} \\
\mathbf{E}\end{array}$ & $\begin{array}{l}7896.50 \\
7898.29 \\
7900.38 \\
7906.03 \\
7908.51\end{array}$ & $\begin{array}{l}2 \\
1 \\
2 \\
2 \\
1\end{array}$ & $\begin{array}{l}\mathbf{A} \\
\mathbf{E} \\
\mathbf{A} \\
\mathbf{A} \\
\mathbf{E}\end{array}$ \\
\hline $\begin{array}{l}7475.55 \\
7476.67 \\
7478.62 \\
7481.37 \\
7483.88\end{array}$ & $\begin{array}{l}2 \\
2 \\
1 \\
2 \\
2\end{array}$ & $\begin{array}{l}\mathrm{A} \\
\mathrm{A} \\
\mathrm{B} \\
\mathrm{A} \\
\mathrm{A}\end{array}$ & $\begin{array}{l}7682.59 \\
7684.83 \\
7690.02 \\
7691.76 \\
7693.97\end{array}$ & $\begin{array}{l}1 \\
2 \\
1 \\
1 \\
1\end{array}$ & $\begin{array}{l}\mathbf{C} \\
\mathbf{A} \\
\mathbf{A} \\
\mathbf{E} \\
\mathbf{E}\end{array}$ & $\begin{array}{l}7915.22 \\
7917.01 \\
7921.63 \\
7923.12 \\
7925.03\end{array}$ & $\begin{array}{l}1 \\
3 \\
1 \\
2 \\
3\end{array}$ & $\begin{array}{l}\mathbf{E} \\
\mathbf{A} \\
\mathbf{B} \\
\mathbf{A} \\
\mathbf{A}\end{array}$ \\
\hline $\begin{array}{l}7485.03 \\
7487.48 \\
7489.49 \\
7491.56 \\
7493.39\end{array}$ & $\begin{array}{l}1 \\
1 \text { b } \\
1 \\
1 \\
1\end{array}$ & $\begin{array}{l}\mathrm{E} \\
\mathrm{E} \\
\mathrm{B} \\
\mathrm{E} \\
\mathrm{E}\end{array}$ & $\begin{array}{l}7695.46 \\
7696.61 \\
7698.80 \\
7705.70 \\
7711.58\end{array}$ & $\begin{array}{l}1 \\
4 \\
2 \\
2 \\
2\end{array}$ & $\begin{array}{l}\mathbf{B} \\
\mathbf{A} \\
\mathbf{A} \\
\mathbf{A} \\
\mathbf{A}\end{array}$ & $\begin{array}{l}7927.43 \\
7943.05 \\
7947.95 \\
7949.65 \\
7951.54\end{array}$ & $\begin{array}{l}2 \\
1 \\
1 \\
3 \\
1\end{array}$ & $\begin{array}{l}\text { A } \\
\text { E } \\
\text { A } \\
\text { A } \\
\text { E }\end{array}$ \\
\hline $\begin{array}{l}7494.93 \\
7507.81 \\
7511.16 \\
7513.84 \\
7514.44\end{array}$ & $\begin{array}{l}1 \\
1 \\
4 \\
5 \\
1\end{array}$ & $\begin{array}{l}\mathrm{E} \\
\mathrm{C} \\
\mathrm{A} \\
\mathrm{B} \\
\mathrm{E}\end{array}$ & $\begin{array}{l}7715.17 \\
7718.15 \\
7721.25 \\
7724.84 \\
7727.76\end{array}$ & $\begin{array}{l}1 \\
3 \\
1 \\
2 \\
1\end{array}$ & $\begin{array}{l}\mathrm{E} \\
\mathrm{A} \\
\mathrm{B} \\
\mathrm{A} \\
\mathrm{A} \\
\mathrm{E}\end{array}$ & $\begin{array}{l}7955.38 \\
7957.47 \\
7958.93 \\
7963.95 \\
7965.69\end{array}$ & $\begin{array}{l}2 \\
2 \\
4 \\
1 \\
4\end{array}$ & $\begin{array}{l}\mathbf{B} \\
\mathbf{A} \\
\mathbf{A} \\
\mathbf{B} \\
\mathbf{A}\end{array}$ \\
\hline $\begin{array}{l}7516.01 \\
7523.09 \\
7526.49 \\
7529.01 \\
7534.12\end{array}$ & $\begin{array}{l}2 \\
1 \\
3 \\
4 \\
1\end{array}$ & $\begin{array}{l}\mathrm{A} \\
\mathrm{B} \\
\mathrm{A} \\
\mathrm{A} \\
\mathrm{E}\end{array}$ & $\begin{array}{l}7729.21 \\
7736.01 \\
7740.80 \\
7743.87 \\
7749.02\end{array}$ & $\begin{array}{l}1 \\
1 \\
2 \\
2 \\
2\end{array}$ & $\begin{array}{l}\mathbf{E} \\
\mathbf{C} \\
\mathbf{A} \\
\mathbf{A} \\
\mathbf{A}\end{array}$ & $\begin{array}{l}7967.88 \\
7970.64 \\
7972.05 \\
7974.47 \\
7982.34\end{array}$ & $\begin{array}{l}1 \\
1 \\
1 \\
1 \\
4 \mathrm{~b}\end{array}$ & $\begin{array}{l}\mathrm{C} \\
\mathrm{E} \\
\mathbf{E} \\
\mathbf{E} \\
\mathrm{A}\end{array}$ \\
\hline $\begin{array}{l}7535.79 \\
7538.27 \\
7540.98 \\
7543.31 \\
7544.70\end{array}$ & $\begin{array}{l}2 \\
4 \\
2 \\
1 \\
1\end{array}$ & $\begin{array}{l}\mathrm{A} \\
\mathrm{A} \\
\mathrm{A} \\
\mathrm{E} \\
\mathrm{B}\end{array}$ & $\begin{array}{l}7750.97 \\
7753.56 \\
7755.38 \\
7757.45 \\
7758.94\end{array}$ & $\begin{array}{l}3 \\
1 \\
1 \\
1 \\
1\end{array}$ & $\begin{array}{l}\mathbf{A} \\
\mathbf{A} \\
\mathbf{B} \\
\mathbf{B} \\
\mathbf{A}\end{array}$ & $\begin{array}{l}7984.75 \\
7987.04 \\
7988.72 \\
7989.79 \\
7991.22\end{array}$ & $\begin{array}{l}1 \\
1 \\
1 \\
1 \\
1\end{array}$ & $\begin{array}{l}\mathrm{B} \\
\mathrm{B} \\
\mathrm{E} \\
\mathrm{E} \\
\mathrm{E} \\
\mathrm{E}\end{array}$ \\
\hline $\begin{array}{l}7545.89 \\
7547.00 \\
7549.20 \\
7550.90 \\
7553.21\end{array}$ & $\begin{array}{l}\begin{array}{l}1 \\
3 \\
1 \\
1 \\
1\end{array} \\
d ?\end{array}$ & $\begin{array}{l}\mathrm{E} \\
\mathrm{A} \\
\mathrm{B} \\
\mathrm{B} \\
\mathrm{B}\end{array}$ & $\begin{array}{l}7765.39 \\
7767.00 \\
7768.02 \\
7733.14 \\
7775.48\end{array}$ & $\begin{array}{l}1 \\
1 \\
2 \\
3 \\
1\end{array}$ & $\begin{array}{l}\mathrm{C} \\
\mathrm{A} \\
\mathrm{A} \\
\mathrm{A} \\
\mathrm{E}\end{array}$ & $\begin{array}{l}7996.12 \\
8000.75 \\
8005.34 \\
8007.72 \\
8010.22\end{array}$ & $\begin{array}{ll}1 & \\
4 & \\
1 & 1 \\
3 & \\
1 & b\end{array}$ & $\begin{array}{l}\mathbf{B} \\
\text { A } \\
\mathbf{B} \\
\mathbf{A} \\
\mathbf{C}\end{array}$ \\
\hline $\begin{array}{l}7555.63 \\
7563.21 \\
7566.00 \\
7575.82 \\
7577.54\end{array}$ & $\begin{array}{l}1 \\
2 \\
2 \\
1 \\
2 \\
2\end{array}$ & $\begin{array}{l}\mathbf{C} \\
\mathbf{A} \\
\mathbf{A} \\
\mathbf{E} \\
\mathbf{A}\end{array}$ & $\begin{array}{l}7777.11 \\
7779.87 \\
7785.88 \\
7787.37 \\
7792.27\end{array}$ & $\begin{array}{l}1 \\
1 \\
1 \\
2 \\
3\end{array}$ & $\begin{array}{l}\mathbf{C} \\
\mathbf{C} \\
\mathbf{B} \\
\mathbf{A} \\
\mathbf{A}\end{array}$ & $\begin{array}{l}8015.32 \\
8020.08 \\
8031.44 \\
8033.29 \\
8034.60\end{array}$ & $\begin{array}{l}1 \\
2 \\
1 \\
1 \\
1\end{array}$ & $\begin{array}{l}\mathrm{C} \\
\mathrm{B} \\
\mathbf{E} \\
\mathbf{E} \\
\mathbf{E}\end{array}$ \\
\hline $\begin{array}{l}7579.18 \\
7580.88 \\
7583.84 \\
7587.65 \\
7590.49\end{array}$ & $\begin{array}{l}2 \\
1 \\
1 \\
3 \\
3 \mathrm{~b}, \mathrm{v}\end{array}$ & $\begin{array}{l}\mathbf{A} \\
\mathbf{E} \\
\mathbf{E} \\
\mathbf{A} \\
\mathbf{B}\end{array}$ & $\begin{array}{l}\text { 7796. } 37 \\
\text { 7797. } 32 \\
\text { 7804. } 39 \\
\text { 7808. } 50 \\
\text { 7811. } 26\end{array}$ & $\begin{array}{l}2 \\
2 \\
1 \\
4 \\
1\end{array}$ & $\begin{array}{l}\mathbf{A} \\
\text { A } \\
\mathbf{E} \\
\text { A } \\
\mathbf{B}\end{array}$ & $\begin{array}{l}8036.38 \\
8040.43 \\
8043.33 \\
8044.66 \\
8051.30\end{array}$ & $\begin{array}{l}1 \\
1 \\
4 \\
1 \\
2\end{array}$ & $\begin{array}{l}\mathrm{E} \\
\mathrm{E} \\
\mathrm{A} \\
\mathbf{E} \\
\mathrm{A}\end{array}$ \\
\hline $\begin{array}{l}7595.93 \\
7597.10 \\
7599.80 \\
7603.75 \\
7605.94\end{array}$ & $\begin{array}{l}2 \\
1 \\
1 \\
2 \\
2\end{array}$ & $\begin{array}{l}\mathrm{A} \\
\mathrm{B} \\
\mathrm{E} \\
\mathrm{A} \\
\mathrm{A}\end{array}$ & $\begin{array}{l}7814.33 \\
7818.85 \\
7822.00 \\
7825.24 \\
7827.90\end{array}$ & $\begin{array}{l}2 \\
3 \\
1 \\
2 \\
1\end{array}$ & $\begin{array}{l}\mathrm{A} \\
\mathbf{A} \\
\mathbf{E} \\
\mathbf{A} \\
\mathbf{A}\end{array}$ & $\begin{array}{l}8053.38 \\
8064.00 \\
8071.56 \\
8077.05 \\
8078.95\end{array}$ & $\begin{array}{l}1 \\
2 \\
2 \\
1 \\
1\end{array}$ & $\begin{array}{l}\mathbf{E} \\
\mathbf{A} \\
\mathbf{A} \\
\mathbf{D} \\
\mathbf{E}\end{array}$ \\
\hline $\begin{array}{l}7607.78 \\
7611.14 \\
7613.73 \\
7614.69 \\
7618.63\end{array}$ & $\begin{array}{l}1 \\
1 \\
1 \\
2 b, d ? \\
1\end{array}$ & $\begin{array}{l}\mathrm{E} \\
\mathrm{B} \\
\mathrm{E} \\
\mathrm{B} \\
\mathrm{B}\end{array}$ & $\begin{array}{l}7829.66 \\
7831.44 \\
7837.20 \\
7842.77 \\
7844.30\end{array}$ & $\begin{array}{l}1 \\
1 \\
2 \\
1 \\
2\end{array}$ & $\begin{array}{l}\mathrm{E} \\
\mathrm{E} \\
\mathrm{A} \\
\mathrm{A} \\
\mathrm{E} \\
\mathbf{A}\end{array}$ & $\begin{array}{l}8082.18 \\
8085.74 \\
8099.09 \\
8108.66^{\circ} \\
8118.78\end{array}$ & $\begin{array}{l}1 \\
1 \\
3 \\
2 \\
2\end{array}$ & $\begin{array}{l}\mathrm{C} \\
\mathrm{E} \\
\mathrm{A} \\
\mathrm{A} \\
\mathrm{B} \\
\mathrm{B}\end{array}$ \\
\hline
\end{tabular}


TABLE 1.-Arc Spectrum of Neodymium-Continued

\begin{tabular}{|c|c|c|c|c|c|c|c|c|}
\hline$\lambda$ I. A. & Notes & $\begin{array}{c}\text { Probable } \\
\text { error }\end{array}$ & $\lambda$ I. A. & Notes & $\begin{array}{c}\text { Probable } \\
\text { error }\end{array}$ & $\lambda$ I. A. & Notes & $\begin{array}{l}\text { Probable } \\
\text { error }\end{array}$ \\
\hline $\begin{array}{l}8120.85 \\
8122.08 \\
8131.89 \\
8133.53 \\
8141.72\end{array}$ & $\begin{array}{l}3 \\
3 \\
1 \\
1 \\
4\end{array}$ & $\begin{array}{l}\mathrm{A} \\
\mathrm{A} \\
\mathrm{B} \\
\mathrm{D} \\
\mathrm{A}\end{array}$ & $\begin{array}{l}8265.16 \\
8266.71 \\
8270.06 \\
8272.75 \\
8275.86\end{array}$ & $\begin{array}{l}1 \\
3 \\
1 \quad b \\
2 \\
1\end{array}$ & $\begin{array}{l}\text { C } \\
\text { B } \\
\text { E } \\
\text { B } \\
\text { E }\end{array}$ & $\begin{array}{l}8431.07 \\
8450.14 \\
8453.90 \\
8456.80 \\
8459.17\end{array}$ & $\begin{array}{l}1 \\
1 \text { b } \\
1 \\
3 \\
1\end{array}$ & $\begin{array}{l}\mathbf{E} \\
\mathbf{E} \\
\mathbf{E} \\
\mathbf{B} \\
\mathbf{E}\end{array}$ \\
\hline $\begin{array}{l}8143.29 \\
8150.33 \\
8158.46 \\
8161.72 \\
8164.97\end{array}$ & $\begin{array}{l}4 \\
1 \\
1 \\
1 \\
3\end{array}$ & $\begin{array}{l}\mathbf{A} \\
\mathbf{B} \\
\mathbf{B} \\
\mathbf{B} \\
\mathbf{A}\end{array}$ & $\begin{array}{l}8280.78 \\
8283.52 \\
8288.54 \\
8299.75 \\
8301.44\end{array}$ & $\begin{array}{l}1 \\
2 \\
1 \\
1 \\
1\end{array}$ & $\begin{array}{l}\mathbf{B} \\
\mathbf{B} \\
\mathbf{B} \\
\mathbf{B} \\
\mathbf{B}\end{array}$ & $\begin{array}{l}8473.13 \\
8475.32 \\
8479.98 \\
8485.80 \\
8488.36\end{array}$ & $\begin{array}{l}1 \\
1 \\
1 \\
1 \\
1\end{array}$ & $\begin{array}{l}\mathbf{E} \\
\mathbf{E} \\
\mathbf{B} \\
\mathbf{C} \\
\mathbf{E}\end{array}$ \\
\hline $\begin{array}{l}8166.79 \\
8169.78 \\
8112.49 \\
8174.48 \\
8177.24\end{array}$ & $\begin{array}{l}1 \\
2 \\
3 \\
1 \\
1\end{array}$ & $\begin{array}{l}\text { B } \\
\text { B } \\
\text { A } \\
\text { C } \\
\mathbf{E}\end{array}$ & $\begin{array}{l}8302.74 \\
8307.72 \\
8308.88 \\
8311.15 \\
8313.05\end{array}$ & $\begin{array}{l}2 \\
3 \\
1 \\
1 \\
1\end{array}$ & $\begin{array}{l}\mathbf{B} \\
\mathbf{A} \\
\mathbf{E} \\
\mathbf{B} \\
\mathbf{E}\end{array}$ & $\begin{array}{l}8514.31 \\
8522.92 \\
8527.84 \\
8530.44 \\
8533.71\end{array}$ & $\begin{array}{l}1 \\
1 \\
1 \\
1 \\
1\end{array}$ & $\begin{array}{l}\mathbf{E} \\
\mathbf{E} \\
\mathbf{B} \\
\mathbf{B} \\
\mathbf{E}\end{array}$ \\
\hline $\begin{array}{l}8179.83 \\
8182.41 \\
8185.63 \\
8194.41 \\
8198.56\end{array}$ & $\begin{array}{l}3 \\
3 \\
3 \\
1 \\
1\end{array}$ & $\begin{array}{l}\mathbf{A} \\
\mathbf{A} \\
\mathbf{A} \\
\mathbf{B} \\
\mathbf{E}\end{array}$ & $\begin{array}{l}8318.59 \\
8324.51 \\
8332.01 \\
8333.22 \\
8336.63\end{array}$ & $\begin{array}{l}1 \\
3 \\
3 \\
2 \\
1 \\
2\end{array}$ & $\begin{array}{l}\mathbf{C} \\
\mathrm{A} \\
\mathbf{B} \\
\mathbf{E} \\
\mathbf{C}\end{array}$ & $\begin{array}{l}8546.43 \\
8547.72 \\
8554.53 \\
8574.62 \\
8582.03\end{array}$ & $\begin{array}{l}1 \\
1 \\
1 \\
1 \\
1\end{array}$ & $\begin{array}{l}\mathbf{E} \\
\mathbf{E} \\
\mathbf{E} \\
\mathbf{E} \\
\mathbf{E}\end{array}$ \\
\hline $\begin{array}{l}8200.99 \\
8201.59 \\
8204.17 \\
8205.36 \\
8206.57\end{array}$ & $\begin{array}{l}1 \\
1 \text { b } \\
1 \\
3 \\
1\end{array}$ & $\begin{array}{l}\mathbf{E} \\
\mathbf{D} \\
\mathbf{C} \\
\mathbf{B} \\
\mathbf{B}\end{array}$ & $\begin{array}{l}8338.70 \\
8346.35 \\
8354.50 \\
8355.62 \\
8360.70\end{array}$ & $\begin{array}{l}1 \\
3 \\
1 \\
1 \\
1\end{array}$ & $\begin{array}{l}\mathbf{E} \\
\mathbf{A} \\
\mathbf{D} \\
\mathbf{C} \\
\mathbf{C}\end{array}$ & $\begin{array}{l}8591.43 \\
8592.15 \\
8594.84 \\
8605.48 \\
8622.83\end{array}$ & $\begin{array}{l}1 \\
1 \\
2 \\
1 \\
1\end{array}$ & $\begin{array}{l}\mathbf{E} \\
\mathbf{E} \\
\mathbf{B} \\
\mathbf{E} \\
\mathbf{E}\end{array}$ \\
\hline $\begin{array}{l}8213.17 \\
8216.97 \\
8220.27 \\
8222.28 \\
8227.36\end{array}$ & $\begin{array}{l}2 v, d ? \\
2 \\
1 \\
1 \\
2\end{array}$ & $\begin{array}{l}\mathrm{B} \\
\mathrm{B} \\
\mathrm{B} \\
\mathrm{B} \\
\mathrm{B} \\
\mathrm{B}\end{array}$ & $\begin{array}{l}8362.45 \\
8367.16 \\
8372.47 \\
8375.23 \\
8382.19\end{array}$ & $\begin{array}{l}2 \\
1 \\
1 \\
3 \\
1\end{array}$ & $\begin{array}{l}\mathbf{B} \\
\mathbf{E} \\
\mathbf{E} \\
\mathbf{B} \\
\mathbf{B}\end{array}$ & $\begin{array}{l}8625.11 \\
8643.45 \\
8656.45 \\
8667.00 \\
8677.48\end{array}$ & & $\begin{array}{l}\mathbf{E} \\
\mathbf{B} \\
\mathbf{E} \\
\mathbf{E} \\
\mathbf{B}\end{array}$ \\
\hline $\begin{array}{l}8231.47 \\
8236.41 \\
8243.08 \\
8245.42 \\
8248.77\end{array}$ & $\begin{array}{l}3 \\
1 \\
1 \\
1 \\
3\end{array}$ & $\begin{array}{l}\mathrm{A} \\
\mathrm{E} \\
\mathrm{B} \\
\mathrm{B} \\
\mathrm{B}\end{array}$ & $\begin{array}{l}8386.68 \\
8390.69 \\
8394.72 \\
8398.17 \\
8400.79\end{array}$ & $\begin{array}{l}2 \\
1 \\
2 \\
1 \\
2\end{array}$ & $\begin{array}{l}\mathrm{C} \\
\mathrm{E} \\
\mathrm{B} \\
\mathrm{B} \\
\mathrm{E} \\
\mathrm{C}\end{array}$ & $\begin{array}{l}8691.30 \\
8695.07 \\
8705.87 \\
8712.84 \\
8715.01\end{array}$ & $\begin{array}{l}1 \\
1 \\
1 \\
1 \\
1\end{array}$ & $\begin{array}{l}\mathbf{E} \\
\mathbf{E} \\
\mathbf{E} \\
\mathbf{E} \\
\mathbf{E}\end{array}$ \\
\hline $\begin{array}{l}8249.70 \\
8253.41 \\
825.21 \\
8257.97 \\
8262.80\end{array}$ & $\begin{array}{l}3 \\
1 \\
1 \\
1 \\
2\end{array}$ & $\begin{array}{l}\mathrm{B} \\
\mathrm{C} \\
\mathrm{C} \\
\mathrm{E} \\
\mathrm{B} \\
\mathrm{B}\end{array}$ & $\begin{array}{l}8403.96 \\
8408.25 \\
8409.70 \\
842.93 \\
8426.32\end{array}$ & $\begin{array}{l}1 \\
1 \mathrm{~b}, \mathrm{Cu} \\
1 \mathrm{~b} \\
1 \\
1\end{array}$ & $\begin{array}{l}\mathrm{C} \\
\mathrm{C} \\
\mathrm{E} \\
\mathrm{E} \\
\mathrm{E}\end{array}$ & $\begin{array}{l}8717.49 \\
8730.35 \\
8789.69 \\
8838.96 \\
8935.97\end{array}$ & $\begin{array}{l}1 \\
1 \\
1 \\
2 \\
1\end{array}$ & $\begin{array}{l}\mathbf{E} \\
\mathbf{E} \\
\mathbf{E} \\
\mathbf{E} \\
\mathbf{E}\end{array}$ \\
\hline
\end{tabular}

\section{SAMARIUM}

The arc spectrum of samarium, like that of neodymium, is very rich in lines, most of which are of low intensity. In Table 2 there are about I 500 wave lengths between the limits $5475 \mathrm{~A}$ and $9265 \mathrm{~A}$. Only a few faint unsymmetrical lines are suggestive of a band spectrum.

The material used for the greater portion of the work was samarium oxide supplied by the chemical laboratory of the University of Illinois. Its preparation has been described by Hopkins and Kremers. ${ }^{8} \quad$ A second series of spectrograms was made with some samarium oxalate of atomic weight grade prepared at New Hampshire College by Stewart and James. ${ }^{9}$ The arc spectra of these two preparations were identical and apparently free from the

${ }^{8}$ B. S. Sci. Papers, 17, p. 339; 1921.

9 J. Am. Chem. Soc., 39, p. 2605; 1917. 
lines of impurities, especially of other known members of the rare earth family. However, in the spectra of each of these materials appear numerous lines in agreement with some of those tabulated by Eder and attributed by him to an hypothetical element "eurosamarium." It is to be noted, however, that it is his fainter lines only that agree with wave lengths tabulated below. Almost without exception the stronger "eurosamarium" lines are not to be found in Table 2 , where the wave lengths in question are marked by the symbol "ES." Among a number of spectrograms of the rare earth preparations of C. Auer von Welsbach recently received at the Bureau of Standards from Prof. J. M. Eder, of Vienna, there were several of samarium. Some of these plates have been measured and the results are included in Table 2 .

Measurements in the arc spectrum of samarium in international units have been made by Eder, ${ }^{11}$ who, using a very pure sample of samarium sulphate prepared by Auer, derived the spectrum from $2575 \mathrm{~A}$ in the ultra-violet to $7082 \mathrm{~A}$ in the red. The agreement between his results and those given below is, in general, good, but each list contains faint lines not to be found in the other.

TABLE 2,-Arc Spectrum of Samarium

\begin{tabular}{|c|c|c|c|c|c|c|c|c|}
\hline$\lambda$ I. A. & Notes & $\begin{array}{c}\text { Probable } \\
\text { error }\end{array}$ & $\lambda$ I. A. & Notes & $\begin{array}{c}\text { Probable } \\
\text { error }\end{array}$ & $\lambda$ I. A. & Notes & $\begin{array}{c}\text { Probable } \\
\text { error }\end{array}$ \\
\hline $\begin{array}{l}\mathbf{5 4 7 5 . 2 0} \\
5478.29 \\
\mathbf{5 4 7 9 . 7 2} \\
\mathbf{5 4 8 0 . 6 6} \\
\mathbf{5 4 8 2 . 1 6}\end{array}$ & $\begin{array}{l}3 \\
4 \\
1 \\
2 \\
1\end{array}$ & $\begin{array}{l}\mathbf{B} \\
\mathrm{B} \\
\mathbf{C} \\
\mathrm{B} \\
\mathrm{C}\end{array}$ & $\begin{array}{l}5509.64 \\
5510.46 \\
5511.09 \\
5512.10 \\
5512.91\end{array}$ & $\begin{array}{l}2 \\
2 \\
3 \\
4 \\
1 \mathrm{Ca} ?\end{array}$ & $\begin{array}{l}\mathbf{B} \\
\mathbf{B} \\
\mathbf{A} \\
\mathbf{A} \\
\mathbf{B}\end{array}$ & $\begin{array}{l}5534.14 \\
5534.95 \\
5535.49 \\
5536.15 \\
5537.07\end{array}$ & $\begin{array}{l}1 \\
1 \mathrm{~b} \\
2 \mathrm{Ba} ? \\
1 \mathrm{ES} \\
4\end{array}$ & $\begin{array}{l}\text { B } \\
\mathbf{E} \\
\mathrm{E} \\
\mathrm{E} \\
\mathrm{A}\end{array}$ \\
\hline $\begin{array}{l}5484.56 \\
5485.44 \\
5486.12 \\
5487.16 \\
5488.15\end{array}$ & $\begin{array}{l}\frac{1}{5} \\
1 \\
1 \\
3 \mathrm{~d} ?\end{array}$ & $\begin{array}{l}\mathbf{B} \\
\mathbf{B} \\
\mathbf{E} \\
\mathbf{E} \\
\mathbf{B}\end{array}$ & $\begin{array}{l}5513.89 \\
5514.57 \\
5515.07 \\
5516.12 \\
5516.99\end{array}$ & $\begin{array}{l}1 \\
1 \\
1 \\
6 \\
1\end{array}$ & $\begin{array}{l}\text { B } \\
\text { E } \\
\text { E } \\
\text { A } \\
\text { B }\end{array}$ & $\begin{array}{l}5538.38 \\
5539.94 \\
5541.46 \\
5542.68\end{array}$ & $\begin{array}{l}2 \\
1 \\
3 \mathrm{~b}, \mathrm{~d}, \mathrm{~g} \\
{ }_{3} \text { coin? }\end{array}$ & $\begin{array}{l}\text { B } \\
\text { B } \\
\text { B } \\
\text { B } \\
\text { B }\end{array}$ \\
\hline $\begin{array}{l}5488.93 \\
5489.38 \\
5490.04 \\
5490.65 \\
5491.27\end{array}$ & $\begin{array}{l}2 \\
1 \\
1 \\
4 \\
1\end{array}$ & $\begin{array}{l}\mathbf{B} \\
\mathrm{C} \\
\mathrm{C} \\
\mathrm{B} \\
\mathbf{B}\end{array}$ & $\begin{array}{l}\mathbf{5 5 1 7} .58 \\
5517.98 \\
5518.31 \\
\mathbf{5 5 1 8 . 8 6} \\
5519.64\end{array}$ & $\begin{array}{l}1 \\
1 \mathrm{ES} \\
1 \\
3 \\
5\end{array}$ & $\begin{array}{l}\mathbf{E} \\
\mathbf{E} \\
\mathbf{E} \\
\mathbf{B} \\
\mathbf{B}\end{array}$ & $\begin{array}{l}5544.44 \\
5545.03 \\
5545.28 \\
5546.47\end{array}$ & $\begin{array}{l}2 \\
2 \text { ES } \\
1 \text { ES } \\
1\end{array}$ & $\begin{array}{l}\mathrm{B} \\
\mathbf{E} \\
\mathbf{E} \\
\mathbf{B}\end{array}$ \\
\hline $\begin{array}{l}5492.48 \\
5493.70 \\
5494.34 \\
5494.88 \\
5495.57\end{array}$ & $\begin{array}{l}2 \\
6 \\
3 \\
1 \\
2\end{array}$ & $\begin{array}{l}\mathbf{B} \\
\mathbf{B} \\
\mathbf{B} \\
\mathbf{B} \\
\mathbf{B}\end{array}$ & $\begin{array}{l}5520.84 \\
5521.65 \\
5522.38 \\
5523.30 \\
5524.31\end{array}$ & $\begin{array}{l}1 \\
3 \\
11, d ? \\
21, d ? \\
1\end{array}$ & $\begin{array}{l}\mathbf{E} \\
\mathbf{B} \\
\mathbf{E} \\
\mathbf{C} \\
\mathbf{E}\end{array}$ & $\begin{array}{l}5548.27 \\
5548.96 \\
5550.37 \\
5553.02\end{array}$ & $\begin{array}{l}1 \\
1 \\
4 \\
6 \mathrm{R} \\
3\end{array}$ & $\begin{array}{l}\text { B } \\
\text { A } \\
\text { A } \\
\text { B }\end{array}$ \\
\hline $\begin{array}{l}5496.74 \\
5498.21 \\
5500.88 \\
5501.66 \\
5502.69\end{array}$ & $\begin{array}{l}1 \mathrm{~b} \\
6 \\
2 \\
2 \\
1 \mathrm{~b}, \mathrm{~d} ?\end{array}$ & $\begin{array}{l}\text { B } \\
\text { A } \\
\text { B } \\
\text { B } \\
\text { B }\end{array}$ & $\begin{array}{l}5525.56 \\
5526.21 \\
5527.51 \\
5528.48 \\
5528.94\end{array}$ & $\begin{array}{l}3 \\
4 \mathrm{~b} \\
1 \mathrm{Mg} \\
1 \mathrm{Mg} \\
2 \mathrm{ES}\end{array}$ & $\begin{array}{l}\mathbf{A} \\
\mathbf{B} \\
\mathbf{E} \\
\mathbf{B} \\
\mathbf{B}\end{array}$ & $\begin{array}{l}5554.88 \\
5556.34 \\
5558.60 \\
5558.84\end{array}$ & $\begin{array}{l}1 \\
3 \text { ES } \\
2 \\
1 \text { ES } \\
1\end{array}$ & $\begin{array}{l}\mathrm{B} \\
\mathrm{B} \\
\mathrm{B} \\
\mathrm{E}\end{array}$ \\
\hline $\begin{array}{l}5503.52 \\
5504.02\end{array}$ & & $\underset{B}{B}$ & 5529.92 & $3 \underset{\text { coin? }}{\mathrm{ES}} \mathrm{g}$ & B & 5561. 39 & $\begin{array}{l}2 \\
3\end{array}$ & \\
\hline $\begin{array}{l}5507.12 \\
5509.30\end{array}$ & $\begin{array}{l}2 \text { b, ES } \\
\text { coin? } \\
1\end{array}$ & $\begin{array}{l}\text { B } \\
\text { B } \\
\text { B }\end{array}$ & $\begin{array}{l}5530.30 \\
5532.58 \\
5532.78 \\
5533.72\end{array}$ & $\begin{array}{l}1 \\
3 \\
2 \\
1\end{array}$ & $\begin{array}{l}\mathrm{E} \\
\mathbf{B} \\
\mathbf{B} \\
\mathrm{B}\end{array}$ & $\begin{array}{l}5501.39 \\
5561.78 \\
5562.33 \\
5563.09 \\
5564.24\end{array}$ & $\begin{array}{l}3 \\
1 \\
1 \\
1 \\
2\end{array}$ & $\begin{array}{l}\mathrm{A} \\
\mathrm{E} \\
\mathrm{B} \\
\mathrm{E} \\
\mathrm{B}\end{array}$ \\
\hline
\end{tabular}

${ }^{10}$ Kaiserl. Akad. der Wiss. Sitz. Wien, 126, IIa, p. 523; 1917.

11 Idem, 125, IIa, p. 883; r 916 . 
TABLE 2.-Arc Spectrum of Samarium-Continued

\begin{tabular}{|c|c|c|c|c|c|c|c|c|}
\hline$\lambda$ I. A. & Notes & $\begin{array}{c}\text { Probable } \\
\text { error }\end{array}$ & $\lambda$ I. A. & Notes & $\begin{array}{c}\text { Probable } \\
\text { error }\end{array}$ & $\lambda$ I. A. & Notes & $\begin{array}{c}\text { Probable } \\
\text { error }\end{array}$ \\
\hline $\begin{array}{l}5565.56 \\
5567.94 \\
5569.18 \\
5570.57 \\
5571.98\end{array}$ & $\begin{array}{l}2 \text { ES } \\
21, d ? \\
2 \mathrm{v}, \mathrm{d} ? \\
3 \\
1\end{array}$ & $\begin{array}{l}\mathbf{B} \\
\mathbf{B} \\
\mathbf{B} \\
\mathbf{B} \\
\mathbf{B}\end{array}$ & $\begin{array}{l}5636.68 \\
5637.30 \\
5638.65 \\
5539.88 \\
5640.27\end{array}$ & $\begin{array}{l}2 \mathrm{ES} \\
3 \\
2 \mathrm{ES} \\
\frac{1}{2}\end{array}$ & $\begin{array}{l}\mathbf{B} \\
\mathbf{A} \\
\mathbf{B} \\
\mathbf{B} \\
\mathbf{A}\end{array}$ & $\begin{array}{l}5701.12 \\
5702.23 \\
5704.10 \\
5705.72 \\
5706.77\end{array}$ & $\begin{array}{l}2 \\
1 \\
1 \mathrm{~g} \text { coin? } \\
\frac{1}{3,} \mathrm{Yt} \text { ? }\end{array}$ & $\begin{array}{l}\mathbf{E} \\
\mathbf{B} \\
\mathbf{B} \\
\mathbf{E} \\
\mathbf{A}\end{array}$ \\
\hline $\begin{array}{l}5573.42 \\
5573.94 \\
5574.32 \\
5574.86 \\
5575.61\end{array}$ & $\begin{array}{l}4 \\
1 \\
1 \\
3 \\
2\end{array}$ & $\begin{array}{l}\mathbf{A} \\
\mathbf{B} \\
\mathbf{C} \\
\mathbf{A} \\
\mathbf{B}\end{array}$ & $\begin{array}{l}5640.77 \\
5642.01 \\
5642.86 \\
5643.36 \\
5644.10\end{array}$ & $\begin{array}{l}1 \\
1 \mathrm{ES} \\
2 \mathrm{ES} \\
\frac{1}{6}\end{array}$ & $\begin{array}{l}\mathbf{B} \\
\mathbf{C} \\
\mathbf{B} \\
\mathbf{B} \\
\mathbf{A}\end{array}$ & $\begin{array}{l}5707.20 \\
5707.84 \\
5708.33 \\
5708.78 \\
5709.71\end{array}$ & $\begin{array}{l}2 \\
1 \mathrm{ES} \\
1 \mathrm{Nd} \\
3 \text { 31,d? ES } \\
3 \mathrm{ES}\end{array}$ & $\begin{array}{l}\mathbf{C} \\
\mathbf{A} \\
\mathbf{B} \\
\mathbf{B} \\
\mathbf{A}\end{array}$ \\
\hline $\begin{array}{l}5575.98 \\
5577.61 \\
5578.87 \\
5579.64 \\
5580.42\end{array}$ & $\begin{array}{l}1 \text { ES } \\
1 \\
3 \\
1 \\
1\end{array}$ & $\begin{array}{l}\mathbf{B} \\
\mathbf{C} \\
\mathbf{A} \\
\mathbf{B} \\
\mathbf{B}\end{array}$ & $\begin{array}{l}5646.00 \\
5647.31 \\
5649.00 \\
5649.59 \\
5650.37\end{array}$ & $\begin{array}{l}2 \\
4 \\
2 \\
1 \\
2\end{array}$ & $\begin{array}{l}\mathbf{B} \\
\mathbf{B} \\
\mathbf{B} \\
\mathbf{B} \\
\mathbf{B}\end{array}$ & $\begin{array}{l}5710.29 \\
5710.93 \\
5711.43 \\
5712.04 \\
5712.53\end{array}$ & $\begin{array}{l}2 \\
4 \\
3 \\
1 \\
1\end{array}$ & $\begin{array}{l}\mathbf{A} \\
\mathbf{A} \\
\mathbf{A} \\
\mathbf{B} \\
\mathbf{B}\end{array}$ \\
\hline $\begin{array}{l}5581.84 \\
5583.71 \\
5584.11 \\
5584.71 \\
5586.16\end{array}$ & $\begin{array}{l}3 \\
1 \\
21 \\
1 \\
1\end{array}$ & $\begin{array}{l}\mathbf{B} \\
\mathbf{E} \\
\mathbf{B} \\
\mathbf{E} \\
\mathbf{B}\end{array}$ & $\begin{array}{l}5651.66 \\
5652.84 \\
5655.55 \\
5556.38 \\
5657.21\end{array}$ & $\begin{array}{l}1 \\
3 \\
2 \mathrm{~b} \\
4 \\
3\end{array}$ & $\begin{array}{l}\text { B } \\
\text { B } \\
\mathbf{E} \\
\text { A } \\
\text { B }\end{array}$ & $\begin{array}{l}5713.60 \\
5714.27 \\
5715.60 \\
5716.54 \\
5717.93\end{array}$ & $\begin{array}{l}1 \\
31, \text { d? ES } \\
21, \text { ES } \\
21, \text { ES } \\
3 \text { ES }\end{array}$ & $\begin{array}{l}\mathbf{B} \\
\mathrm{B} \\
\mathbf{B} \\
\mathbf{B} \\
\mathbf{A}\end{array}$ \\
\hline $\begin{array}{l}5587.00 \\
5587.45 \\
5588.18 \\
5589.20 \\
5589.86\end{array}$ & $\begin{array}{l}2 \\
1 \\
4 \\
3 \\
3\end{array}$ & $\begin{array}{l}\text { C } \\
\text { B } \\
\text { A } \\
\text { C } \\
\AA\end{array}$ & $\begin{array}{l}5658.10 \\
5659.89 \\
5661.16 \\
5661.54 \\
5663.00\end{array}$ & $\begin{array}{l}3 \mathrm{v}, \mathrm{ES} \\
52 \\
2 \\
2 \\
2 \mathrm{ES}, \mathrm{Yt} ?\end{array}$ & $\begin{array}{l}\mathbf{B} \\
\mathbf{A} \\
\mathbf{A} \\
\mathbf{A} \\
\mathbf{A}\end{array}$ & $\begin{array}{l}5719.15 \\
5719.81 \\
5720.23 \\
5721.36 \\
5722.57\end{array}$ & $\begin{array}{l}3 \\
1 \\
3 \\
4 \\
1\end{array}$ & $\begin{array}{l}\mathbf{A} \\
\mathbf{E} \\
\mathbf{B} \\
\mathbf{A} \\
\mathbf{B}\end{array}$ \\
\hline $\begin{array}{l}5591.15 \\
5591.67 \\
5592.36 \\
5593.58 \\
5594.14\end{array}$ & $\begin{array}{l}2 \\
3 \\
31,0 \\
1 \\
1\end{array}$ & $\begin{array}{l}\mathbf{B} \\
\mathbf{A} \\
\mathbf{B} \\
\mathbf{B} \\
\mathbf{E}\end{array}$ & $\begin{array}{l}5663.89 \\
5664.73 \\
5666.04 \\
5666.70 \\
5667.41\end{array}$ & $\begin{array}{l}3 \\
2 \text { ES } \\
2 \\
2 \mathrm{~b}, \mathrm{~d} ? \\
3\end{array}$ & $\begin{array}{l}\text { A } \\
\text { A } \\
\text { B } \\
\text { C } \\
\text { B }\end{array}$ & $\begin{array}{l}5724.47 \\
5725.58 \\
5726.08 \\
5727.08 \\
5728.44\end{array}$ & $\begin{array}{l}2 \mathrm{ES} \\
2 \mathrm{ES} \\
\frac{1}{21, \mathrm{~d}} \mathrm{ES} \\
1 \mathrm{v}\end{array}$ & $\begin{array}{l}\mathbf{A} \\
\mathbf{A} \\
\mathbf{B} \\
\mathbf{B} \\
\mathbf{B}\end{array}$ \\
\hline $\begin{array}{l}5594.55 \\
5595.32 \\
5598.08 \\
5598.62 \\
5600.86\end{array}$ & $\begin{array}{l}3 \\
2 \\
1 \\
2 \\
5\end{array}$ & $\begin{array}{l}\mathbf{B} \\
\mathbf{B} \\
\mathbf{B} \\
\mathbf{B} \\
\mathbf{A}\end{array}$ & $\begin{array}{l}5667.91 \\
5668.35 \\
5668.92 \\
5669.58 \\
5670.68\end{array}$ & $\begin{array}{l}1 \\
1 \\
2 \\
2 \mathbf{b} \\
3 \mathbf{b}\end{array}$ & $\begin{array}{l}\mathrm{C} \\
\mathrm{E} \\
\mathrm{E} \\
\mathbf{B} \\
\mathbf{B}\end{array}$ & $\begin{array}{l}5729.31 \\
5730.06 \\
5731.38 \\
5732.94 \\
5733.58\end{array}$ & $\begin{array}{l}2 \mathrm{Nd?} \\
3 \\
4 \\
3 \\
3\end{array}$ & $\begin{array}{l}\mathbf{A} \\
\mathbf{B} \\
\mathbf{B} \\
\mathbf{A} \\
\mathbf{A}\end{array}$ \\
\hline $\begin{array}{l}5605.44 \\
5605.93 \\
5606.52 \\
5609.65 \\
5610.03\end{array}$ & $\begin{array}{l}\frac{1}{1} \\
2 \\
\frac{1}{2} \mathrm{ES}\end{array}$ & $\begin{array}{l}\text { B } \\
\mathbf{B} \\
\mathbf{B} \\
\mathbf{B} \\
\mathbf{B}\end{array}$ & $\begin{array}{l}5671.56 \\
5612.35 \\
5672.87 \\
567.70 \\
5674.12\end{array}$ & $\begin{array}{l}1 \\
1 \\
2 \\
1 \\
2 \mathrm{~d}\end{array}$ & $\begin{array}{l}\mathrm{C} \\
\mathrm{B} \\
\mathrm{A} \\
\mathbf{A} \\
\mathbf{B} \\
\mathbf{B}\end{array}$ & $\begin{array}{l}5734.33 \\
5735.04 \\
5736.31 \\
5736.83 \\
5738.01\end{array}$ & $\begin{array}{l}1 \\
31, \mathrm{ES} \\
\frac{1}{2} \\
4\end{array}$ & $\begin{array}{l}\mathbf{B} \\
\mathbf{B} \\
\mathbf{B} \\
\mathbf{B} \\
\mathbf{A}\end{array}$ \\
\hline $\begin{array}{l}5610.37 \\
5611.29 \\
5611.84 \\
5612.94 \\
5614.37\end{array}$ & $\begin{array}{l}1 \\
3 \mathrm{~d} \text { ? ES } \\
2 \mathrm{ES} \\
\frac{2}{2}\end{array}$ & $\begin{array}{l}\mathbf{B} \\
\mathbf{B} \\
\mathbf{B} \\
\mathbf{B} \\
\mathbf{B}\end{array}$ & $\begin{array}{l}5674.62 \\
5675.55 \\
5676.10 \\
5677.33 \\
5678.69\end{array}$ & $\begin{array}{l}1 \\
1 \\
21, \text { d? ES } \\
1 \text { lh } \\
1\end{array}$ & $\begin{array}{l}\mathbf{E} \\
\mathbf{E} \\
\mathbf{B} \\
\mathbf{B} \\
\mathbf{B}\end{array}$ & $\begin{array}{l}5738.52 \\
5739.32 \\
5741.18 \\
574.35 \\
5744.23\end{array}$ & $\begin{array}{l}1 \\
2 \\
2 \\
5 \\
2 \text { ES }\end{array}$ & $\begin{array}{l}\text { E } \\
\text { B } \\
\text { B } \\
\text { A } \\
\text { B }\end{array}$ \\
\hline $\begin{array}{l}5615.01 \\
5618.36 \\
5619.14 \\
5619.76 \\
5620.86\end{array}$ & $\begin{array}{l}1 \text { ES } \\
\frac{1}{2} \\
1 \\
1 \\
1 \text { ES }\end{array}$ & $\begin{array}{l}\mathbf{B} \\
\mathbf{C} \\
\mathbf{B} \\
\mathbf{B} \\
\mathbf{B}\end{array}$ & $\begin{array}{l}5679.13 \\
5679.72 \\
5679.99 \\
5680.90 \\
5682.40\end{array}$ & $\begin{array}{l}2 \\
3 \\
1 \\
3 \\
2 \text { ES }\end{array}$ & $\begin{array}{l}\mathrm{B} \\
\mathrm{B} \\
\mathrm{B} \\
\mathrm{E} \\
\mathrm{C} \\
\mathrm{B}\end{array}$ & $\begin{array}{l}5745.02 \\
5745.47 \\
5746.52 \\
5747.54 \\
5748.06\end{array}$ & $\begin{array}{l}2 \\
3 \\
2 \\
1 \\
3\end{array} \mathrm{~Tb} ?$ & $\begin{array}{l}\mathbf{B} \\
\mathbf{A} \\
\mathbf{A} \\
\mathbf{B} \\
\mathbf{A}\end{array}$ \\
\hline $\begin{array}{l}5621.83 \\
5622.66 \\
5622.95 \\
5624.66 \\
5624.96\end{array}$ & $\begin{array}{l}5 \\
2 \\
2 \\
2 \\
2\end{array}$ & $\begin{array}{l}\text { A } \\
\text { B } \\
\text { B } \\
\text { B } \\
\text { B }\end{array}$ & $\begin{array}{l}5683.91 \\
5684.98 \\
5685.50 \\
5686.85 \\
5687.69\end{array}$ & $\begin{array}{l}2 \\
1 \\
1 \\
5 \\
3\end{array}$ & $\begin{array}{l}\mathbf{B} \\
\mathbf{B} \\
\mathbf{E} \\
\mathbf{A} \\
\mathbf{A} \\
\mathbf{B}\end{array}$ & $\begin{array}{l}5748.45 \\
5749.46 \\
5750.73 \\
5751.42 \\
5752.16\end{array}$ & $\begin{array}{l}1 \\
1 \\
2 \\
3 \\
1 \\
1\end{array}$ & $\begin{array}{l}\mathbf{E} \\
\mathbf{B} \\
\mathbf{A} \\
\mathbf{B} \\
\mathbf{B}\end{array}$ \\
\hline $\begin{array}{l}5626.02 \\
5626.65 \\
5627.69 \\
5628.14 \\
5629.11\end{array}$ & $\begin{array}{l}5 \\
1 \\
3 \\
2 \\
1 \mathrm{~b}\end{array}$ & $\begin{array}{l}\mathbf{A} \\
\mathbf{E} \\
\mathbf{B} \\
\mathbf{B} \\
\mathbf{C}\end{array}$ & $\begin{array}{l}5688.17 \\
5688.50 \\
5689.24 \\
5690.35 \\
5690.60\end{array}$ & $\begin{array}{l}1 \\
\frac{1}{2} \mathrm{Nd} ? \\
2 \mathrm{ES} \\
1\end{array}$ & $\begin{array}{l}\mathrm{B} \\
\mathrm{B} \\
\mathrm{B} \\
\mathrm{B} \\
\mathrm{E} \\
\mathbf{E}\end{array}$ & $\begin{array}{l}5752.58 \\
5553.15 \\
5754.38 \\
575.89 \\
5756.40\end{array}$ & $\begin{array}{l}\frac{1}{2} \mathrm{ES} \\
2 \\
2 \mathrm{ES} \\
2 \mathrm{ES}\end{array}$ & $\begin{array}{l}\mathrm{B} \\
\mathbf{B} \\
\mathrm{B} \\
\mathbf{B} \\
\mathbf{A}\end{array}$ \\
\hline $\begin{array}{l}5630.81 \\
5631.73 \\
5532.03 \\
5632.35 \\
5633.03\end{array}$ & $\begin{array}{l}1 \text { ES } \\
1 \\
1 \\
1 \\
2\end{array}$ & $\begin{array}{l}\mathbf{B} \\
\mathbf{B} \\
\mathbf{B} \\
\mathbf{B} \\
\mathbf{B}\end{array}$ & $\begin{array}{l}5691.60 \\
5692.04 \\
5693.65 \\
5695.81 \\
5696.25\end{array}$ & $\begin{array}{l}2 \\
3 \\
3 \\
1 \\
3 \mathrm{Gd} ?\end{array}$ & $\begin{array}{l}\mathbf{B} \\
\mathbf{A} \\
\mathbf{A} \\
\mathbf{B} \\
\mathbf{B}\end{array}$ & $\begin{array}{l}5756.76 \\
5757.33 \\
5757.97 \\
5758.50 \\
5759.53\end{array}$ & $\begin{array}{l}1 \\
\frac{1}{3} \\
2 \\
5\end{array}$ & $\begin{array}{l}\mathbf{E} \\
\mathbf{B} \\
\mathbf{A} \\
\mathbf{B} \\
\mathbf{A}\end{array}$ \\
\hline $\begin{array}{l}5633.53 \\
5634.17 \\
5634.51 \\
5635.64 \\
5636.13\end{array}$ & $\begin{array}{l}\frac{1}{1} \mathrm{ES} \\
\frac{1}{2} \mathrm{~b} \\
1\end{array}$ & $\begin{array}{l}\mathbf{B} \\
\mathbf{B} \\
\mathbf{E} \\
\mathbf{B} \\
\mathbf{E}\end{array}$ & $\begin{array}{l}5696.75 \\
5669.77 \\
5698.40 \\
5699.58 \\
5700.25\end{array}$ & $\begin{array}{l}4 \\
1 \mathrm{~b} \\
1 \\
3 \mathrm{ES} \\
1\end{array}$ & $\begin{array}{l}\mathrm{B} \\
\mathrm{E} \\
\mathrm{E} \\
\mathrm{E} \\
\mathrm{E}\end{array}$ & $\begin{array}{l}5760.85 \\
5761.73 \\
5762.43 \\
5763.35 \\
5763.89\end{array}$ & $\begin{array}{l}3 \mathrm{~b}, \mathrm{ES} \\
2 \\
2 \\
3 \\
3\end{array}$ & $\begin{array}{l}\mathbf{B} \\
\stackrel{B}{\mathbf{B}} \\
\stackrel{\mathbf{B}}{\mathbf{B}} \\
\vec{A}\end{array}$ \\
\hline
\end{tabular}


TABLE 2.-Arc Spectrum of Samarium-Continued

\begin{tabular}{|c|c|c|c|c|c|c|c|c|}
\hline A I. A. & Notes & $\begin{array}{c}\text { Probable } \\
\text { error }\end{array}$ & $\lambda$ I. A. & Notes & $\begin{array}{c}\text { Probable } \\
\text { error }\end{array}$ & $\lambda$ I. A. & Notes & $\begin{array}{c}\text { Probable } \\
\text { error }\end{array}$ \\
\hline $\begin{array}{l}5764.31 \\
5755.01 \\
5765.89 \\
5766.46 \\
5767.12\end{array}$ & $\begin{array}{l}2 \\
3 \\
2 \\
2 \\
1\end{array}$ & $\begin{array}{l}\text { B } \\
\text { A } \\
\text { B } \\
\text { B } \\
\text { B }\end{array}$ & $\begin{array}{l}5833.40 \\
5834.14 \\
5834.84 \\
5836.37 \\
5837.42\end{array}$ & $\begin{array}{l}2 \\
\frac{1}{2} \mathrm{~b}, \mathrm{~d} \\
\frac{4}{2}\end{array}$ & $\begin{array}{l}\text { B } \\
\text { B } \\
\text { B } \\
\text { A } \\
\text { E }\end{array}$ & $\begin{array}{l}5900.69 \\
5901.38 \\
5902.35 \\
5902.61 \\
5903.04\end{array}$ & $\begin{array}{l}2 \text { ES } \\
2 \\
1 \\
3 \\
1\end{array}$ & $\begin{array}{l}\text { A } \\
\text { A } \\
\text { B } \\
\text { B } \\
\text { B }\end{array}$ \\
\hline $\begin{array}{l}5768.11 \\
5768.80 \\
5770.54 \\
5770.90 \\
5771.69\end{array}$ & $\begin{array}{l}3 \\
2 \\
1 \\
2 \mathrm{ES} \\
3 \mathrm{ES}\end{array}$ & $\begin{array}{l}\mathrm{B} \\
\mathrm{B} \\
\mathrm{B} \\
\mathrm{E} \\
\mathrm{B}\end{array}$ & $\begin{array}{l}5838.43 \\
5839.53 \\
5839.87 \\
5841.31 \\
5841.98\end{array}$ & $\begin{array}{l}2 \\
1 \\
2 \\
1 \\
2\end{array}$ & $\begin{array}{l}\mathrm{A} \\
\mathrm{E} \\
\mathrm{A} \\
\mathbf{E} \\
\mathbf{A}\end{array}$ & $\begin{array}{l}5903.53 \\
5903.97 \\
5906.05 \\
5907.91 \\
5908.47\end{array}$ & $\begin{array}{l}3 \\
1 \\
2 \\
1 \\
1\end{array}$ & $\begin{array}{l}\mathrm{A} \\
\mathrm{E} \\
\mathrm{A} \\
\mathrm{A} \\
\mathbf{E} \\
\mathbf{B}\end{array}$ \\
\hline $\begin{array}{l}5773.14 \\
5773.79 \\
5774.44 \\
5774.98 \\
5776.33\end{array}$ & $\begin{array}{l}1 \\
5 \\
1 \mathrm{~g} \text { coin? } \\
4 \mathrm{~b}\end{array}$ & $\begin{array}{l}\mathrm{E} \\
\mathrm{A} \\
\mathrm{B} \\
\mathrm{E} \\
\mathrm{B}\end{array}$ & $\begin{array}{l}5842.60 \\
5843.75 \\
5844.91 \\
5846.54 \\
5847.36\end{array}$ & & $\begin{array}{l}\text { A } \\
\text { A } \\
\text { B } \\
\text { A } \\
\text { B }\end{array}$ & $\begin{array}{l}5909.04 \\
5909.86 \\
5910.03 \\
5910.81 \\
5912.61\end{array}$ & $\begin{array}{l}3 \\
1 \mathrm{Nd} \text { ? } \\
1 \mathrm{Ce} \text { ? } \\
3 \\
3\end{array}$ & $\begin{array}{l}\mathrm{A} \\
\mathrm{E} \\
\mathrm{E} \\
\mathrm{A} \\
\mathrm{A}\end{array}$ \\
\hline $\begin{array}{l}5777.10 \\
5778.36 \\
5779.27 \\
5780.31 \\
5780.87\end{array}$ & $\begin{array}{l}2 \\
4 \mathrm{~g} \text { coin? } \\
4 \\
1 \\
1\end{array}$ & $\begin{array}{l}\mathrm{B} \\
\mathrm{A} \\
\mathrm{A} \\
\mathrm{E} \\
\mathrm{E}\end{array}$ & $\begin{array}{l}5848.67 \\
5849.71 \\
5850.94 \\
5851.98 \\
5853.07\end{array}$ & $\begin{array}{l}3 \\
2 \mathrm{ES} \\
2 \\
2 \\
2\end{array}$ & $\begin{array}{l}\mathrm{A} \\
\mathrm{A} \\
\mathrm{A} \\
\mathbf{B} \\
\mathrm{A}\end{array}$ & $\begin{array}{l}5913.56 \\
5914.90 \\
5915.56 \\
5916.37 \\
5917.94\end{array}$ & $\begin{array}{l}2 \\
2 \\
2 \\
2 \\
1 \text { ES }\end{array}$ & $\begin{array}{l}\mathrm{A} \\
\mathrm{A} \\
\mathrm{A} \\
\mathrm{A} \\
\mathrm{B}\end{array}$ \\
\hline $\begin{array}{l}5781.93 \\
5783.50 \\
5784.72 \\
5785.90\end{array}$ & $\begin{array}{l}\begin{array}{l}4 \\
3 \\
1 \\
3 \mathrm{~b}, \mathrm{~d}, \mathrm{~g} \\
5 \\
5\end{array} \text { coin? }\end{array}$ & $\begin{array}{l}\mathrm{E} \\
\mathrm{E} \\
\mathrm{E} \\
\mathrm{B}\end{array}$ & $\begin{array}{l}5853.44 \\
5854.59 \\
5855.33 \\
5855.98 \\
5857.09\end{array}$ & $\begin{array}{l}1 \\
2 \mathrm{~b} \\
1 \\
1 \\
2\end{array}$ & $\begin{array}{l}\mathrm{E} \\
\mathrm{B} \\
\mathrm{B} \\
\mathrm{B} \\
\mathrm{A}\end{array}$ & $\begin{array}{l}5919.33 \\
5920.41 \\
5921.00 \\
5921.25 \\
5921.74\end{array}$ & $\begin{array}{l}3 \\
1 \mathrm{~h} \\
3 \\
1 \\
2\end{array}$ & $\begin{array}{l}\mathrm{A} \\
\mathrm{E} \\
\mathrm{A} \\
\mathrm{E} \\
\mathrm{B}\end{array}$ \\
\hline $\begin{array}{l}5787.55 \\
578.39 \\
5789.35 \\
5790.36 \\
5791.00\end{array}$ & $\begin{array}{l}2 \\
4 \\
1 \\
2 \\
3 \mathrm{ES}\end{array}$ & $\begin{array}{l}\text { A } \\
\text { A } \\
\text { B } \\
\text { B } \\
\text { B }\end{array}$ & $\begin{array}{l}5858.09 \\
5859.23 \\
5859.78 \\
5860.36 \\
5860.82\end{array}$ & $\begin{array}{l}2 \\
2 \\
1 \\
4 \mathrm{~d} \text { ? } \\
2\end{array}$ & $\begin{array}{l}\text { B } \\
\text { B } \\
\text { E } \\
\text { A } \\
\text { B }\end{array}$ & $\begin{array}{l}5922.44 \\
5923.33 \\
5924.18 \\
5924.66 \\
5925.92\end{array}$ & $\begin{array}{l}21, \text { ES } \\
3 \\
2 \\
2 \\
1\end{array}$ & $\begin{array}{l}\mathrm{B} \\
\mathrm{A} \\
\mathrm{A} \\
\mathrm{A} \\
\mathrm{B}\end{array}$ \\
\hline $\begin{array}{l}5791.77 \\
5792.76 \\
5793.29 \\
5794.04 \\
5794.22\end{array}$ & $\begin{array}{l}2 \\
1 \\
3 \\
1 \\
3\end{array}$ & $\begin{array}{l}\mathrm{B} \\
\mathrm{E} \\
\mathrm{B} \\
\mathrm{E} \\
\mathrm{E} \\
\mathrm{B}\end{array}$ & $\begin{array}{l}5861.41 \\
5862.50 \\
5863.85 \\
5864.47 \\
5866.13\end{array}$ & $\begin{array}{l}1 \\
1 \\
1 \\
2 \\
1\end{array}$ & $\begin{array}{l}\mathbf{E} \\
\mathbf{E} \\
\mathbf{E} \\
\mathrm{A} \\
\mathrm{E}\end{array}$ & $\begin{array}{l}5926.44 \\
5927.83 \\
5928.35 \\
5929.19 \\
5930.89\end{array}$ & $\begin{array}{l}2 \\
2 \\
1 \\
2 \\
1 \mathrm{v}\end{array}$ & $\begin{array}{l}\mathrm{A} \\
\mathrm{A} \\
\mathrm{A} \\
\mathrm{B} \\
\mathrm{B}\end{array}$ \\
\hline $\begin{array}{l}5795.29 \\
5797.31 \\
5797.86 \\
5798.64 \\
5799.50\end{array}$ & $\begin{array}{l}2 \\
1 \\
2 \\
1 \\
1\end{array}$ & $\begin{array}{l}\mathrm{B} \\
\mathrm{B} \\
\mathrm{C} \\
\mathrm{C} \\
\mathrm{E}\end{array}$ & $\begin{array}{l}5857.38 \\
5867.77 \\
5868.62 \\
5869.93 \\
5870.31\end{array}$ & $\begin{array}{l}1 \\
4 \\
4 \\
1 \\
1\end{array}$ & $\begin{array}{l}\mathbf{E} \\
\mathrm{A} \\
\mathrm{A} \\
\mathrm{E} \\
\mathrm{E}\end{array}$ & $\begin{array}{l}5931.52 \\
5932.20 \\
5932.90 \\
5933.94 \\
5935.05\end{array}$ & $\begin{array}{l}1 \text { ES } \\
3 \\
2 \\
1 \\
1 \text { ES }\end{array}$ & $\begin{array}{l}\text { B } \\
\text { B } \\
\text { A } \\
\text { B } \\
\text { B }\end{array}$ \\
\hline $\begin{array}{l}5800.51 \\
5801.24 \\
5801.66 \\
5802.86 \\
5803.94\end{array}$ & $\begin{array}{l}4 \\
3 \\
2 \\
5 \\
1\end{array}$ & $\begin{array}{l}\mathrm{A} \\
\mathrm{A} \\
\mathrm{A} \\
\mathrm{A} \\
\mathrm{E}\end{array}$ & $\begin{array}{l}5871.07 \\
5872.29 \\
5873.02 \\
5874.22 \\
5875.09\end{array}$ & $\begin{array}{l}3 \\
1 \\
1 \\
4 \\
2\end{array}$ & $\begin{array}{l}\mathrm{A} \\
\mathrm{E} \\
\mathrm{E} \\
\mathrm{E} \\
\mathrm{A} \\
\mathrm{A}\end{array}$ & $\begin{array}{l}5936.12 \\
5937.76 \\
5938.90 \\
5939.92 \\
5940.69\end{array}$ & $\begin{array}{l}2 \\
1 \\
4 \\
1 \mathrm{~b} \\
1\end{array}$ & $\begin{array}{l}\mathrm{A} \\
\mathrm{B} \\
\mathrm{A} \\
\mathrm{B} \\
\mathrm{C}\end{array}$ \\
\hline $\begin{array}{l}5804.70 \\
5805.69 \\
5806.25 \\
5806.77 \\
5810.33\end{array}$ & $\begin{array}{l}2 \\
1 \mathrm{Ba} \\
\frac{1}{3} \\
2 \mathrm{ES}\end{array}$ & $\begin{array}{l}\mathrm{A} \\
\mathrm{E} \\
\mathrm{B} \\
\mathrm{A} \\
\mathrm{A} \\
\mathrm{B}\end{array}$ & $\begin{array}{l}5875.92 \\
5876.82 \\
5878.11 \\
5878.42 \\
5879.36\end{array}$ & $\begin{array}{l}2 \\
2 \\
3 \\
1 \\
2 \mathrm{vS} \\
2 \mathrm{~d} \text { ? }\end{array}$ & $\begin{array}{l}\text { A } \\
\text { A } \\
\text { A } \\
\text { E } \\
\text { B }\end{array}$ & $\begin{array}{l}5941.43 \\
5942.30 \\
5943.00 \\
5944.72 \\
5946.33\end{array}$ & $\begin{array}{l}3 \\
3 \\
2 \\
1 \mathrm{v} \\
2\end{array}$ & $\begin{array}{l}\mathbf{B} \\
\mathbf{A} \\
\mathbf{A} \\
\mathbf{B} \\
\mathbf{A}\end{array}$ \\
\hline $\begin{array}{l}5811.44 \\
5812.66 \\
5813.08 \\
5813.84 \\
5814.88\end{array}$ & $\begin{array}{l}2 \mathrm{~b} \\
3 \\
2 \\
1 \\
5\end{array}$ & $\begin{array}{l}\mathrm{B} \\
\mathrm{A} \\
\mathbf{B} \\
\mathrm{B} \\
\mathrm{E} \\
\mathrm{A}\end{array}$ & $\begin{array}{l}5880.51 \\
5881.64 \\
5382.51 \\
5883.67 \\
5885.47\end{array}$ & $\begin{array}{l}1 \mathrm{ES} \\
2 \mathrm{~b}, \mathrm{~d} ? \\
2 \\
2 \mathrm{ES} \\
1\end{array}$ & $\begin{array}{l}\mathbf{B} \\
\mathbf{B} \\
\mathbf{A} \\
\mathbf{A} \\
\mathbf{B}\end{array}$ & $\begin{array}{l}5947.43 \\
5950.14 \\
5951.95 \\
5952.37 \\
5955.82\end{array}$ & $\begin{array}{l}2 \text { ES } \\
1 \text { V } \\
1 \\
1 \\
3\end{array}$ & $\begin{array}{l}\text { B } \\
\text { B } \\
\text { B } \\
\text { B } \\
\text { A }\end{array}$ \\
\hline $\begin{array}{l}5816.34 \\
5817.49 \\
5819.51 \\
5820.68 \\
5823.18\end{array}$ & $\begin{array}{l}3 \\
2 \\
2 \\
2 \text { b, d } \\
2 \text { ES }\end{array}$ & $\begin{array}{l}\text { A } \\
\text { B } \\
\text { B } \\
\text { A } \\
\text { B }\end{array}$ & $\begin{array}{l}5835.04 \\
5886.47 \\
5887.27 \\
5388.01 \\
5889.77\end{array}$ & $\begin{array}{l}1 \\
1 \\
2 \\
31, d ? \\
3\end{array}$ & $\begin{array}{l}\text { B } \\
\text { B } \\
\text { A } \\
\text { C } \\
\text { C }\end{array}$ & $\begin{array}{l}5956.79 \\
5957.52 \\
5958.21 \\
5960.09 \\
5963.92\end{array}$ & $\begin{array}{l}2 \\
2 \\
1 \\
2 \\
1\end{array}$ & $\begin{array}{l}\mathrm{A} \\
\mathrm{A} \\
\mathrm{A} \\
\mathrm{A} \\
\mathrm{A}\end{array}$ \\
\hline $\begin{array}{l}5824.00 \\
5825.64 \\
5826.30 \\
5827.10 \\
5827.62\end{array}$ & $\begin{array}{l}3 \mathrm{~b} \\
3 \\
1 \mathrm{Ba} ? \\
\frac{1}{2}\end{array}$ & $\begin{array}{l}\text { A } \\
\text { A } \\
\text { B } \\
\text { B } \\
\text { B }\end{array}$ & $\begin{array}{l}5890.61 \\
5891.42 \\
5892.22 \\
5894.73 \\
5895.18\end{array}$ & $\begin{array}{l}2 \\
2 \\
3 \mathrm{~b} \\
1 \\
2 \mathrm{I}\end{array}$ & $\begin{array}{l}\mathbf{A} \\
\mathbf{A} \\
\mathbf{B} \\
\mathbf{B} \\
\mathbf{A}\end{array}$ & $\begin{array}{l}5965.71 \\
5966.36 \\
5967.06 \\
5967.74 \\
5968.82\end{array}$ & $\begin{array}{l}4 \\
1 \\
2 \\
1 \\
3\end{array}$ & $\begin{array}{l}\text { A } \\
\text { B } \\
\text { B } \\
\text { B } \\
\text { A }\end{array}$ \\
\hline $\begin{array}{l}5828.53 \\
5830.50 \\
5831.02 \\
5831.74 \\
5832.42\end{array}$ & $\begin{array}{l}1 \\
3 \\
3 \\
3 \\
2\end{array}$ & $\begin{array}{l}\text { C } \\
\text { A } \\
\text { A } \\
\text { A } \\
\text { B }\end{array}$ & $\begin{array}{l}5896.31 \\
5896.75 \\
5897.39 \\
5898.96 \\
5899.73\end{array}$ & $\begin{array}{l}2 \\
1 \\
3 \\
3 \\
1\end{array}$ & $\begin{array}{l}\text { B } \\
\text { B } \\
\text { A } \\
\text { A } \\
\text { B }\end{array}$ & $\begin{array}{l}5969.50 \\
5970.81 \\
5971.47 \\
5972.10 \\
5972.55\end{array}$ & $\begin{array}{l}2 \\
2 \\
1 \\
1 \\
1\end{array}$ & $\begin{array}{l}\mathrm{A} \\
\mathrm{B} \\
\mathrm{B} \\
\mathrm{A} \\
\mathrm{A}\end{array}$ \\
\hline
\end{tabular}


TABLE 2.-Arc Spectrum of Samarium-Continued

\begin{tabular}{|c|c|c|c|c|c|c|c|c|}
\hline$\lambda$ I. A. & Notes & $\begin{array}{c}\text { Probable } \\
\text { error }\end{array}$ & $\lambda$ I. A. & Notes & $\underset{\text { error }}{\text { Probable }}$ & $\lambda$ I. A. & Notes & $\begin{array}{c}\text { Probable } \\
\text { error }\end{array}$ \\
\hline $\begin{array}{l}5973.03 \\
5975.54 \\
5976.43 \\
5978.02 \\
5979.38\end{array}$ & $\begin{array}{l}1 \\
1 \\
1 \\
\frac{1}{2} \mathrm{ES} \\
2\end{array}$ & $\begin{array}{l}\mathbf{B} \\
\mathbf{E} \\
\mathbf{B} \\
\mathbf{A} \\
\mathbf{A}\end{array}$ & $\begin{array}{l}6061.24 \\
6063.67 \\
6066.64 \\
6067.38 \\
6067.78\end{array}$ & $\begin{array}{l}2 \mathrm{ES} \\
1 \mathrm{ES} \\
1 \\
2 \\
2\end{array}$ & $\begin{array}{l}\mathrm{A} \\
\mathrm{B} \\
\mathrm{B} \\
\mathrm{A} \\
\mathrm{A} \\
\mathrm{A}\end{array}$ & $\begin{array}{l}6160.42 \\
6163.94 \\
6164.51 \\
6164.98 \\
6165.85\end{array}$ & $\begin{array}{l}3 \\
\frac{1}{2} \text { ES } \\
\frac{1}{2}\end{array}$ & $\begin{array}{l}\mathrm{A} \\
\mathrm{B} \\
\mathrm{A} \\
\mathbf{E} \\
\mathrm{A}\end{array}$ \\
\hline $\begin{array}{l}5980.46 \\
5981.98 \\
5983.13 \\
5983.75 \\
5985.48\end{array}$ & $\begin{array}{l}2 \mathrm{~b} \\
2 \\
1 \\
2 \mathrm{~b} \\
1\end{array}$ & $\begin{array}{l}\mathbf{B} \\
\mathbf{A} \\
\mathbf{C} \\
\mathbf{B} \\
\mathbf{B}\end{array}$ & $\begin{array}{l}6068.27 \\
6077.06 \\
6070.56 \\
6074.96 \\
6077.83\end{array}$ & $\begin{array}{l}1 \\
3 \\
1 \\
2 \\
1\end{array}$ & $\begin{array}{l}\mathrm{A} \\
\mathrm{A} \\
\mathrm{B} \\
\mathrm{B} \\
\mathrm{B}\end{array}$ & $\begin{array}{l}6166.76 \\
6168.33 \\
6169.85 \\
6173.10 \\
6173.94\end{array}$ & $\begin{array}{l}2 \text { ES } \\
2 \\
1 \\
2 \\
2 \text { ES }\end{array}$ & $\begin{array}{l}\mathbf{B} \\
\mathbf{A} \\
\mathbf{E} \\
\mathbf{B} \\
\mathbf{A}\end{array}$ \\
\hline $\begin{array}{l}5989.69 \\
5991.69 \\
5993.85 \\
5994.64 \\
5995.14\end{array}$ & $\begin{array}{l}2 \\
2 \mathrm{~b} \\
2 \\
2 \\
2\end{array}$ & $\begin{array}{l}\mathrm{A} \\
\mathrm{A} \\
\mathrm{A} \\
\mathrm{A} \\
\mathrm{A}\end{array}$ & $\begin{array}{l}6080.02 \\
6080.53 \\
6081.99 \\
6088.66 \\
6086.09\end{array}$ & $\begin{array}{l}l_{1}^{1} \mathrm{ES} \\
\frac{1}{2} \mathrm{ES} \\
1\end{array}$ & $\begin{array}{l}\mathrm{B} \\
\mathrm{C} \\
\mathrm{B} \\
\mathrm{A} \\
\mathrm{B}\end{array}$ & $\begin{array}{l}6174.45 \\
6174.96 \\
6176.40 \\
6177.45 \\
6178.65\end{array}$ & $\begin{array}{l}1 \\
1 \\
1 \text { ES } \\
1 \text { Nd? }\end{array}$ & $\begin{array}{l}\mathrm{A} \\
\mathrm{A} \\
\mathrm{B} \\
\mathrm{A} \\
\mathrm{E}\end{array}$ \\
\hline $\begin{array}{l}5996.06 \\
5996.83 \\
5997.32 \\
5998.08 \\
6001.09\end{array}$ & $\begin{array}{l}2 \\
1 \mathrm{v} \\
2 \\
1 \\
1\end{array}$ & $\begin{array}{l}\text { A } \\
\text { B } \\
\text { A } \\
\text { B } \\
\text { B }\end{array}$ & $\begin{array}{l}6087.67 \\
6088.09 \\
6088.63 \\
6090.47 \\
6091.36\end{array}$ & $\begin{array}{l}1 \\
1 \text { ES } \\
2 \\
1 \\
3\end{array}$ & $\begin{array}{l}\mathbf{B} \\
\mathbf{B} \\
\mathbf{A} \\
\mathbf{B} \\
\mathbf{A}\end{array}$ & $\begin{array}{l}6179.40 \\
6179.82 \\
6181.05 \\
6181.88 \\
6182.89\end{array}$ & $\begin{array}{l}2 \\
2 \\
2 \\
2 \\
2\end{array}$ & $\begin{array}{l}\mathbf{A} \\
\mathbf{B} \\
\mathbf{A} \\
\mathbf{E} \\
\mathbf{A}\end{array}$ \\
\hline $\begin{array}{l}6001.70 \\
6002.84 \\
6004.20 \\
6005.14 \\
6006.10\end{array}$ & $\begin{array}{l}2 \\
2 \\
2 \\
11 \\
1\end{array}$ & $\begin{array}{l}\mathbf{B} \\
\mathbf{B} \\
\mathbf{A} \\
\mathbf{B} \\
\mathbf{E}\end{array}$ & $\begin{array}{l}6091.94 \\
6092.92 \\
6093.69 \\
6094.10 \\
6095.25\end{array}$ & $\begin{array}{l}1 \\
1 \\
1 \\
1 \\
2\end{array}$ & $\begin{array}{l}\text { B } \\
\text { B } \\
\text { B } \\
\text { B } \\
\text { B }\end{array}$ & $\begin{array}{l}6186.24 \\
6186.85 \\
6187.42 \\
6188.00 \\
6189.80\end{array}$ & $\begin{array}{l}1 \\
1 \\
1 \\
2 \\
1 \text { b, ES }\end{array}$ & $\begin{array}{l}\mathbf{C} \\
\mathbf{C} \\
\mathbf{E} \\
\mathbf{A} \\
\mathbf{A}\end{array}$ \\
\hline $\begin{array}{l}6007.94 \\
6009.87 \\
6010.26 \\
6010.76 \\
6011.27\end{array}$ & $\begin{array}{l}2 \text { b, ES } \\
2 \\
1 \\
1 \\
2\end{array}$ & $\begin{array}{l}\mathbf{B} \\
\mathbf{B} \\
\mathbf{B} \\
\mathbf{B} \\
\mathbf{A}\end{array}$ & $\begin{array}{l}6096.77 \\
6099.06 \\
6099.90 \\
6101.40 \\
6101.96\end{array}$ & $\begin{array}{l}2 \text { ES } \\
2 \\
2 \\
1 \\
3\end{array}$ & $\begin{array}{l}\mathbf{A} \\
\mathbf{A} \\
\mathbf{A} \\
\mathbf{B} \\
\mathbf{A}\end{array}$ & $\begin{array}{l}6191.24 \\
6192.62 \\
6193.07 \\
6194.37 \\
6195.14\end{array}$ & $\begin{array}{l}1 \mathrm{I}, \mathrm{ES} \\
2 \\
1 \\
2 \\
1\end{array}$ & $\begin{array}{l}\mathbf{A} \\
\mathbf{A} \\
\mathbf{E} \\
\mathbf{B} \\
\mathbf{E}\end{array}$ \\
\hline $\begin{array}{l}6012.81 \\
6013.40 \\
6013.83 \\
6016.10 \\
6016.78\end{array}$ & $\begin{array}{l}\frac{1}{2} \mathrm{C} \\
1 \\
\frac{1}{2}\end{array}$ & $\begin{array}{l}\mathbf{B} \\
\mathbf{B} \\
\mathbf{B} \\
\mathbf{B} \\
\mathbf{B}\end{array}$ & $\begin{array}{l}6103.32 \\
610.82 \\
6105.36 \\
6107.13 \\
6109.23\end{array}$ & $\begin{array}{l}3 \\
2 \\
1 \\
1 \\
1\end{array}$ & $\begin{array}{l}\mathbf{A} \\
\mathbf{A} \\
\mathbf{B} \\
\mathbf{B} \\
\mathbf{B}\end{array}$ & $\begin{array}{l}6196.08 \\
6196.57 \\
6197.37 \\
6198.14 \\
6199.41\end{array}$ & ${ }_{1}^{1} \mathrm{~b}, \mathrm{~d}$ ?ES & $\begin{array}{l}\mathrm{E} \\
\mathrm{E} \\
\mathrm{E} \\
\mathrm{A} \\
\mathrm{A} \\
\mathrm{B}\end{array}$ \\
\hline $\begin{array}{l}6017.39 \\
6019.17 \\
6019.93 \\
6021.26 \\
6021.75\end{array}$ & $\begin{array}{l}3 \\
1 \\
\frac{1}{2} \mathrm{Yt} \\
2\end{array}$ & $\begin{array}{l}\mathbf{A} \\
\mathbf{E} \\
\mathbf{C} \\
\mathrm{B} \\
\mathbf{B}\end{array}$ & $\begin{array}{l}6110.19 \\
6110.66 \\
6112.94 \\
6114.58 \\
6115.70\end{array}$ & $\begin{array}{l}2 \\
3 \\
2 \\
3 \\
1\end{array}$ & $\begin{array}{l}\mathrm{B} \\
\mathrm{A} \\
\mathrm{A} \\
\mathrm{A} \\
\mathbf{E}\end{array}$ & $\begin{array}{l}6200.07 \\
6201.11 \\
6202.44 \\
6203.89 \\
6205.78\end{array}$ & $\begin{array}{l}1 \text { ES } \\
1 \\
1 \\
2 \\
1\end{array}$ & $\begin{array}{l}\mathbf{B} \\
\mathbf{B} \\
\mathbf{E} \\
\mathbf{A} \\
\mathbf{B}\end{array}$ \\
\hline $\begin{array}{l}6023.17 \\
6023.65 \\
6024.44 \\
6027.14 \\
6027.54\end{array}$ & $\begin{array}{l}1 \\
2 \\
1 \mathrm{~b}, \mathrm{~d} ? \\
2 \\
2\end{array}$ & $\begin{array}{l}\mathbf{B} \\
\mathbf{B} \\
\mathbf{B} \\
\mathbf{A} \\
\mathbf{A}\end{array}$ & $\begin{array}{l}6117.78 \\
6120.26 \\
6121.81 \\
612.73 \\
6123.60\end{array}$ & $\begin{array}{l}2 v \\
1 \\
2 \\
2 \\
2\end{array}$ & $\begin{array}{l}\mathbf{B} \\
\mathbf{E} \\
\mathbf{B} \\
\mathbf{A} \\
\mathbf{A}\end{array}$ & $\begin{array}{l}6206.22 \\
6206.83 \\
6207.05 \\
6208.98 \\
6210.42\end{array}$ & $\begin{array}{l}\frac{1}{2} \mathrm{~b}, \mathrm{~d} \text { ? } \\
\frac{1}{1}\end{array}$ & $\begin{array}{l}\mathrm{B} \\
\mathrm{A} \\
\mathrm{A} \\
\mathrm{B} \\
\mathrm{B} \\
\mathrm{A}\end{array}$ \\
\hline $\begin{array}{l}6028.67 \\
6029.80 \\
6032.45 \\
6033.23 \\
6033.83\end{array}$ & $\begin{array}{l}2 \\
1 \\
1 \\
1 \\
1 \text { ES }\end{array}$ & $\begin{array}{l}\text { B } \\
\text { A } \\
\text { A } \\
\text { A } \\
\mathbf{A}\end{array}$ & $\begin{array}{l}6124.88 \\
6125.83 \\
6126.98 \\
6128.76 \\
6129.85\end{array}$ & $\begin{array}{l}2 \\
1 \\
2 \\
2 \\
2\end{array}$ & $\begin{array}{l}\text { B } \\
\text { B } \\
\text { A } \\
\text { A } \\
\text { A }\end{array}$ & $\begin{array}{l}6212.64 \\
6213.81 \\
6216.44 \\
6217.08 \\
6218.22\end{array}$ & $\begin{array}{l}11 \\
1 \\
1 \\
2 \\
1\end{array}$ & $\begin{array}{l}\mathbf{E} \\
\mathbf{A} \\
\mathbf{E} \\
\mathbf{A} \\
\mathrm{A}\end{array}$ \\
\hline $\begin{array}{l}6035.54 \\
6036.42 \\
6041.40 \\
6042.76 \\
6043.33\end{array}$ & $\begin{array}{l}1 \mathrm{ES} \\
11 \mathrm{ES} \\
3 \\
2 \mathrm{~b}, \mathrm{v}, \mathrm{ES}\end{array}$ & $\begin{array}{l}\mathbf{A} \\
\mathbf{B} \\
\mathbf{A} \\
\mathbf{B} \\
\mathbf{B}\end{array}$ & $\begin{array}{l}6130.56 \\
6131.15 \\
6133.16 \\
6138.01 \\
6138.47\end{array}$ & $\begin{array}{l}2 \\
2 \\
2 \\
2 \\
1\end{array}$ & $\begin{array}{l}\mathrm{A} \\
\mathrm{A} \\
\mathrm{A} \\
\mathrm{A} \\
\mathrm{A}\end{array}$ & $\begin{array}{l}6219.17 \\
6220.83 \\
6221.47 \\
6222.66 \\
6223.62\end{array}$ & & $\begin{array}{l}\mathbf{A} \\
\mathbf{B} \\
\mathbf{E} \\
\mathbf{A} \\
\mathbf{B}\end{array}$ \\
\hline $\begin{array}{l}6044.99 \\
6045.42 \\
6046.40 \\
6047.42 \\
6049.20\end{array}$ & $\begin{array}{l}3 \\
3 \\
1 \\
2 \\
1 \\
1\end{array}$ & $\begin{array}{l}\mathbf{A} \\
\mathbf{A} \\
\mathbf{B} \\
\mathbf{B} \\
\mathbf{B}\end{array}$ & $\begin{array}{l}6139.31 \\
6140.56 \\
6143.13 \\
6143.61 \\
6143.97\end{array}$ & $\begin{array}{l}2 \\
3 \\
2 \\
1 \\
2\end{array}$ & $\begin{array}{l}\mathbf{A} \\
\mathbf{A} \\
\mathbf{A} \\
\mathbf{A} \\
\mathbf{A}\end{array}$ & $\begin{array}{l}6224.61 \\
6225.19 \\
6225.57 \\
6226.71 \\
6228.26\end{array}$ & $\begin{array}{l}1 \\
1 \\
1 \\
1 \\
1\end{array}$ & $\begin{array}{l}\mathbf{E} \\
\mathbf{B} \\
\mathbf{A} \\
\mathbf{A} \\
\mathbf{B}\end{array}$ \\
\hline $\begin{array}{l}6049.81 \\
6050.28 \\
6052.07 \\
6053.10 \\
6056.50\end{array}$ & $\begin{array}{l}2 \mathrm{~b}, 1 \\
2 \mathrm{~b} \\
1 \\
3 \\
3\end{array}$ & $\begin{array}{l}\mathrm{B} \\
\mathrm{E} \\
\mathrm{B} \\
\mathrm{B} \\
\mathrm{A}\end{array}$ & $\begin{array}{l}6144.76 \\
6146.52 \\
6147.37 \\
6148.23 \\
6149.10\end{array}$ & $\begin{array}{l}1 \mathrm{~h}, 1 \\
2 \\
1 \\
1 \\
3\end{array}$ & $\begin{array}{l}\mathbf{E} \\
\mathbf{A} \\
\mathbf{E} \\
\mathbf{A} \\
\mathbf{A}\end{array}$ & $\begin{array}{l}6231.66 \\
6232.74 \\
6233.70 \\
6234.20 \\
6235.29\end{array}$ & $\begin{array}{l}1 \\
1 \text { b } \\
1 \\
1 \\
1\end{array}$ & $\begin{array}{l}\mathbf{B} \\
\mathbf{E} \\
\mathbf{E} \\
\mathbf{B} \\
\mathbf{B}\end{array}$ \\
\hline $\begin{array}{l}6057.24 \\
6057.67 \\
6058.40 \\
6059.86 \\
6050.73\end{array}$ & $\begin{array}{l}2 \\
2 \\
1 \\
2 \mathrm{ES} \\
2 \mathrm{ES}\end{array}$ & $\begin{array}{l}\mathrm{B} \\
\mathrm{B} \\
\mathrm{B} \\
\mathrm{A} \\
\mathrm{A}\end{array}$ & $\begin{array}{l}6149.90 \\
6150.90 \\
6154.83 \\
6157.55 \\
6159.48\end{array}$ & $\begin{array}{l}1 \\
1 \mathrm{ES} \\
1 \mathrm{~h} \\
2 \mathrm{ES} \\
4\end{array}$ & $\begin{array}{l}\mathbf{B} \\
\mathbf{B} \\
\mathbf{E} \\
\mathbf{A} \\
\mathbf{A}\end{array}$ & $\begin{array}{l}6236.12 \\
6237.66 \\
6238.30 \\
6238.89 \\
6240.45\end{array}$ & $\begin{array}{l}1 \\
3 \\
2 \\
1 \\
1\end{array}$ & $\begin{array}{l}\mathbf{B} \\
\mathbf{A} \\
\mathbf{A} \\
\mathbf{B} \\
\mathbf{A} \\
\mathbf{A}\end{array}$ \\
\hline
\end{tabular}


TABLE 2.-Arc Spectrum of Samarium-Continued

\begin{tabular}{|c|c|c|c|c|c|c|c|c|}
\hline X I. A. & Notes & $\begin{array}{c}\text { Probable } \\
\text { error }\end{array}$ & $\lambda$ I A. & Notes & $\begin{array}{c}\text { Probable } \\
\text { error }\end{array}$ & $\lambda$ I. A. & Notes & $\begin{array}{c}\text { Probable } \\
\text { error }\end{array}$ \\
\hline $\begin{array}{l}6241.54 \\
6242.38 \\
6244.21 \\
6246.76 \\
6248.09\end{array}$ & $\begin{array}{l}1 \\
1 \\
2 \\
3 \\
2\end{array}$ & $\begin{array}{l}\mathbf{A} \\
\mathbf{A} \\
\mathbf{A} \\
\mathbf{A} \\
\mathbf{A}\end{array}$ & $\begin{array}{l}6352.97 \\
6353.47 \\
6355.34 \\
6357.27 \\
6363.12\end{array}$ & $\begin{array}{l}1 \\
3 \\
2 \\
3 \\
1\end{array}$ & $\begin{array}{l}\mathbf{A} \\
\mathbf{A} \\
\stackrel{A}{A} \\
\mathbf{E}\end{array}$ & $\begin{array}{l}6474.25 \\
6475.47 \\
6476.43 \\
6477.02 \\
6478.22\end{array}$ & $\begin{array}{l}3 \\
1 \\
1 \\
2 \\
1\end{array}$ & $\begin{array}{l}\mathbf{E} \\
\mathbf{E} \\
\mathbf{B} \\
\mathbf{B} \\
\mathbf{E}\end{array}$ \\
\hline $\begin{array}{l}6251.65 \\
6256.02 \\
6256.66 \\
6257.91 \\
6259.65\end{array}$ & $\begin{array}{l}1 \\
1 \\
1 \\
5 \\
1 \\
1\end{array}$ & $\begin{array}{l}\mathrm{A} \\
\mathrm{A} \\
\mathrm{A} \\
\mathrm{A} \\
\mathrm{B}\end{array}$ & $\begin{array}{l}6366.17 \\
6367.39 \\
6368.28 \\
6371.00 \\
6371.89\end{array}$ & $\begin{array}{l}1 \\
2 \\
3 \\
2 \\
2\end{array}$ & $\begin{array}{l}\mathbf{E} \\
\mathbf{A} \\
\mathbf{A} \\
\mathbf{A} \\
\mathbf{A}\end{array}$ & $\begin{array}{l}6479.17 \\
6483.35 \\
6484.49 \\
6487.62 \\
6488.56\end{array}$ & $\begin{array}{l}1 \\
11 \\
4 \\
4 \\
1\end{array}$ & $\begin{array}{l}\mathbf{E} \\
\mathbf{E} \\
\mathbf{A} \\
\mathbf{A} \\
\mathbf{E}\end{array}$ \\
\hline $\begin{array}{l}6260.09 \\
6262.50 \\
6264.15 \\
6265.59 \\
6267.28\end{array}$ & $\begin{array}{ll}1 & \\
1 & v \\
1 & b \\
1 & \\
4 & \end{array}$ & $\begin{array}{l}\text { B } \\
\text { B } \\
\text { B } \\
\mathbf{E} \\
\mathbf{A}\end{array}$ & $\begin{array}{l}6377.18 \\
6378.01 \\
6380.04 \\
6380.67 \\
6381.60\end{array}$ & $\begin{array}{l}1 \text { b } \\
1 \\
2 \\
2 \\
1\end{array}$ & $\begin{array}{l}\mathbf{E} \\
\mathbf{E} \\
\mathbf{A} \\
\mathbf{A} \\
\mathbf{E}\end{array}$ & $\begin{array}{l}6490.82 \\
6492.02 \\
6493.14 \\
6496.05 \\
6497.83\end{array}$ & $\begin{array}{l}4 \\
1 \\
1 \\
1 \\
2\end{array}$ & $\begin{array}{l}\mathbf{A} \\
\mathbf{E} \\
\mathbf{E} \\
\mathbf{A} \\
\mathbf{B}\end{array}$ \\
\hline $\begin{array}{l}6270.01 \\
6271.43 \\
6272.37 \\
6273.32 \\
6273.69\end{array}$ & $\begin{array}{l}1 \\
1 \\
1 \\
1 \\
1\end{array}$ & $\begin{array}{l}\mathbf{A} \\
\mathbf{A} \\
\mathbf{B} \\
\mathbf{A} \\
\mathbf{A}\end{array}$ & $\begin{array}{l}6384.24 \\
6384.78 \\
6386.82 \\
6388.06 \\
6389.85\end{array}$ & $\begin{array}{l}1 \\
1 \\
3 \\
1 \\
3\end{array}$ & $\begin{array}{l}\mathbf{E} \\
\mathbf{E} \\
\mathbf{A} \\
\mathbf{A} \\
\mathbf{A}\end{array}$ & $\begin{array}{l}6498.31 \\
6498.67 \\
6500.95 \\
6502.00 \\
6504.70\end{array}$ & $\begin{array}{l}1 \\
3 \\
1 \\
2 \\
1\end{array}$ & $\begin{array}{l}\mathbf{B} \\
\mathbf{B} \\
\mathbf{E} \\
\mathbf{A} \\
\mathbf{E}\end{array}$ \\
\hline $\begin{array}{l}6275.03 \\
6275.74 \\
6276.73 \\
6278.20 \\
6279.53\end{array}$ & $\begin{array}{l}1 \\
1 \\
1 \\
1 \\
1\end{array}$ & $\begin{array}{l}\mathbf{A} \\
\mathbf{A} \\
\mathbf{B} \\
\mathbf{E} \\
\mathbf{B}\end{array}$ & $\begin{array}{l}6390.81 \\
6392.37 \\
6394.04 \\
6395.34 \\
6398.29\end{array}$ & $\begin{array}{l}3 \\
1 \\
1 \\
1 \\
1 \\
1\end{array}$ & $\begin{array}{l}\mathbf{A} \\
\mathbf{E} \\
\mathbf{E} \\
\mathbf{E} \\
\mathbf{A}\end{array}$ & $\begin{array}{l}6505.51 \\
6507.13 \\
6507.70 \\
6509.45 \\
6512.69\end{array}$ & $\begin{array}{l}1 \mathrm{~b} \\
1 \\
21 \\
3 \mathrm{~b}, 1 \\
2\end{array}$ & $\begin{array}{l}\mathbf{E} \\
\mathbf{B} \\
\mathbf{A} \\
\mathbf{C} \\
\mathbf{B}\end{array}$ \\
\hline $\begin{array}{l}6281.85 \\
6284.12 \\
6285.13 \\
6287.72 \\
6290.71\end{array}$ & $\begin{array}{l}1 \\
1 \\
1 \\
1 \\
1\end{array}$ & $\begin{array}{l}\mathbf{E} \\
\mathbf{A} \\
\mathbf{A} \\
\mathbf{B} \\
\mathbf{E}\end{array}$ & $\begin{array}{l}6399.39 \\
6399.86 \\
6401.45 \\
6402.10 \\
6403.99\end{array}$ & $\begin{array}{l}1 \\
1 \\
1 \\
1 \\
2 v\end{array}$ & $\begin{array}{l}\mathbf{E} \\
\mathbf{E} \\
\mathbf{E} \\
\mathbf{E} \\
\mathbf{A}\end{array}$ & $\begin{array}{l}6516.14 \\
6516.62 \\
6525.51 \\
6526.64 \\
6528.00\end{array}$ & $\begin{array}{l}1 \\
1 \\
1 \\
2 \\
2\end{array}$ & $\begin{array}{l}\text { B } \\
\text { B } \\
\mathbf{E} \\
\mathbf{A} \\
\mathbf{A}\end{array}$ \\
\hline $\begin{array}{l}6291.82 \\
6292.92 \\
6293.91 \\
6294.68 \\
6295.55\end{array}$ & $\begin{array}{l}3 \\
2 \\
1 \\
2 \\
1\end{array}$ & $\begin{array}{l}A \\
A \\
A \\
A \\
\text { E }\end{array}$ & $\begin{array}{l}6404.52 \\
6405.53 \\
6406.24 \\
6408.06 \\
6410.26\end{array}$ & $\begin{array}{l}1 \\
1 \\
2 \\
1 \\
1\end{array}$ & $\begin{array}{l}\mathbf{E} \\
\mathbf{A} \\
\mathbf{A} \\
\mathbf{E} \\
\mathbf{E}\end{array}$ & $\begin{array}{l}6528.91 \\
6529.64 \\
6530.68 \\
6532.21 \\
6533.12\end{array}$ & $\begin{array}{l}1 \\
2 \\
1 \\
1 \\
1\end{array}$ & $\begin{array}{l}\mathbf{E} \\
\mathrm{A} \\
\mathrm{E} \\
\mathrm{B} \\
\mathrm{B}\end{array}$ \\
\hline $\begin{array}{l}6295.99 \\
6297.64 \\
6300.19 \\
6301.12 \\
630183\end{array}$ & $\begin{array}{l}1 \\
1 \\
2 \\
2 \\
1\end{array}$ & $\begin{array}{l}\mathrm{A} \\
\mathrm{A} \\
\mathrm{A} \\
\mathrm{A} \\
\mathrm{A}\end{array}$ & $\begin{array}{l}6411.40 \\
6417.50 \\
6418.86 \\
6419.32 \\
6424.18\end{array}$ & $\begin{array}{l}2 \\
3 \\
1 \\
1 \\
1\end{array}$ & $\begin{array}{l}\text { B } \\
\text { A } \\
\text { E } \\
\text { E } \\
\text { E }\end{array}$ & $\begin{array}{l}6533.90 \\
6536.78 \\
6537.52 \\
6542.76 \\
6543.29\end{array}$ & $\begin{array}{l}2 \\
1 \\
1 \\
3 \\
1\end{array}$ & $\begin{array}{l}\text { E } \\
\text { E } \\
\text { E } \\
\text { A } \\
\text { B }\end{array}$ \\
\hline $\begin{array}{l}6302.39 \\
6303.15 \\
6306.25 \\
6309.87 \\
6310.24\end{array}$ & $\begin{array}{l}2 \\
2 \\
1 \\
1 \\
1\end{array}$ & $\begin{array}{l}\text { A } \\
\text { A } \\
\text { B } \\
\text { A } \\
\text { E }\end{array}$ & $\begin{array}{l}6425.90 \\
6426.61 \\
6428.32 \\
6428.92 \\
6431.00\end{array}$ & $\begin{array}{l}2 \\
4 \\
2 \\
2 \\
3\end{array}$ & $\begin{array}{l}\text { A } \\
\text { A } \\
\text { A } \\
\text { A } \\
\text { B }\end{array}$ & $\begin{array}{l}6544.57 \\
6544.91 \\
6549.77 \\
6550.97 \\
6551.78\end{array}$ & $\begin{array}{l}2 \\
1 \\
3 \\
1 \\
2 \\
C\end{array}$ & $\begin{array}{l}\mathbf{A} \\
\mathbf{E} \\
\mathbf{A} \\
\mathrm{A} \\
\mathrm{A}\end{array}$ \\
\hline $\begin{array}{l}6311.47 \\
6313.16 \\
6314.60 \\
6315.78 \\
6319.00\end{array}$ & $\begin{array}{l}1 \\
1 \\
1 \\
2 \\
1\end{array}$ ES & $\begin{array}{l}\mathbf{E} \\
\mathbf{E} \\
\mathbf{E} \\
\mathbf{A} \\
\mathbf{E}\end{array}$ & $\begin{array}{l}6431.96 \\
6433.92 \\
6435.29 \\
6437.58 \\
6438.00\end{array}$ & $\begin{array}{l}2 \\
2 \\
2 \\
2 \\
2\end{array}$ & $\begin{array}{l}\text { B } \\
\text { B } \\
\text { B } \\
\text { B } \\
\text { B }\end{array}$ & $\begin{array}{l}6556.40 \\
6557.00 \\
6558.26 \\
6562.94 \\
6563.48\end{array}$ & $\begin{array}{l}1 \\
1 \\
2 \\
2 \\
1\end{array}$ & $\begin{array}{l}\mathrm{E} \\
\mathbf{B} \\
\mathrm{A} \\
\mathrm{A} \\
\mathrm{C}\end{array}$ \\
\hline $\begin{array}{l}6321.75 \\
6322.48 \\
6325.54 \\
6326.23 \\
6327.47\end{array}$ & $\begin{array}{l}3 \\
1 \\
3 \\
1 \\
3\end{array}$ & $\begin{array}{l}\mathbf{A} \\
\mathbf{A} \\
\mathbf{A} \\
\mathbf{E} \\
\mathbf{A}\end{array}$ & $\begin{array}{l}6439.72 \\
6440.54 \\
6442.03 \\
6443.61 \\
6447.49\end{array}$ & $\begin{array}{l}2 \\
2 \\
1 \\
1 \\
2\end{array}$ & $\begin{array}{l}\text { B } \\
\text { B } \\
\mathbf{E} \\
\text { B } \\
\text { B }\end{array}$ & $\begin{array}{l}6564.60 \\
6566.41 \\
6569.31 \\
6570.67 \\
6572.98\end{array}$ & $\begin{array}{l}1 \\
2 \\
6 \\
4 \\
1\end{array}$ & $\begin{array}{l}\mathbf{E} \\
\mathbf{B} \\
\mathbf{A} \\
\mathbf{A} \\
\mathbf{A}\end{array}$ \\
\hline $\begin{array}{l}6328.00 \\
6328.84 \\
6329.35 \\
6331.17 \\
6331.75\end{array}$ & $\begin{array}{l}1 \\
1 \\
1 \\
1 \\
1 \\
1\end{array}$ & $\begin{array}{l}\mathbf{A} \\
\mathbf{A} \\
\mathbf{A} \\
\mathbf{E} \\
\mathbf{C}\end{array}$ & $\begin{array}{l}6448.03 \\
6448.25 \\
6450.41 \\
6452.06 \\
6455.60\end{array}$ & $\begin{array}{l}1 \\
1 \\
1 \\
2 \\
2 \mathrm{Ca} ?\end{array}$ & $\begin{array}{l}\text { B } \\
\text { B } \\
\text { B } \\
\text { B } \\
\text { B }\end{array}$ & $\begin{array}{l}6574.38 \\
6575.95 \\
6577.36 \\
6579.21 \\
6580.46\end{array}$ & $\begin{array}{l}3 \\
2 \\
1 \\
1 \\
1\end{array}$ & $\begin{array}{l}\mathbf{A} \\
\mathbf{A} \\
\mathbf{B} \\
\mathbf{E} \\
\mathbf{A}\end{array}$ \\
\hline $\begin{array}{l}6335.37 \\
6337.87 \\
6338.90 \\
6340.07 \\
6341.41\end{array}$ & $\begin{array}{l}1 \\
1 \\
1 \\
2 \\
1 \\
1\end{array}$ & $\begin{array}{l}\mathrm{B} \\
\mathrm{B} \\
\mathrm{A} \\
\mathrm{A} \\
\mathrm{E}\end{array}$ & $\begin{array}{l}6456.25 \\
6457.53 \\
6459.33 \\
6468.28 \\
6468.91\end{array}$ & $\begin{array}{l}2 \\
1 \\
1 \\
1 \\
1 \\
1\end{array}$ & $\begin{array}{l}\mathbf{B} \\
\mathbf{B} \\
\mathbf{B} \\
\mathbf{E} \\
\mathbf{E}\end{array}$ & $\begin{array}{l}6581.15 \\
6584.06 \\
6585.21 \\
6587.50 \\
6588.32\end{array}$ & $\begin{array}{l}1 \\
1 \\
4 \\
2 \\
1\end{array}$ & $\begin{array}{l}\mathbf{B} \\
\mathbf{B} \\
\mathbf{A} \\
\mathbf{A} \\
\mathbf{A}\end{array}$ \\
\hline $\begin{array}{l}6342.14 \\
6343.32 \\
6344.01 \\
6348.92 \\
6349.20\end{array}$ & $\begin{array}{l}1 \\
1 \\
1 \\
1 \\
1\end{array}$ & $\begin{array}{l}\mathbf{A} \\
\mathbf{E} \\
\mathbf{E} \\
\mathbf{A} \\
\mathbf{B}\end{array}$ & $\begin{array}{l}6469.69 \\
6470.46 \\
6471.57 \\
6472.34 \\
6473.24\end{array}$ & $\begin{array}{l}1 \mathrm{v} \\
3 \\
3 \\
4 \\
1\end{array}$ & $\begin{array}{l}\mathbf{E} \\
\mathbf{B} \\
\mathbf{B} \\
\mathrm{B} \\
\mathrm{E}\end{array}$ & $\begin{array}{l}6588.91 \\
6589.72 \\
6591.53 \\
6592.96 \\
6595.96\end{array}$ & $\begin{array}{l}3 \\
5 \\
2 \\
1 \\
1\end{array}$ & $\begin{array}{l}\text { A } \\
\text { A } \\
\text { A } \\
\text { E } \\
\text { A }\end{array}$ \\
\hline
\end{tabular}


TABLE 2.-Arc Spectrum of Samarium-Continued

\begin{tabular}{|c|c|c|c|c|c|c|c|c|}
\hline XI. A. & Notes & $\begin{array}{c}\text { Probabie } \\
\text { error }\end{array}$ & $\lambda I . A$. & Notes & $\begin{array}{l}\text { Probable } \\
\text { error }\end{array}$ & 入 1. A. & Notes & $\begin{array}{l}\text { Probable } \\
\text { error }\end{array}$ \\
\hline $\begin{array}{l}6596.96 \\
6597.65 \\
6598.88 \\
6601.83 \\
6603.50\end{array}$ & $\begin{array}{l}2 \\
1 \\
1 \\
5 \\
1\end{array}$ & $\begin{array}{l}\mathbf{A} \\
\mathbf{B} \\
\mathbf{E} \\
\mathbf{A} \\
\mathbf{B}\end{array}$ & $\begin{array}{l}6718.22 \\
6719.59 \\
6720.91 \\
6721.56 \\
6723.15\end{array}$ & $\begin{array}{l}1 \\
1 \\
1 \\
1 \\
4 \\
4\end{array}$ & $\begin{array}{l}\text { E } \\
\text { B } \\
\text { A } \\
\text { A } \\
\text { A }\end{array}$ & $\begin{array}{l}6848.87 \\
6853.50 \\
6853.88 \\
6854.49 \\
6856.03\end{array}$ & $\begin{array}{l}1 \\
1 \\
1 \\
2 \\
5\end{array}$ & $\begin{array}{l}\text { B } \\
\text { A } \\
\text { A } \\
\text { A } \\
\text { A }\end{array}$ \\
\hline $\begin{array}{l}6604.56 \\
6607.08 \\
6612.49 \\
6614.82 \\
6616.74\end{array}$ & $\begin{array}{l}5 \\
1 \\
1 \\
2 \\
1\end{array}$ & $\begin{array}{l}\mathbf{A} \\
\mathbf{E} \\
\mathbf{E} \\
\mathbf{A} \\
\mathbf{B}\end{array}$ & $\begin{array}{l}6724.73 \\
6725.89 \\
6726.81 \\
6728.18 \\
6731.84\end{array}$ & $\begin{array}{l}3 \\
3 \\
1 \\
1 \\
\frac{1}{6}, \mathrm{I}\end{array}$ & $\begin{array}{l}\mathrm{A} \\
\mathrm{A} \\
\mathrm{B} \\
\mathrm{A} \\
\mathrm{A}\end{array}$ & $\begin{array}{l}6858.12 \\
6861.12 \\
6862.82 \\
6867.08 \\
6872.43\end{array}$ & $\begin{array}{l}1 \\
6 \\
4 \\
1 \\
3 \\
3\end{array}$ & $\begin{array}{l}\text { A } \\
\text { A } \\
\text { A } \\
\text { B } \\
\text { A }\end{array}$ \\
\hline $\begin{array}{l}6617.61 \\
6619.01 \\
6625.28 \\
6626.88 \\
6627.45\end{array}$ & $\begin{array}{l}2 \\
1 \\
2 \\
1 \\
1\end{array}$ & $\begin{array}{l}\mathbf{A} \\
\mathbf{E} \\
\mathbf{B} \\
\mathbf{B} \\
\mathbf{E}\end{array}$ & $\begin{array}{l}6734.05 \\
6734.81 \\
6735.34 \\
6746.41 \\
6750.82\end{array}$ & $\begin{array}{l}5 \\
4 \\
2 \\
1 \mathrm{~b} \\
1 \mathrm{~b}\end{array}$ & $\begin{array}{l}\mathrm{A} \\
\mathrm{A} \\
\mathrm{A} \\
\mathrm{B} \\
\mathrm{B}\end{array}$ & $\begin{array}{l}6873.60 \\
6875.25 \\
6877.10 \\
6878.34 \\
6879.51\end{array}$ & $\begin{array}{l}1 \\
2 \\
2 \\
1 \\
2\end{array}$ & $\begin{array}{l}\mathrm{E} \\
\mathbf{B} \\
\mathbf{A} \\
\mathrm{E} \\
\mathrm{A}\end{array}$ \\
\hline $\begin{array}{l}6627.76 \\
6628.88 \\
6630.61 \\
6632.28 \\
6633.05\end{array}$ & $\begin{array}{l}1 \\
2 \\
2 \\
4 \\
1\end{array}$ & $\begin{array}{l}\mathbf{E} \\
\mathbf{A} \\
\mathbf{A} \\
\mathbf{A} \\
\mathbf{E}\end{array}$ & $\begin{array}{l}6754.80 \\
6756.94 \\
6759.24 \\
6761.64 \\
6762.06\end{array}$ & $\begin{array}{l}2 \\
1 \\
2 \\
1 \\
1\end{array}$ & $\begin{array}{l}\mathrm{A} \\
\mathrm{A} \\
\mathrm{A} \\
\mathrm{E} \\
\mathrm{B}\end{array}$ & $\begin{array}{l}6880.86 \\
6883.39 \\
6885.16 \\
6887.42 \\
6888.79\end{array}$ & $\begin{array}{l}1 \\
1 \\
3 \\
1 \\
2\end{array}$ & $\begin{array}{l}\text { B } \\
\text { E } \\
\text { A } \\
\text { B } \\
\text { A }\end{array}$ \\
\hline $\begin{array}{l}6635.18 \\
6638.49 \\
6639.32 \\
6639.75 \\
6640.60\end{array}$ & $\begin{array}{l}2 \\
1 \\
1 \\
1 \\
1 \mathrm{~b}, \mathrm{~d} \text { ? }\end{array}$ & $\begin{array}{l}\mathbf{B} \\
\mathbf{B} \\
\mathbf{B} \\
\mathbf{B} \\
\mathbf{C}\end{array}$ & $\begin{array}{l}6762.58 \\
6764.23 \\
6776.52 \\
6769.40 \\
6770.37\end{array}$ & $\begin{array}{l}1 \\
1 \\
21 \\
1 \mathrm{v} \\
1\end{array}$ & $\begin{array}{l}\mathbf{E} \\
\mathbf{B} \\
\mathbf{A} \\
\mathbf{B} \\
\mathbf{B}\end{array}$ & $\begin{array}{l}6891.46 \\
6892.00 \\
6900.28 \\
6904.51 \\
6906.18\end{array}$ & $\begin{array}{l}2 \\
1 \\
2 \\
1 \\
1\end{array}$ & $\begin{array}{l}\mathbf{A} \\
\mathbf{A} \\
\mathbf{B} \\
\mathbf{A} \\
\mathbf{E}\end{array}$ \\
\hline $\begin{array}{l}6641.49 \\
6643.79 \\
6645.08 \\
6646.18 \\
6648.23\end{array}$ & $\begin{array}{l}1 v \\
1 \\
1 \\
2 \\
2\end{array}$ & $\begin{array}{l}\mathrm{E} \\
\mathbf{C} \\
\mathrm{E} \\
\mathbf{A} \\
\mathbf{B}\end{array}$ & $\begin{array}{l}6771.31 \\
6771.98 \\
6775.35 \\
6778.19 \\
6778.61\end{array}$ & $\begin{array}{l}1 \\
1 \\
2 \\
3 \\
4 \\
4\end{array}$ & $\begin{array}{l}\text { A } \\
\text { A } \\
\text { B } \\
\text { A } \\
\text { A }\end{array}$ & $\begin{array}{l}6909.81 \\
6918.96 \\
6925.19 \\
6926.15 \\
6927.02\end{array}$ & $\begin{array}{l}2 \\
2 \\
1 \\
1 \\
2\end{array}$ & $\begin{array}{l}\text { A } \\
\text { A } \\
\text { B } \\
\text { B } \\
\text { A }\end{array}$ \\
\hline $\begin{array}{l}6649.02 \\
6651.61 \\
6654.24 \\
6656.19 \\
6661.32\end{array}$ & $\begin{array}{l}3 \\
3 \\
1 \\
3 \\
1\end{array}$ & $\begin{array}{l}\text { A } \\
\text { A } \\
\text { B } \\
\text { A } \\
\text { B }\end{array}$ & $\begin{array}{l}6779.15 \\
6780.03 \\
6781.17 \\
6782.95 \\
6784.35\end{array}$ & $\begin{array}{l}1 \\
2 \\
2 \\
3 \\
1 v\end{array}$ & $\begin{array}{l}\mathrm{A} \\
\mathrm{A} \\
\mathrm{A} \\
\mathrm{A} \\
\mathrm{C}\end{array}$ & $\begin{array}{l}6928.82 \\
6929.59 \\
6930.41 \\
6934.28 \\
6937.55\end{array}$ & $\begin{array}{l}1 \mathrm{~b} \\
1 \\
2 \\
1 \\
1\end{array}$ & $\begin{array}{l}\text { B } \\
\text { B } \\
\text { A } \\
\text { B } \\
\text { B }\end{array}$ \\
\hline $\begin{array}{l}6661.58 \\
6667.22 \\
6669.31 \\
6671.49 \\
6672.13\end{array}$ & $\begin{array}{l}1 \\
3 \\
1 \\
4 \\
1\end{array}$ & $\begin{array}{l}\text { B } \\
\text { A } \\
\text { E } \\
\text { A } \\
\text { E }\end{array}$ & $\begin{array}{l}6790.00 \\
6790.83 \\
6791.43 \\
6792.55 \\
6794.20\end{array}$ & $\begin{array}{l}5 \\
1 \\
1 \\
2 \mathrm{~b} \\
5\end{array}$ & $\begin{array}{l}\mathbf{A} \\
\mathbf{A} \\
\mathbf{E} \\
\mathbf{A} \\
\mathbf{A}\end{array}$ & $\begin{array}{l}6938.74 \\
6939.51 \\
6941.56 \\
6948.25 \\
6949.22\end{array}$ & $\begin{array}{l}1 \\
1 \\
2 \\
1 \\
2 \mathrm{~b}\end{array}$ & $\begin{array}{l}\mathrm{B} \\
\mathrm{B} \\
\mathrm{A} \\
\mathrm{E} \\
\mathrm{B}\end{array}$ \\
\hline $\begin{array}{l}6674.68 \\
6675.55 \\
6676.99 \\
6677.46 \\
6679.21\end{array}$ & $\begin{array}{l}1 \\
1 \\
2 \\
1 \\
5\end{array}$ & $\begin{array}{l}\mathrm{E} \\
\mathbf{B} \\
\mathbf{A} \\
\mathbf{E} \\
\mathbf{A}\end{array}$ & $\begin{array}{l}6796.13 \\
6796.82 \\
6797.59 \\
6801.79 \\
6804.86\end{array}$ & $\begin{array}{l}1 \\
2 \\
1 \\
1 \\
1\end{array}$ & $\begin{array}{l}\mathbf{A} \\
\mathbf{A} \\
\mathbf{E} \\
\mathbf{E} \\
\mathbf{E}\end{array}$ & $\begin{array}{l}6950.51 \\
6952.42 \\
6953.61 \\
6954.32 \\
6955.29\end{array}$ & $\begin{array}{l}3 \\
1 \\
1 \\
1 \\
5\end{array}$ & $\begin{array}{l}\text { A } \\
\text { E } \\
\text { B } \\
\text { B } \\
\text { A }\end{array}$ \\
\hline $\begin{array}{l}6681.53 \\
6682.53 \\
6684.42 \\
6685.02 \\
6686.30\end{array}$ & $\begin{array}{l}3 \\
1 \\
1 \\
1 \\
1\end{array}$ & $\begin{array}{l}\mathrm{A} \\
\mathrm{B} \\
\mathrm{B} \\
\mathrm{E} \\
\mathrm{B}\end{array}$ & $\begin{array}{l}6805.58 \\
6807.50 \\
6808.30 \\
6813.42 \\
6814.87\end{array}$ & $\begin{array}{l}1 \\
1 \\
2 \\
1 \mathrm{~b} \\
1\end{array}$ & $\begin{array}{l}\mathbf{E} \\
\mathbf{B} \\
\mathbf{A} \\
\mathbf{B} \\
\mathbf{E}\end{array}$ & $\begin{array}{l}6958.98 \\
6965.95 \\
6966.49 \\
6968.65 \\
6969.69\end{array}$ & $\begin{array}{l}1 \\
1 \\
1 \\
2 b, 1 \\
1\end{array}$ & $\begin{array}{l}\mathbf{B} \\
\mathbf{B} \\
\mathbf{B} \\
\mathbf{B} \\
\mathbf{B}\end{array}$ \\
\hline $\begin{array}{l}6687.79 \\
6688.78 \\
6693.55 \\
6694.69 \\
6696.77\end{array}$ & $\begin{array}{l}2 \\
1 \\
5 \\
2 \\
1\end{array}$ & $\begin{array}{l}\mathbf{A} \\
\mathbf{B} \\
\mathbf{A} \\
\mathbf{A} \\
\mathbf{A}\end{array}$ & $\begin{array}{l}6815.50 \\
6815.14 \\
6820.91 \\
6821.87 \\
6823.61\end{array}$ & $\begin{array}{l}1 \\
\frac{1}{2} \\
1 \\
1\end{array}$ & $\begin{array}{l}\text { B } \\
\text { B } \\
\text { A } \\
\text { A } \\
\text { B }\end{array}$ & $\begin{array}{l}6975.58 \\
6975.99 \\
6977.58 \\
6984.16 \\
6985.67\end{array}$ & $\begin{array}{l}1 \\
1 \\
1 \\
1 \\
1\end{array}$ & $\begin{array}{l}\text { E } \\
\text { B } \\
\text { B } \\
\text { B } \\
\text { B }\end{array}$ \\
\hline $\begin{array}{l}6697.23 \\
6698.05 \\
6698.92 \\
6702.19 \\
6703.60\end{array}$ & $\begin{array}{l}1 \\
1 \\
1 \\
1 \\
2\end{array}$ & $\begin{array}{l}\mathbf{A} \\
\mathbf{E} \\
\mathbf{E} \\
\mathbf{A} \\
\mathbf{A}\end{array}$ & $\begin{array}{l}6826.68 \\
6827.05 \\
6827.74 \\
6828.47 \\
6829.17\end{array}$ & $\begin{array}{l}1 \\
1 \\
1 \\
1 \\
1\end{array}$ & $\begin{array}{l}\mathbf{E} \\
\mathbf{E} \\
\mathbf{A} \\
\mathbf{A} \\
\mathbf{B}\end{array}$ & $\begin{array}{l}6987.34 \\
6988.34 \\
6990.08 \\
6993.41 \\
7007.39\end{array}$ & $\begin{array}{l}1 \\
2 \\
1 \\
1 \\
1\end{array}$ & $\begin{array}{l}\mathbf{B} \\
\mathbf{A} \\
\mathbf{B} \\
\mathbf{B} \\
\mathbf{B}\end{array}$ \\
\hline $\begin{array}{l}6704.26 \\
6705.48 \\
6706.78 \\
6707.47 \\
6710.70\end{array}$ & $\begin{array}{l}1 \\
1 \\
1 \\
3 \\
1\end{array}$ & $\begin{array}{l}\text { A } \\
\text { B } \\
\text { E } \\
\text { A } \\
\text { B }\end{array}$ & $\begin{array}{l}6829.85 \\
6830.52 \\
6837.15 \\
6838.28 \\
6839.08\end{array}$ & $\begin{array}{l}3 \\
2 \\
1 \\
2 \\
1\end{array}$ & $\begin{array}{l}\text { A } \\
\text { A } \\
\text { B } \\
\text { A } \\
\text { B }\end{array}$ & $\begin{array}{l}7008.45 \\
7009.79 \\
7015.37 \\
7016.76 \\
7020.44\end{array}$ & $\begin{array}{l}1 \\
1 \\
1 \\
\frac{1}{5}\end{array}$ & $\begin{array}{l}\text { B } \\
\text { B } \\
\mathbf{E} \\
\mathbf{C} \\
\mathbf{A}\end{array}$ \\
\hline $\begin{array}{l}6711.46 \\
6712.13 \\
6712.62 \\
6713.79 \\
6717.08\end{array}$ & $\begin{array}{l}1 \\
1 \\
3 \\
1 \\
1\end{array}$ & $\begin{array}{l}\mathrm{A} \\
\mathrm{A} \\
\mathrm{A} \\
\mathrm{B} \\
\mathrm{B}\end{array}$ & $\begin{array}{l}6839.65 \\
6841.76 \\
6844.70 \\
6846.54 \\
6848.15\end{array}$ & $\begin{array}{l}1 \\
2 \\
4 \\
3 \\
3 \mathrm{~b}\end{array}$ & $\begin{array}{l}\mathbf{B} \\
\mathbf{A} \\
\mathbf{A} \\
\mathbf{A} \\
\mathbf{A}\end{array}$ & $\begin{array}{l}7021.48 \\
7022.66 \\
7023.74 \\
7026.62 \\
7036.72\end{array}$ & $\begin{array}{l}1 \\
1 \\
1 \mathrm{v} \\
2 \mathrm{2} \\
2 \mathrm{~b}\end{array}$ & $\begin{array}{l}\mathbf{E} \\
\mathbf{B} \\
\mathbf{B} \\
\mathbf{B} \\
\mathbf{B}\end{array}$ \\
\hline
\end{tabular}


TABLE 2.-Arc Spectrum of Samarium-Continued

\begin{tabular}{|c|c|c|c|c|c|c|c|c|}
\hline$\lambda$ I. A. & Notes & $\begin{array}{c}\text { Probable } \\
\text { error }\end{array}$ & $\lambda$ I. A. & Notes & $\begin{array}{c}\text { Probable } \\
\text { error }\end{array}$ & $\lambda$ I. A. & Notes & $\begin{array}{c}\text { Probable } \\
\text { ertor }\end{array}$ \\
\hline $\begin{array}{l}7039.22 \\
7042.24 \\
7049.15 \\
7051.52 \\
7054.97\end{array}$ & $\begin{array}{l}6 \\
5 \\
1 \\
5 \\
1\end{array}$ & $\begin{array}{l}\text { B } \\
\text { A } \\
\text { B } \\
\text { B } \\
\text { B }\end{array}$ & $\begin{array}{l}7279.25 \\
7281.50 \\
7282.28 \\
7283.38 \\
7288.93\end{array}$ & $\begin{array}{l}1 \\
1 \\
1 \\
1 \\
1\end{array}$ & $\begin{array}{l}\text { B } \\
\text { B } \\
\text { B } \\
\text { B } \\
\text { B }\end{array}$ & $\begin{array}{l}7863.64 \\
7873.71 \\
7875.11 \\
7880.06 \\
7895.96\end{array}$ & $\begin{array}{l}1 \\
1 \\
1 \\
2 \\
1\end{array}$. & $\begin{array}{l}\mathrm{A} \\
\mathbf{E} \\
\mathbf{E} \\
\mathrm{A} \\
\mathrm{C}\end{array}$ \\
\hline $\begin{array}{l}7056.53 \\
7064.97 \\
7065.84 \\
7067.36 \\
7071.10\end{array}$ & $\begin{array}{l}1 \\
1 \text { b } \\
1 \text { b } \\
1 \\
1\end{array}$ & $\begin{array}{l}\text { B } \\
\text { B } \\
\text { B } \\
\text { B } \\
\text { B }\end{array}$ & $\begin{array}{l}7290.23 \\
7300.72 \\
7316.34 \\
7327.09 \\
7332.65\end{array}$ & $\begin{array}{l}1 \\
1 \text { b } \\
1 \\
1 \\
1\end{array}$ & $\begin{array}{l}\text { B } \\
\text { B } \\
\text { E } \\
\text { A } \\
\text { A }\end{array}$ & $\begin{array}{l}7906.26 \\
7914.96 \\
7928.19 \\
7937.04 \\
7940.31\end{array}$ & $\begin{array}{l}1 \\
2 \\
2 \\
1 \\
1\end{array}$ & $\begin{array}{l}\mathrm{E} \\
\mathrm{B} \\
\mathrm{B} \\
\mathrm{E} \\
\mathrm{E}\end{array}$ \\
\hline $\begin{array}{l}7074.67 \\
7079.50 \\
7082.37 \\
7085.52 \\
7088.30\end{array}$ & $\begin{array}{l}1 \\
1 \\
5 \\
2 \mathrm{~b}, \mathrm{~d} ?\end{array}$ & $\begin{array}{l}\text { B } \\
\text { E } \\
\text { B } \\
\text { B } \\
\text { B }\end{array}$ & $\begin{array}{l}7335.08 \\
7338.04 \\
7344.84 \\
7347.30 \\
7362.99\end{array}$ & $\begin{array}{l}1 \mathbf{b} \\
1 \\
1 \\
2 \\
1\end{array}$ & $\begin{array}{l}\mathbf{E} \\
\mathbf{A} \\
\mathbf{E} \\
\mathbf{A} \\
\mathbf{E}\end{array}$ & $\begin{array}{l}7948.12 \\
7968.34 \\
8001.59 \\
8004.97 \\
8014.89\end{array}$ & $\begin{array}{l}1 \\
1 \\
2 \\
1 \\
2\end{array}$ & $\begin{array}{l}\mathrm{E} \\
\mathrm{E} \\
\mathrm{A} \\
\mathrm{B} \\
\mathrm{B}\end{array}$ \\
\hline $\begin{array}{l}7091.16 \\
7092.98 \\
7096.33 \\
7100.75 \\
7102.02\end{array}$ & $\begin{array}{l}2 \\
1 \text { b } \\
2 \\
1 \\
1 b\end{array}$ & $\begin{array}{l}\mathrm{B} \\
\mathrm{B} \\
\mathrm{B} \\
\mathrm{E} \\
\mathrm{B}\end{array}$ & $\begin{array}{l}7364.22 \\
7371.50 \\
7373.73 \\
7375.22 \\
7376.69\end{array}$ & $\begin{array}{l}1 \\
1 \\
1 \\
1 \\
1\end{array}$ & $\begin{array}{l}\mathbf{E} \\
\mathrm{A} \\
\mathbf{E} \\
\mathbf{E} \\
\mathbf{A}\end{array}$ & $\begin{array}{l}8017.18 \\
8019.56 \\
8021.61 \\
8025.12 \\
8029.56\end{array}$ & $\begin{array}{l}1 \\
1 \\
1 \\
3 \\
1\end{array}$ & $\begin{array}{l}\mathbf{E} \\
\mathrm{C} \\
\mathrm{B} \\
\mathrm{A} \\
\mathrm{C}\end{array}$ \\
\hline $\begin{array}{l}7104.53 \\
7106.21 \\
7106.73 \\
7110.57 \\
7111.43\end{array}$ & $\begin{array}{l}2 \\
2 \\
1 \\
1 \\
1\end{array}$ & $\begin{array}{l}\text { B } \\
\text { B } \\
\text { B } \\
\text { E } \\
\text { E }\end{array}$ & $\begin{array}{l}7383.54 \\
7393.35 \\
7394.05 \\
704.00 \\
7412.15\end{array}$ & $\begin{array}{l}1 \\
1 \\
1 \\
1 \\
1\end{array}$ & $\begin{array}{l}\text { A } \\
\text { B } \\
\text { B } \\
\text { A } \\
\text { E }\end{array}$ & $\begin{array}{l}8032.02 \\
8033.70 \\
8038.38 \\
8040.27 \\
8048.70\end{array}$ & $\begin{array}{l}2 \\
1 \\
1 \\
1 \\
3\end{array}$ & $\begin{array}{l}\text { A } \\
\text { C } \\
\text { D } \\
\text { C } \\
\text { A }\end{array}$ \\
\hline $\begin{array}{l}7112.72 \\
7114.53 \\
7115.28 \\
7115.97 \\
7117.52\end{array}$ & $\begin{array}{l}1 \\
1 \\
1 \\
2 \\
1\end{array}$ & $\begin{array}{l}\mathrm{E} \\
\mathrm{E} \\
\mathrm{E} \\
\mathrm{B} \\
\mathrm{B}\end{array}$ & $\begin{array}{l}7444.56 \\
7445.41 \\
7470.72 \\
7481.99 \\
7494.02\end{array}$ & $\begin{array}{l}2 \\
2 \\
1 \\
1 \\
1\end{array}$ & $\begin{array}{l}\mathrm{A} \\
\mathrm{A} \\
\mathrm{E} \\
\mathrm{B} \\
\mathrm{E}\end{array}$ & $\begin{array}{l}8065.16 \\
8068.47 \\
8007.37 \\
8071.74 \\
8076.29\end{array}$ & $\begin{array}{l}1 \\
4 \\
1 \\
2 \\
1\end{array}$ & $\begin{array}{l}\mathrm{B} \\
\mathrm{A} \\
\mathrm{B} \\
\mathrm{B} \\
\mathrm{B}\end{array}$ \\
\hline $\begin{array}{l}7118.28 \\
7119.81 \\
7122.41 \\
7123.29 \\
7125.11\end{array}$ & $\begin{array}{l}1 \\
1 \\
1 \\
1 \\
1\end{array}$ & $\begin{array}{l}\mathrm{E} \\
\mathrm{B} \\
\mathrm{B} \\
\mathrm{E} \\
\mathrm{B}\end{array}$ & $\begin{array}{l}7502.36 \\
7541.39 \\
7543.62 \\
7544.80 \\
7546.64\end{array}$ & $\begin{array}{l}1 \mathrm{~b} \\
11 \\
1 \\
1 \\
1\end{array}$ & $\begin{array}{l}\mathrm{B} \\
\mathrm{B} \\
\mathrm{E} \\
\mathrm{E} \\
\mathrm{E}\end{array}$ & $\begin{array}{l}8084.54 \\
8087.09 \\
8102.38 \\
8104.58 \\
8112.31\end{array}$ & $\begin{array}{l}1 \\
1 \\
1 \\
1 \\
1 \\
1\end{array}$ & $\begin{array}{l}\mathrm{B} \\
\mathrm{E} \\
\mathrm{B} \\
\mathrm{C} \\
\mathrm{B}\end{array}$ \\
\hline $\begin{array}{l}7131.80 \\
7135.99 \\
7136.43 \\
7137.56 \\
7138.01\end{array}$ & $\begin{array}{l}1 \\
1 \\
1 \\
1 \\
1\end{array}$ & $\begin{array}{l}\mathrm{B} \\
\mathrm{B} \\
\mathrm{E} \\
\mathrm{E} \\
\mathrm{E} \\
\mathrm{E}\end{array}$ & $\begin{array}{l}7560.23 \\
7562.90 \\
752.32 \\
7578.09 \\
7585.81\end{array}$ & $\begin{array}{l}1 \\
1 \\
1 \\
1 \\
1\end{array}$ & $\begin{array}{l}\mathbf{E} \\
\mathrm{B} \\
\mathbf{E} \\
\mathbf{A} \\
\mathbf{B}\end{array}$ & $\begin{array}{l}8116.20 \\
8117.17 \\
8121.22 \\
8122.36 \\
8125.12\end{array}$ & $\begin{array}{l}1 \\
2 \\
1 \\
1 \\
2 \\
2\end{array}$ & $\begin{array}{l}\mathrm{C} \\
\mathrm{A} \\
\mathrm{B} \\
\mathrm{E} \\
\mathrm{C}\end{array}$ \\
\hline $\begin{array}{l}7139.39 \\
7141.15 \\
7143.98 \\
7149.60 \\
7154.83\end{array}$ & $\begin{array}{l}1 \\
1 \\
2 \mathrm{~b} \\
3 \mathrm{~b} \\
1\end{array}$ & $\begin{array}{l}\mathbf{E} \\
\mathbf{E} \\
\mathbf{B} \\
\mathbf{B} \\
\mathbf{E}\end{array}$ & $\begin{array}{l}7588.40 \\
7607.59 \\
7638.09 \\
7645.08 \\
7645.82\end{array}$ & $\begin{array}{l}1 \\
1 \\
1 \\
1 \\
1 \\
1\end{array}$ & $\begin{array}{l}\text { E } \\
\text { B } \\
\text { E } \\
B \\
B\end{array}$ & $\begin{array}{l}8129.10 \\
8132.00 \\
8133.68 \\
8135.37 \\
8146.60\end{array}$ & $\begin{array}{l}2 \\
1 \\
1 \\
1 \\
1 \\
1\end{array}$ & $\begin{array}{l}\text { B } \\
\text { B } \\
\text { C } \\
\text { E } \\
\text { E }\end{array}$ \\
\hline $\begin{array}{l}7156.19 \\
7172.67 \\
7186.31 \\
7189.55 \\
7204.09\end{array}$ & $\begin{array}{l}1 \\
1 \\
1 \\
1 \\
1\end{array}$ & $\begin{array}{l}\mathbf{E} \\
\mathbf{E} \\
\mathbf{E} \\
\mathbf{E} \\
\mathbf{E}\end{array}$ & $\begin{array}{l}7648.03 \\
7667.19 \\
7695.78 \\
7728.56 \\
7736.26\end{array}$ & $\begin{array}{l}1 \\
1 \\
1 \\
1 \\
1\end{array}$ & $\begin{array}{l}\mathrm{B} \\
\mathrm{E} \\
\mathrm{E} \\
\mathrm{E} \\
\mathrm{E}\end{array}$ & $\begin{array}{l}8148.16 \\
8153.85 \\
8158.89 \\
8159.93 \\
8161.88\end{array}$ & $\begin{array}{l}1 \\
1 \\
1 \\
1 \\
3\end{array}$ & $\begin{array}{l}\text { E } \\
\text { B } \\
\text { E } \\
\text { B } \\
\text { A }\end{array}$ \\
\hline $\begin{array}{l}7210.95 \\
7213.82 \\
7218.09 \\
7220.10 \\
7225.62\end{array}$ & $\begin{array}{l}1 \\
1 \\
1 \mathbf{h} \\
1 \\
1\end{array}$ & $\begin{array}{l}\mathbf{E} \\
\mathbf{B} \\
\mathbf{E} \\
\mathbf{E} \\
\mathbf{B}\end{array}$ & $\begin{array}{l}7749.25 \\
7789.65 \\
7801.54 \\
7805.82 \\
7808.90\end{array}$ & $\begin{array}{l}1 \\
1 \\
1 \\
1 \\
1\end{array}$ & $\begin{array}{l}\mathbf{E} \\
\mathbf{E} \\
\mathbf{B} \\
\mathbf{E} \\
\mathbf{E}\end{array}$ & $\begin{array}{l}8165.38 \\
8167.25 \\
8172.96 \\
8180.34 \\
8184.42\end{array}$ & $\begin{array}{l}2 \\
1 \\
1 \\
1 \\
2\end{array}$ & $\begin{array}{l}\text { B } \\
\text { B } \\
\text { B } \\
\text { E } \\
\text { B }\end{array}$ \\
\hline $\begin{array}{l}7228.91 \\
7234.76 \\
7235.64 \\
7237.03 \\
7240.90\end{array}$ & $\begin{array}{l}1 \\
1 \\
1 \\
1 \\
2\end{array}$ & $\begin{array}{l}\mathbf{E} \\
\mathbf{E} \\
\mathbf{C} \\
\mathbf{E} \\
\mathbf{B}\end{array}$ & $\begin{array}{l}7812.71 \\
7813.60 \\
7820.12 \\
7831.39 \\
7835.08\end{array}$ & $\begin{array}{l}1 \\
1 \\
1 \\
1 \\
2\end{array}$ & $\begin{array}{l}\mathbf{B} \\
\mathbf{E} \\
\mathbf{B} \\
\mathbf{C} \\
\mathbf{A}\end{array}$ & $\begin{array}{l}8186.31 \\
8195.48 \\
8203.98 \\
8206.28 \\
8208.33\end{array}$ & $\begin{array}{l}2 \\
3 \\
2 \\
3 \\
3 \\
2\end{array}$ & $\begin{array}{l}\text { B } \\
\text { A } \\
\text { B } \\
\text { E } \\
\text { C }\end{array}$ \\
\hline $\begin{array}{l}7254.95 \\
7257.11 \\
7271.68 \\
7273.88 \\
7277.10\end{array}$ & $\begin{array}{l}1 \\
1 \\
1 \\
1\end{array}$ & $\begin{array}{l}\text { B } \\
\text { B } \\
\text { E } \\
\text { B } \\
\text { B }\end{array}$ & $\begin{array}{l}7837.27 \\
7844.79 \\
7846.10 \\
7848.73 \\
7859.53\end{array}$ & $\begin{array}{l}2 \\
1 \\
1 \\
1\end{array}$ & $\begin{array}{l}\mathrm{A} \\
\mathrm{B} \\
\mathrm{E} \\
\mathrm{C} \\
\mathrm{C}\end{array}$ & $\begin{array}{l}8214.74 \\
8218.76 \\
8230.34 \\
8240.98 \\
8244.97\end{array}$ & $\begin{array}{l}1 \\
3 \\
3 \\
3 \\
1\end{array}$ & $\begin{array}{l}\mathrm{D} \\
\mathrm{B} \\
\mathrm{B} \\
\mathrm{A} \\
\mathrm{B}\end{array}$ \\
\hline
\end{tabular}


TABLE 2.-Arc Spectrum of Samarium-Continued

\begin{tabular}{|c|c|c|c|c|c|c|c|c|}
\hline$\lambda$ I. A. & Notes & $\begin{array}{c}\text { Probable } \\
\text { error }\end{array}$ & $\lambda$ I. A. & Notes & $\begin{array}{c}\text { Probable } \\
\text { error }\end{array}$ & $\lambda$ I. A. & Notes & $\begin{array}{c}\text { Probable } \\
\text { error }\end{array}$ \\
\hline $\begin{array}{l}8246.52 \\
8248.15 \\
8249.38 \\
8252.53 \\
8258.06\end{array}$ & $\begin{array}{l}1 \\
1 \\
1 \\
1 \\
1\end{array}$ & $\begin{array}{l}\mathbf{B} \\
\mathbf{B} \\
\mathbf{B} \\
\mathbf{B} \\
\mathbf{B}\end{array}$ & $\begin{array}{l}8397.74 \\
8409.84 \\
8416.39 \\
8421.39 \\
8425.56\end{array}$ & $\begin{array}{l}1 \\
1 \\
1 \\
1 \\
1\end{array}$ & $\begin{array}{l}\mathbf{E} \\
\mathbf{E} \\
\mathbf{E} \\
\mathbf{E} \\
\mathbf{B}\end{array}$ & $\begin{array}{l}8677.93 \\
8706.32 \\
8708.43 \\
8717.89 \\
8724.55\end{array}$ & $\begin{array}{l}2 \\
2 \\
2 \\
3 \\
3 \\
1\end{array}$ & $\begin{array}{l}\mathrm{A} \\
\mathrm{B} \\
\mathrm{A} \\
\mathrm{A} \\
\mathrm{C}\end{array}$ \\
\hline $\begin{array}{l}8267.48 \\
8269.06 \\
8274.03 \\
8277.81 \\
8284.37\end{array}$ & $\begin{array}{l}1 \\
1 \\
1 \\
1 \\
1\end{array}$ & $\begin{array}{l}\mathbf{B} \\
\mathbf{D} \\
\mathbf{E} \\
\mathbf{E} \\
\mathbf{B}\end{array}$ & $\begin{array}{l}8427.40 \\
8432.64 \\
8437.67 \\
8439.76 \\
8454.77\end{array}$ & $\begin{array}{l}1 \\
3 \\
2 \\
1 \\
1\end{array}$ & $\begin{array}{l}\mathbf{B} \\
\mathbf{A} \\
\mathbf{B} \\
\mathbf{E} \\
\mathbf{A}\end{array}$ & $\begin{array}{l}8738.79 \\
8741.73 \\
8757.32 \\
8758.28 \\
8759.94\end{array}$ & $\begin{array}{l}1 \\
1 \\
1 \\
2 \\
1\end{array}$ & $\begin{array}{l}\mathrm{E} \\
\mathbf{B} \\
\mathrm{B} \\
\mathrm{A} \\
\mathrm{E}\end{array}$ \\
\hline $\begin{array}{l}8286.15 \\
8289.28 \\
8292.32 \\
8301.05 \\
8305.79\end{array}$ & $\begin{array}{l}1 \\
3 \\
1 \\
3 \\
4\end{array}$ & $\begin{array}{l}\text { C } \\
\text { B } \\
\mathbf{E} \\
\text { A } \\
\mathbf{A}\end{array}$ & $\begin{array}{l}8457.77 \\
8465.08 \\
8469.69 \\
8473.55 \\
8476.65\end{array}$ & $\begin{array}{l}2 \\
1 \\
1 \\
3 \\
1\end{array}$ & $\begin{array}{l}\mathrm{A} \\
\mathbf{B} \\
\mathrm{E} \\
\mathrm{A} \\
\mathrm{A}\end{array}$ & $\begin{array}{l}8768.61 \\
8780.59 \\
8785.78 \\
8788.83 \\
8808.40\end{array}$ & $\begin{array}{l}1 \\
1 \\
1 \\
2 \\
1\end{array}$ & $\begin{array}{l}\mathbf{E} \\
\mathbf{B} \\
\mathbf{E} \\
\mathbf{B} \\
\mathbf{B}\end{array}$ \\
\hline $\begin{array}{l}8308.99 \\
8312.76 \\
8315.45 \\
8318.90 \\
8320.12\end{array}$ & $\begin{array}{l}1 \\
2 \\
2 \\
1 \\
1\end{array}$ & $\begin{array}{l}\text { E } \\
B \\
B \\
B \\
B\end{array}$ & $\begin{array}{l}8478.24 \\
8480.09 \\
8486.00 \\
8498.33 \\
8506.78\end{array}$ & $\begin{array}{l}1 \\
1 \\
4 \\
1 \\
2\end{array}$ & $\begin{array}{l}\mathrm{C} \\
\mathrm{E} \\
\mathrm{A} \\
\mathrm{E} \\
\mathbf{A}\end{array}$ & $\begin{array}{l}8823.24 \\
8837.37 \\
8839.45 \\
8846.46 \\
8859.76\end{array}$ & $\begin{array}{l}1 \\
1 \\
1 \\
1 \\
2\end{array}$ & $\begin{array}{l}\mathbf{E} \\
\mathbf{E} \\
\mathbf{E} \\
\mathbf{E} \\
\mathbf{A}\end{array}$ \\
\hline $\begin{array}{l}8325.33 \\
8327.94 \\
8330.55 \\
8339.64 \\
8341.64\end{array}$ & $\begin{array}{l}1 \\
1 \\
1 \\
1 \\
1\end{array}$ & $\begin{array}{l}\mathbf{C} \\
\mathrm{E} \\
\mathrm{E} \\
\mathrm{E} \\
\mathrm{E}\end{array}$ & $\begin{array}{l}8510.92 \\
8521.21 \\
8522.86 \\
8523.87 \\
8539.22\end{array}$ & $\begin{array}{l}4 \\
1 \\
1 \\
1 \\
1 \quad b, d ?\end{array}$ & $\begin{array}{l}A \\
E \\
E \\
E \\
E \\
C\end{array}$ & $\begin{array}{l}8867.42 \\
8913.66 \\
8916.73 \\
8939.05 \\
8957.55\end{array}$ & $\begin{array}{l}1 \\
2 \\
1 \\
1 \\
1\end{array}$ & $\begin{array}{l}\mathbf{E} \\
\mathbf{A} \\
\mathbf{E} \\
\mathbf{E} \\
\mathbf{A}\end{array}$ \\
\hline $\begin{array}{l}8344.61 \\
8348.70 \\
8355.25 \\
8357.69 \\
8367.00\end{array}$ & $\begin{array}{l}1 \\
3 \\
2 \\
1 \\
1\end{array}$ & $\begin{array}{l}\mathbf{B} \\
\mathbf{A} \\
\mathbf{A} \\
\mathbf{E} \\
\mathbf{E}\end{array}$ & $\begin{array}{l}8543.22 \\
8557.12 \\
8568.94 \\
8575.80 \\
8577.24\end{array}$ & $\begin{array}{l}3 \\
1 \\
1 \\
1 \\
1\end{array}$ & $\begin{array}{l}\mathbf{B} \\
\mathbf{C} \\
\mathbf{B} \\
\mathbf{B} \\
\mathbf{E}\end{array}$ & $\begin{array}{l}8976.65 \\
8980.03 \\
8989.07 \\
9003.57 \\
9012.19\end{array}$ & $\begin{array}{l}1 \\
1 \\
1 \\
1 \\
1\end{array}$ & $\begin{array}{l}\mathbf{C} \\
\mathrm{E} \\
\mathbf{C} \\
\mathrm{E} \\
\mathrm{B}\end{array}$ \\
\hline $\begin{array}{l}8369.08 \\
8370.38 \\
8372.27 \\
8376.99 \\
8378.94\end{array}$ & $\begin{array}{l}1 \\
1 \\
2 \\
1 \\
1\end{array}$ & $\begin{array}{l}\mathrm{E} \\
\mathrm{E} \\
\mathrm{B} \\
\mathrm{E} \\
\mathrm{B}\end{array}$ & $\begin{array}{l}8586.05 \\
8600.44 \\
8609.29 \\
8612.03 \\
8617.05\end{array}$ & $\begin{array}{l}1 \\
1 \\
1 \\
1 \\
3\end{array}$ & $\begin{array}{l}\mathbf{B} \\
\mathbf{C} \\
\mathbf{C} \\
\mathbf{E} \\
\mathbf{A}\end{array}$ & $\begin{array}{l}9060.34 \\
9062.98 \\
9100.73 \\
9119.12 \\
9215.55\end{array}$ & $\begin{array}{l}1 \\
1 \\
1 \\
1 \\
1 \\
1\end{array}$ & $\begin{array}{l}\mathrm{C} \\
\mathrm{C} \\
\mathrm{E} \\
\mathrm{E} \\
\mathrm{E}\end{array}$ \\
\hline $\begin{array}{l}8383.71 \\
8387.77 \\
8390.29 \\
8393.65 \\
8394.92\end{array}$ & $\begin{array}{l}3 \\
3 \\
1 \\
2 \\
1\end{array}$ & $\begin{array}{l}\text { A } \\
\text { A } \\
\text { B } \\
\text { B } \\
\text { B }\end{array}$ & $\begin{array}{l}8622.27 \\
8632.83 \\
8641.46 \\
8645.02 \\
8666.16\end{array}$ & $\begin{array}{l}1 \\
3 \\
1 \\
1 \\
1\end{array}$ & $\begin{array}{l}\mathrm{A} \\
\mathrm{A} \\
\mathrm{E} \\
\mathrm{E} \\
\mathrm{C}\end{array}$ & & & \\
\hline
\end{tabular}

\section{LINES OF UNKNOWN ORIGIN}

Table 3 contains the wave lengths of lines that have been observed in the spectra of both neodymium and samarium. Later research may reveal them either as lines properly belonging to each spectrum and approximately of the same wave length or as lines belonging to the spectra of other known elements. In the meantime it is thought best to segregate them into a separate table so that if they are characteristic of a new element they may be of service to chemists and others interested in the isolation of the members of the rare earth elements. 
TABLE 3.-Lines of Unknown Origin Common to Nd and Sa

\begin{tabular}{|c|c|c|c|c|c|c|c|c|}
\hline$\lambda$ I. A. & Notes & $\begin{array}{c}\text { Probable } \\
\text { error }\end{array}$ & $\lambda$ I. A. & Notes & $\begin{array}{c}\text { Probable } \\
\text { error }\end{array}$ & $\lambda$ I. A. & Notes & $\begin{array}{c}\text { Probable } \\
\text { error }\end{array}$ \\
\hline $\begin{array}{l}5483.10 \\
508.41 \\
5510.08 \\
5513.17 \\
5523.88\end{array}$ & $\begin{array}{l}3 \\
3 \\
1 \\
2 \\
2\end{array}$ & $\begin{array}{l}\mathbf{A} \\
\text { A } \\
\text { B } \\
\text { A } \\
\mathbf{A}\end{array}$ & $\begin{array}{l}5714.74 \\
5716.06 \\
5720.83 \\
5739.97 \\
5740.86\end{array}$ & $\begin{array}{l}1 \\
1 \\
1 \\
3 \\
5\end{array}$ & $\begin{array}{l}\mathbf{B} \\
\mathbf{B} \\
\mathbf{B} \\
\mathbf{A} \\
\mathbf{A}\end{array}$ & $\begin{array}{l}6111.76 \\
6126.32 \\
6135.82 \\
6156.90 \\
6169.15\end{array}$ & $\begin{array}{l}1 \\
2 \\
2 \\
2 \\
2 \text { ES }\end{array}$ & $\begin{array}{l}\text { B } \\
\text { A } \\
\text { A } \\
\text { A } \\
\text { B }\end{array}$ \\
\hline $\begin{array}{l}5539.19 \\
5542.32 \\
5551.38 \\
5554.24 \\
5557.41\end{array}$ & $\begin{array}{l}2 \text { ES } \\
1 \\
3 \\
1 \\
2\end{array}$ & $\begin{array}{l}\mathbf{A} \\
\mathbf{A} \\
\mathbf{A} \\
\mathbf{B} \\
\mathbf{A}\end{array}$ & $\begin{array}{l}5758.88 \\
5765.46 \\
5775.49 \\
5789.88 \\
5792.40\end{array}$ & $\begin{array}{l}1 \\
2 \\
1 \\
2 \\
2\end{array}$ & $\begin{array}{l}\mathbf{A} \\
\mathbf{A} \\
\mathbf{B} \\
\mathbf{B} \\
\mathbf{B}\end{array}$ & $\begin{array}{l}6172.53 \\
6210.70 \\
6289.90 \\
6305.19 \\
6307.06\end{array}$ & $\begin{array}{l}2 \\
1 \\
2 \\
\frac{1}{2} \mathrm{Gd} ?\end{array}$ & $\begin{array}{l}\mathrm{A} \\
\mathrm{A} \\
\mathrm{A} \\
\mathrm{A} \\
\mathrm{A}\end{array}$ \\
\hline $\begin{array}{l}5558.10 \\
5562.78 \\
5563.77 \\
5566.62 \\
5576.42\end{array}$ & $\begin{array}{l}\frac{1}{2} \text { ES } \\
1 \\
1 \\
1 \\
1\end{array}$ & $\begin{array}{l}\mathbf{A} \\
\mathbf{A} \\
\mathbf{B} \\
\mathbf{B} \\
\mathbf{A}\end{array}$ & $\begin{array}{l}5796.43 \\
5903.10 \\
5807.81 \\
5818.30 \\
5822.63\end{array}$ & $\begin{array}{l}1 \\
1 \\
1 \\
2 \\
1\end{array}$ & $\begin{array}{l}\text { B } \\
\text { B } \\
\text { B } \\
\text { A } \\
\text { B }\end{array}$ & $\begin{array}{l}6308.28 \\
6317.72 \\
6330.14 \\
6346.53 \\
6394.91\end{array}$ & $\begin{array}{l}2 \\
1 \\
1 \\
1 \\
1\end{array}$ & $\begin{array}{l}\text { A } \\
\text { B } \\
\text { B } \\
\text { A } \\
\text { A. }\end{array}$ \\
\hline $\begin{array}{l}5583.25 \\
5585.09 \\
5585.61 \\
5596.96 \\
5597.57\end{array}$ & $\begin{array}{l}2 \\
1 \\
\frac{1}{1} \\
\frac{1}{2}\end{array}$ & $\begin{array}{l}\mathbf{A} \\
\mathbf{A} \\
\mathbf{A} \\
\mathbf{B} \\
\mathbf{B}\end{array}$ & $\begin{array}{l}5829.70 \\
5845.90 \\
5836.47 \\
5894.04 \\
5906.65\end{array}$ & $\begin{array}{l}3 \\
2 \\
2 \\
1 \\
2\end{array}$ & $\begin{array}{l}\mathbf{A} \\
\mathbf{A} \\
\mathbf{A} \\
\mathbf{B} \\
\mathbf{A}\end{array}$ & $\begin{array}{l}6454.83 \\
6461.20 \\
6506.32 \\
6520.33 \\
6637.17\end{array}$ & $\begin{array}{l}2 \\
1 \\
1 \\
1 \\
2\end{array}$ & $\begin{array}{l}\text { B } \\
\text { B } \\
\text { B } \\
\text { B } \\
\text { A }\end{array}$ \\
\hline $\begin{array}{l}5599.48 \\
5603.15 \\
5603.63 \\
5604.84 \\
5608.10\end{array}$ & $\begin{array}{l}1 \\
2 \\
3 \\
2 \\
1\end{array}$ & $\begin{array}{l}\mathbf{A} \\
\mathbf{A} \\
\mathbf{A} \\
\mathbf{A} \\
\mathbf{B}\end{array}$ & $\begin{array}{l}5913.86 \\
5927.26 \\
5932.48 \\
5935.54 \\
5949.11\end{array}$ & $\begin{array}{l}1 \\
1 \\
1 \\
1 \\
1\end{array}$ & $\begin{array}{l}\mathrm{B} \\
\mathbf{B} \\
\mathbf{B} \\
\mathbf{A} \\
\mathbf{B}\end{array}$ & $\begin{array}{l}6743.57 \\
6748.35 \\
6803.06 \\
6873.06 \\
6985.22\end{array}$ & $\begin{array}{l}1 \\
1 \\
3 \\
2 \\
1\end{array}$ & $\begin{array}{l}\text { B } \\
\text { B } \\
\text { A } \\
\text { A } \\
\text { B }\end{array}$ \\
\hline $\begin{array}{l}5608.42 \\
5615.68 \\
5616.88 \\
5617.67 \\
5623.59\end{array}$ & $\begin{array}{l}1 \text { ES } \\
1 \\
1 \\
3 \\
2\end{array}$ & $\begin{array}{l}\mathbf{B} \\
\mathbf{B} \\
\mathbf{A} \\
\mathbf{A} \\
\mathbf{A}\end{array}$ & $\begin{array}{l}5959.45 \\
5961.50 \\
5963.22 \\
5970.16 \\
5974.22\end{array}$ & $\begin{array}{l}1 \\
2 \\
2 \\
1 \\
2\end{array}$ & $\begin{array}{l}\mathbf{B} \\
\mathbf{A} \\
\mathbf{A} \\
\mathbf{B} \\
\mathbf{A}\end{array}$ & $\begin{array}{l}7018.87 \\
7095.51 \\
7101.44 \\
7227.19 \\
7254.95\end{array}$ & $\begin{array}{l}1 \\
2 \\
1 \\
1 \\
1 \mathrm{n} ?\end{array}$ & $\begin{array}{l}\mathbf{B} \\
\mathbf{A} \\
\mathbf{A} \\
\mathbf{B} \\
\mathbf{B} \\
\mathbf{B}\end{array}$ \\
\hline $\begin{array}{l}5630.10 \\
5635.03 \\
5641.60 \\
5650.80 \\
5651.95\end{array}$ & $\begin{array}{l}2 \\
1 \\
1 \\
1 \\
2\end{array}$ & $\begin{array}{l}\mathbf{A} \\
\mathbf{B} \\
\mathbf{B} \\
\mathbf{A} \\
\mathbf{A}\end{array}$ & $\begin{array}{l}5982.35 \\
5984.32 \\
5989.33 \\
6001.95 \\
6014.59\end{array}$ & $\begin{array}{l}2 \\
3 \\
3 \\
1 \\
2\end{array}$ & $\begin{array}{l}\mathbf{A} \\
\mathbf{A} \\
\mathbf{A} \\
\mathbf{B} \\
\mathbf{A}\end{array}$ & $\begin{array}{l}7794.53 \\
7798.37 \\
7823.31 \\
7868.14 \\
8026.32\end{array}$ & $\begin{array}{l}1 \\
2 \\
1 \\
1 \\
3\end{array}$ & $\begin{array}{l}\mathbf{A} \\
\mathbf{A} \\
\mathbf{A} \\
\mathbf{B} \\
\mathbf{A}\end{array}$ \\
\hline $\begin{array}{l}5657.87 \\
5681.92 \\
5685.97 \\
5692.60 \\
5694.54\end{array}$ & $\begin{array}{l}\frac{1}{2} \\
\frac{1}{2} \\
2 \mathrm{Cu} \mathrm{g} ?\end{array}$ & $\begin{array}{l}\mathbf{B} \\
\mathbf{A} \\
\mathbf{B} \\
\mathbf{A} \\
\mathbf{A}\end{array}$ & $\begin{array}{l}6030.33 \\
6053.83 \\
6061.85 \\
6074.43 \\
6075.70\end{array}$ & $\begin{array}{l}1 \\
3 \\
2 \\
1 \\
2\end{array}$ & $\begin{array}{l}\mathrm{A} \\
\mathrm{A} \\
\mathrm{A} \\
\mathrm{A} \\
\mathrm{A}\end{array}$ & $\begin{array}{l}8055.56 \\
8322.04 \\
8504.71 \\
8526.43 \\
8544.72\end{array}$ & $\begin{array}{l}1 \\
1 \\
2 \\
1 \\
1\end{array}$ & $\begin{array}{l}\mathbf{B} \\
\mathbf{B} \\
\mathbf{B} \\
\mathbf{B} \\
\mathbf{B}\end{array}$ \\
\hline $\begin{array}{l}5698.91 \\
5702.72 \\
5703.46 \\
5706.20 \\
5713.03\end{array}$ & $\begin{array}{l}3 \\
2 \\
2 \\
5 \\
1\end{array}$ & $\begin{array}{l}\mathbf{A} \\
\mathbf{A} \\
\mathbf{B} \\
\mathbf{A} \\
\mathbf{A}\end{array}$ & $\begin{array}{l}6084.11 \\
6039.65 \\
6095.68 \\
6098.20 \\
6104.35\end{array}$ & $\begin{array}{l}3 \\
1 \\
1 \\
1 \\
2\end{array}$ & $\begin{array}{l}\mathbf{A} \\
\mathbf{A} \\
\mathbf{B} \\
\mathbf{B} \\
\mathbf{B}\end{array}$ & . & & \\
\hline
\end{tabular}

WASHINGTON, January 6, I922. 


